San Jose State University

SJSU ScholarWorks

Master's Theses

Master's Theses and Graduate Research

Spring 2018

\title{
Preparation and Characterization of a Ferrocene Containing Main- Chain PEG-CNT Phase for Hybrid Supercapacitor Application
}

Shalaka Rahangdale

San Jose State University

Follow this and additional works at: https://scholarworks.sjsu.edu/etd_theses

\section{Recommended Citation}

Rahangdale, Shalaka, "Preparation and Characterization of a Ferrocene Containing Main-Chain PEG-CNT Phase for Hybrid Supercapacitor Application" (2018). Master's Theses. 4918.

DOI: https://doi.org/10.31979/etd.7y95-gp6r

https://scholarworks.sjsu.edu/etd_theses/4918

This Thesis is brought to you for free and open access by the Master's Theses and Graduate Research at SJSU ScholarWorks. It has been accepted for inclusion in Master's Theses by an authorized administrator of SJSU ScholarWorks. For more information, please contact scholarworks@sjsu.edu. 
PREPARATION AND THE CHARACTERIZATION OF A FERROCENE CONTAINING MAIN-CHAIN PEG-CNT PHASE FOR HYBRID SUPERCAPACITOR APPLICATION

\author{
A Thesis \\ Presented to \\ The Faculty of the Department of Chemistry \\ San José State University \\ In Partial Fulfillment \\ of the Requirements for the Degree \\ Master of Science
}

by

Shalaka Rahangdale

May 2018 
(C) 2018

Shalaka Rahangdale

ALL RIGHTS RESERVED 
The Designated Thesis Committee Approves the Thesis Titled

\author{
PREPARATION AND THE CHARACTERIZATION OF A FERROCENE \\ CONTAINING MAIN-CHAIN PEG-CNT PHASE FOR HYBRID \\ SUPERCAPACITOR APPLICATION \\ by \\ Shalaka Rahangdale
}

APPROVED FOR THE DEPARTMENT OF CHEMISTRY

SAN JOSÉ STATE UNIVERSITY

May 2018

$\begin{array}{ll}\text { Roger Terrill, Ph.D. } & \text { Department of Chemistry } \\ \text { Joseph Pesek, Ph.D. } & \text { Department of Chemistry } \\ \text { Chester Simocko, Ph.D. } & \text { Department of Chemistry }\end{array}$ 


\title{
ABSTRACT
}

\section{PREPARATION AND THE CHARACTERIZATION OF A FERROCENE CONTAINING MAIN-CHAIN PEG-CNT PHASE FOR HYBRID SUPERCAPACITOR APPLICATION}

\author{
by Shalaka Rahangdale
}

With the rapid development of the global economy, the depletion of fossil fuels, and increasing environmental pollution, there is an urgent need for new technologies associated with energy conversion and storage. Supercapacitors have attracted tremendous attention and are now extensively used for energy storage. They charge and discharge quickly for high power demands but have low energy density. The goal of the research was to synthesize electrode and electrolyte materials for the fabrication of a hybrid supercapacitor cell with high energy density. In this hybrid cell, carbon nanotubes were used as electrodes, which contribute to electrostatic capacitance, and redox polymer was used as an electrolyte which accounts for redox or faradaic capacitance, thus increasing the energy density. For the reduction half-cell, ferrocene dicarboxylic acid was polymerized with polyethylene glycol phase for an electrolyte. The electrolyte for oxidation half-cell was viologen-PEG polymer, prepared by a laboratory colleague, Rana Kanishka. The hybrid cell using these materials was fabricated in a sandwich model configuration. Further, electrochemical experiments were employed for assessing the performance of the cell. In conclusion, the electrode and electrolyte materials showed excellent electrochemical behavior and were appropriate for this type of hybrid cell. 


\section{ACKNOWLEDGMENTS}

I take this opportunity to express my sincere gratitude to Dr. Roger Terrill for accepting me into this group. His inspiring and encouraging way guided me to a deeper understanding of research and I could complete my work with full dedication and sincerity. I truly appreciate his patience and invaluable suggestions in writing the thesis. I am also highly indebted to Dr. Joseph Pesek and Dr. Chester Simocko for their time and participation as my committee members.

I also owe my loving thanks to my parents, my uncles, my aunts and my brothers for encouraging and providing me with the moral support needed during this project. I also dedicate this to my grandfather and my late grandmother. Without their love and blessings, I could not have completed this work. 


\section{TABLE OF CONTENTS}

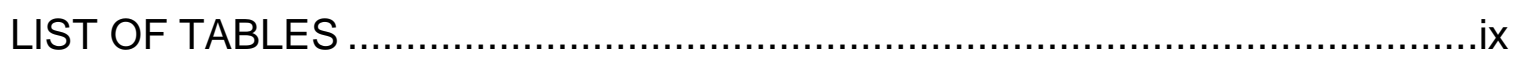

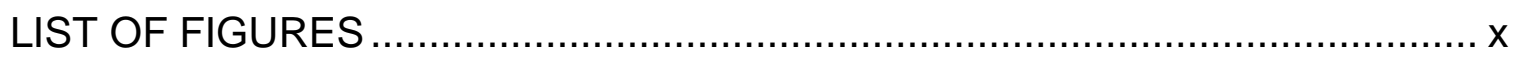

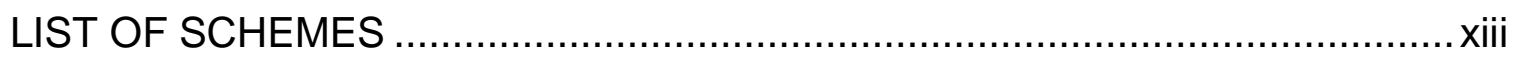

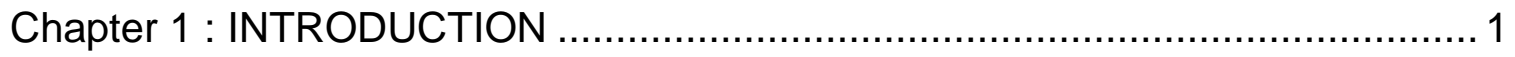

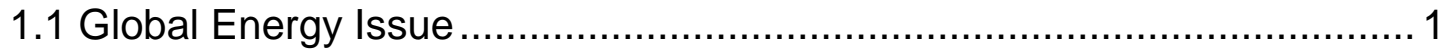

1.2 Energy Storage War................................................................. 2

1.3 Historical Background of Supercapacitors.......................................... 6

1.4 Energy Storage Mechanism of Supercapacitors ................................. 8

1.4.1 Electrochemical Double-Layer Capacitors .................................... 8

1.4.2 Redox-Based Supercapacitors ................................................. 12

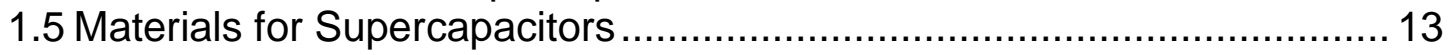

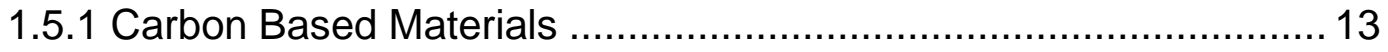

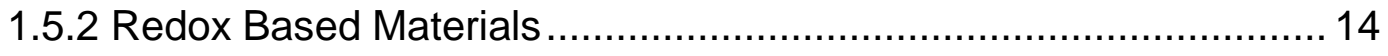

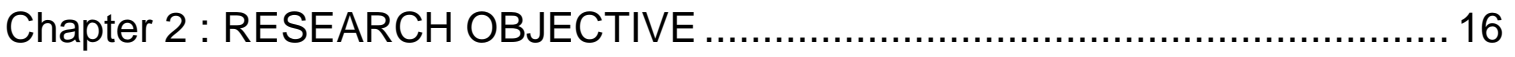

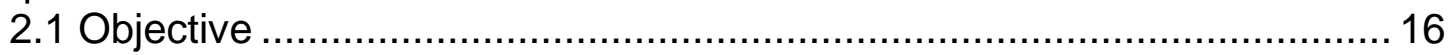

2.2 Electrode: Single Walled Carbon Nanotubes (SWCNTs) ....................... 17

2.3 Electrolyte: Polyethylene Glycol (PEG) - Ferrocene dicarboxylic acid -

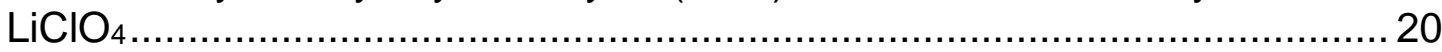

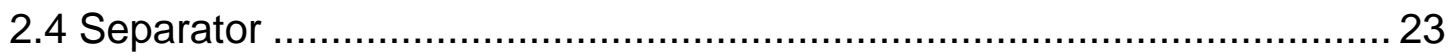

Chapter 3 : FUNCTIONALIZATION OF CARBON NANOTUBES …................. 25

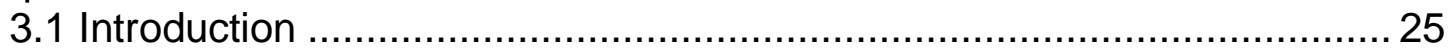

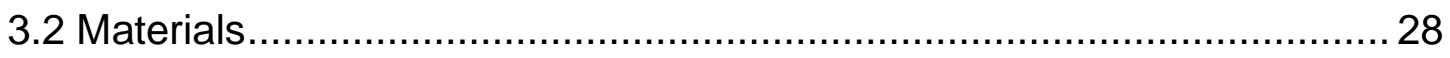

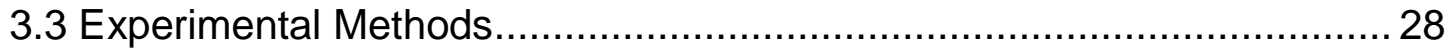

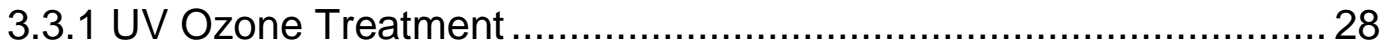

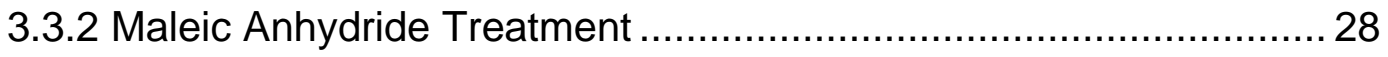

3.3.3 Concentrated Sulfuric/Nitric acid (3:1) Treatment ..........................29

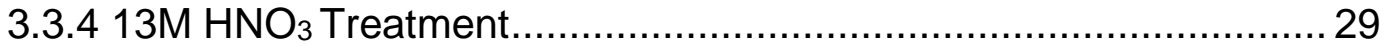

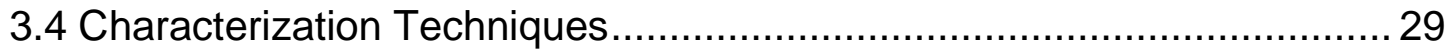

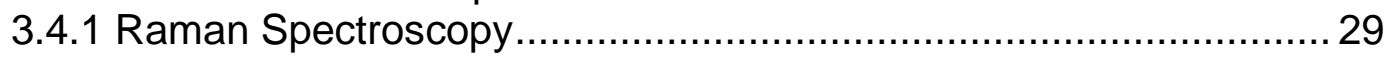

3.4.2 Fourier Transform Infrared Spectroscopy (FTIR) .......................... 32

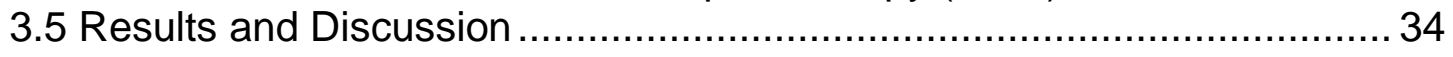

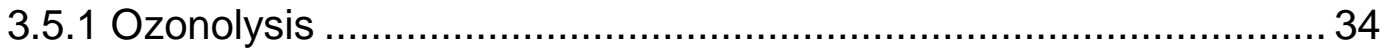

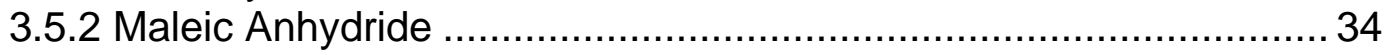

3.5.3 Concentrated Sulphuric/Nitric acid (3:1) .................................... 35

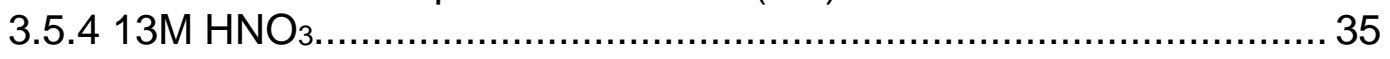




\section{Chapter 4 : SYNTHESIS OF POLYETHYLENE GLYCOL-FERROCENE} DICARBOXYLATE (FC-PEG) POLYMER …................................... 39

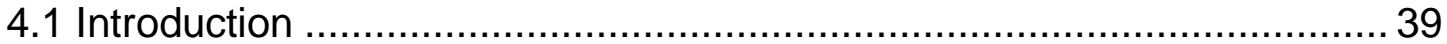

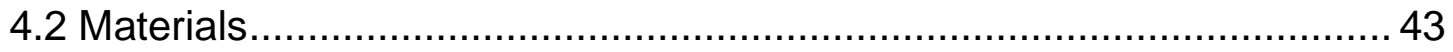

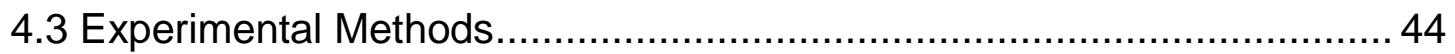

4.3.1 Acid Chloride Esterification ..................................................... 44

4.3.2 Steglish Esterification using Dicyclohexylcarbodiimide (DCC) ........ 45

4.3.3 Steglish Esterification using Diisopropylcardodiimide (DIC) ............ 45

4.4 Characterization Techniques..........................................................46

4.4.1 Nuclear Magnetic Resonance (NMR) ..................................... 46

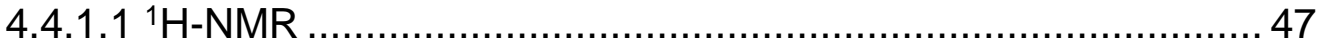

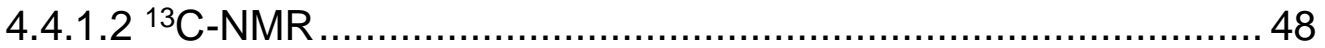

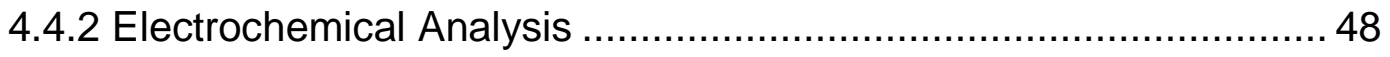

4.4.2.1 Solution Phase Electrochemistry ........................................ 51

4.4.2.2 Semi-Solid Phase Electrochemistry...................................... 53

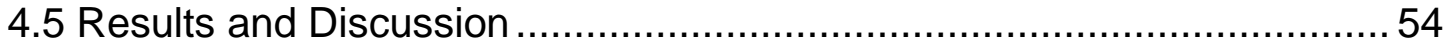

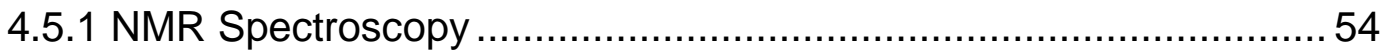

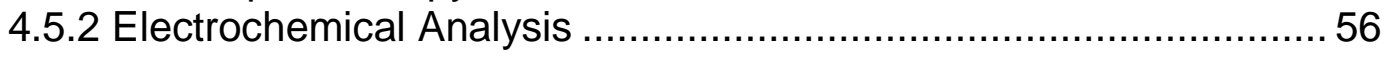

4.5.2.1 Solution Phase Electrochemistry ……................................... 56

4.5.2.2 Semi-Solid Phase Electrochemistry ..................................... 59

4.5.3 Fourier Transform Infrared Spectroscopy ..................................... 59

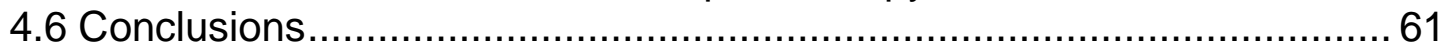

Chapter 5 : GRAFTING OF CARBON NANOTUBES WITH FERROCENE-

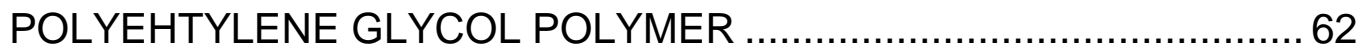

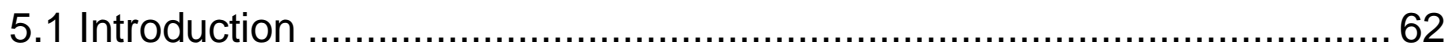

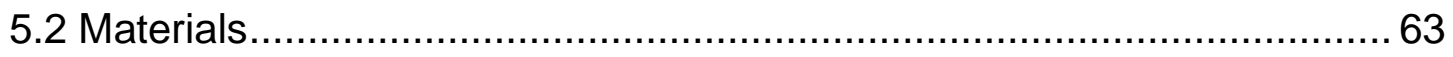

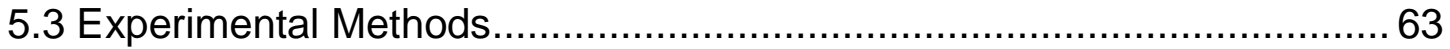

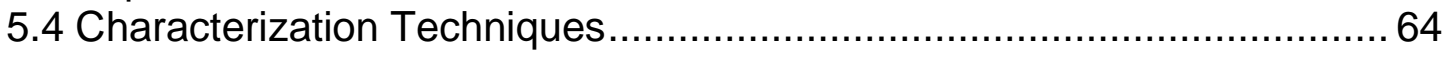

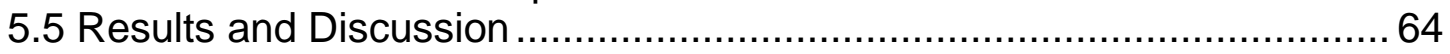

5.5.1 Fourier Transform Infrared Spectroscopy ..................................... 64

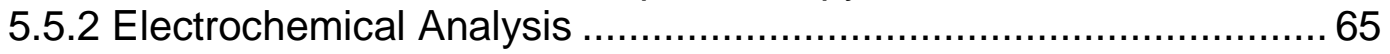

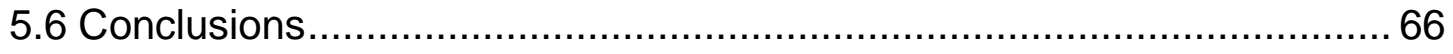

Chapter 6 : FABRICATION OF PROTOTYPE CELL ....................................... 68

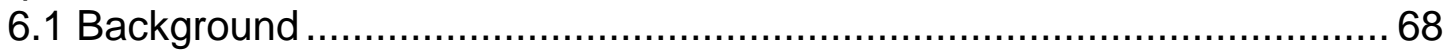

6.2. Cell Fabrication Procedure ........................................................... 72

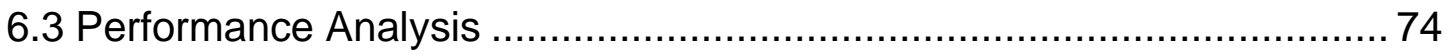

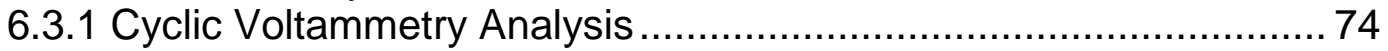

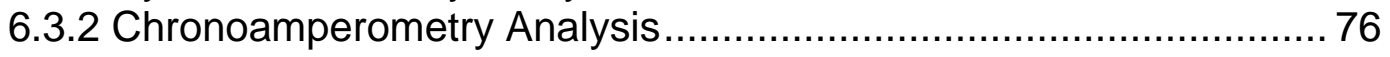

6.4 Conclusions and Future Work ……............................................... 79 


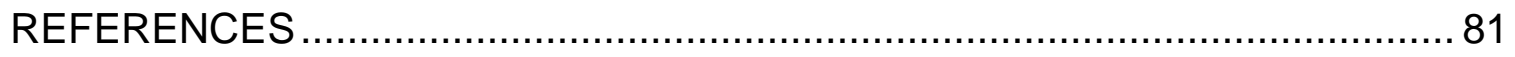




\section{LIST OF TABLES}

Table 1.1 Performance Comparison of Energy Storage Devices.......... 7

Table 6.1 Charging and discharging currents......................... 79 


\section{LIST OF FIGURES}

Figure 1.1 Schematic illustration of (a) charge and (b) discharge process of a lithium rechargeable battery

Figure 1.2 Ragone Plot of the energy storage domains for the various electrochemical energy conversion systems.

Figure 1.3 Schematic of electrochemical double-layer capacitor.......... 8

Figure 1.4 Model illustrating the double layer capacitance................ 10

Figure 1.5 The electrode resistance along with the Stern and diffuse layer capacitances in series.

Figure 2.1 Schematic of a portion of a graphene sheet rolled up to a SWCNT.

Figure 2.2 Ferrocene dicarboxylic acid - polyethylene glycol polymer...

Figure 2.3 Schematic electrolyte ionic accessibility in (a) liquid electrolyte and (b) polymer electrolyte

Figure 2.4 Lithium ion complexed in a polymer host............................. 22

Figure 2.5 Ferrocene dicarboxylic acid redox couple..................... 23

Figure $3.1 \quad$ Energy Level diagram.......................................... 31

Figure $3.2 \quad$ A multiple reflection ATR system........................... 33

Figure 3.3 Solubility test in water (1) pristine and (2) refluxed SWCNTs.

Figure 3.4 Raman spectra of (a) pristine and (b) refluxed SWCNTs...... 37

Figure 3.5 FTIR spectra of (a) pristine and (b) refluxed SWCNTs........ 38

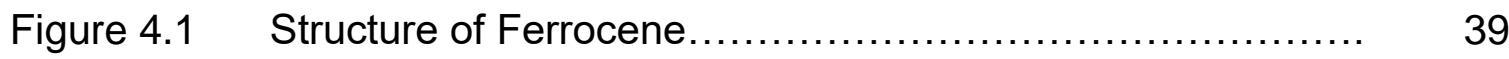

Figure $4.2 \quad$ Mechanism of the acid chloride formation.................... 41

Figure 4.3 Mechanism of the Steglish Esterification.............................. 42 
Figure 4.4 Different types of Fc-PEG polymer......................... 43

Figure 4.5 Experimental setup of liquid phase electrochemistry............ 52

Figure $4.6 \quad$ (a) Polymer deposited on microelectrode, (b) Experimental

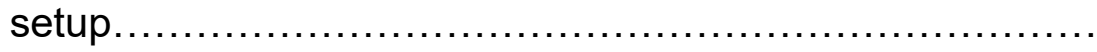

Figure 4.7 Experimental setup for semi-solid phase ultramicroelectrode voltammetry

Figure $4.8 \quad{ }^{1} \mathrm{H}-\mathrm{NMR}$ spectrum of Fc-PEG polymer......................... 55

Figure $4.9 \quad{ }^{13} \mathrm{C}-\mathrm{NMR}$ spectrum of FC-PEG polymer..................... 55

Figure 4.10 Cyclic Voltammogram of Ferrocene-dicarboxylic acid in acetonitrile with microelectrode.

Figure 4.11 Cyclic Voltammogram of Ferrocene-dicarboxylic acid in acetonitrile with ultramicroelectrode

Figure 4.12 Cyclic Voltammogram of Fc-PEG polymer with microelectrode.

Figure 4.13 Cyclic Voltammogram of Fc-PEG polymer with ultramicroelectrode.

Figure 4.14 Cyclic Voltammogram of Fc-PEG polymer using silver plate. 59

Figure 4.15 FTIR spectra of (a) Fc-PEG polymer, (b) ferrocenedicarboxylic acid, and (c) PEG

Figure 5.1 FTIR spectra of (a) SWCNT-Fc polymer and (b) refluxed SWCNTs. Inset (c) shows the FTIR spectrum of Fc-PEG polymer.

Figure 5.2 Cyclic Voltammogram of SWCNT-Fc polymer....................... 66

Figure 6.1 Mechanism of a hybrid supercapacitor cell during charging... 70

$\begin{array}{lll}\text { Figure 6.2 The chronoamperometric } & 71\end{array}$ experiment.

Figure 6.3 Aluminum current collectors with silicon gaskets and platinum foils. 
Figure 6.4 Aluminum current collectors with (a) analyte and (b)

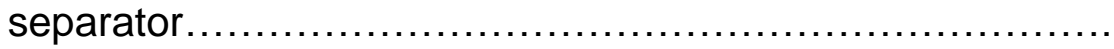

Figure 6.5 Configuration of the prototype cell in sandwich configuration..

Figure 6.6 Cyclic Voltammograms of the prototype cell at scan rates (a)

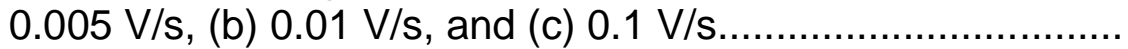

Figure 6.7 Chronoamperometry graphs of the prototype cell at (a) $0.5 \mathrm{~s}$,

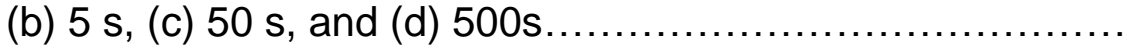




\section{LIST OF SCHEMES}

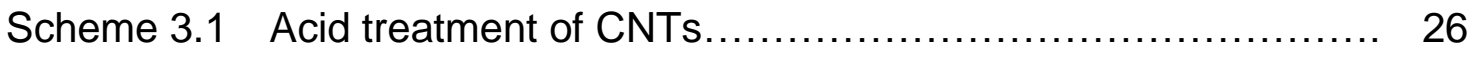

Scheme 3.2 The Diels Alder mechanism................................ 27

Scheme 4.1 Reaction scheme of the acid chloride esterification........... 43

Scheme 4.2 Reaction scheme of the Steglish esterification............... 43

Scheme 5.1 Scheme of (a) acyl chlorination of SWCNTs and (b) formation of SWCNT-Fc polymer composite..................... 62 


\section{Chapter 1 : INTRODUCTION}

\subsection{Global Energy Issue}

The topic of energy is a critical technological issue in the $21^{\text {st }}$ century. With the rapid development of the global economy, leading to an increase in energy demands, the cost of fossil fuels is increasing rapidly. This unchecked use of fossil fuels has increased pollution, leading to global warming which is creating a crisis in the modern society. ${ }^{1}$ To mitigate these issues, there is an urgent need to develop new energy conversion and storage technologies. There has been a growing interest in high energy and power density storage systems which can be used to store energy from renewable sources. These systems can achieve longterm clean energy solutions capable of meeting the ever-increasing needs of the world population. ${ }^{2,3}$

Conventional methods for energy conversion such as combustion used in heat-engine based power plants are pressure-volume processes which result first in mechanical and then in electrical energy. However, electrochemical technology, including batteries, fuel cells and supercapacitors, is based on interfacial energy or charge transfer. The ideal Carnot efficiency of an electrochemical cell, for example, a fuel cell, is about $94 \%$, which is much higher than the efficiency of a heat engine (40 to $60 \%))^{4,5}$

Thus, electrical energy storage is the key to increasing the efficiency of transportation systems and could replace the powertrains of current transportation systems from chemical fuel-based into an electrical one. Among 
these storage devices, supercapacitors are gaining attention because of their high-power density, long shelf life, fast charging/discharging rates, and simple operation.

\subsection{Energy Storage Market}

Supercapacitors offer a promising approach to meeting the increasing twentyfirst century power demands of energy storage systems as they have more than a thousand times the power density of lithium ion batteries and more than a hundred times the energy density of conventional capacitors. Supercapacitors can be used to increase the efficiency of hybrid electric vehicles by using the regenerative braking principle, in which energy is stored when the vehicle slows down or stops. ${ }^{6,7}$ For example, supercapacitors from Maxwell Technologies are being commercially used in Chinese hybrid buses. Supercapacitors are also being used in consumer electronics, memory back-up systems, industrial power, and energy management. ${ }^{8}$

There are two types of batteries, primary (non-rechargeable batteries) and secondary (rechargeable batteries). Primary batteries include alkaline, mercury, silver oxide, and zinc carbon batteries. Secondary batteries include lead acid batteries, nickel-cadmium (Ni-Cd), nickel-metal hydride ( $\mathrm{NiMH})$, and lithium ion batteries. Currently, the dominant energy storage devices are secondary lithiumion batteries. Rechargeable batteries such as nickel-cadmium and nickel-metal hydride cells are fading in popularity because of the performance degradation that they experience at low temperatures and high discharge rates. Lithium ion 
batteries have replaced other rechargeable batteries due to their higher energy density and lower weight. ${ }^{9}$ Worldwide, nearly every portable electronic device and electric vehicle (e.g. Tesla, Chevy Volt) is powered by lithium ion batteries.

Batteries store energy by converting chemical energy into electrical energy via redox reactions at the anode and cathode. ${ }^{9}$ In lithium ion batteries, the movement of lithium ions stores energy as illustrated in Figure 1.1. ${ }^{10}$ In these batteries, the intercalation/de-intercalation cycle of Li-ions between two layered compounds stores the electrochemical energy. ${ }^{11}$ During charging, the lithium ions flow from cathode to anode through the electrolyte. Correspondingly, electrons flow from cathode to anode via the external circuit. The electrons and ions combine at the anode and deposit the lithium there. The battery is fully charged and ready to use when no more ions flow reversibly. During discharging, the ions flow back to cathode through the electrolyte and electrons flow back via the external circuit, powering the electronic device. The ions and electrons combine at the cathode. When all the lithium ions move back to the cathode, the battery is fully discharged. 


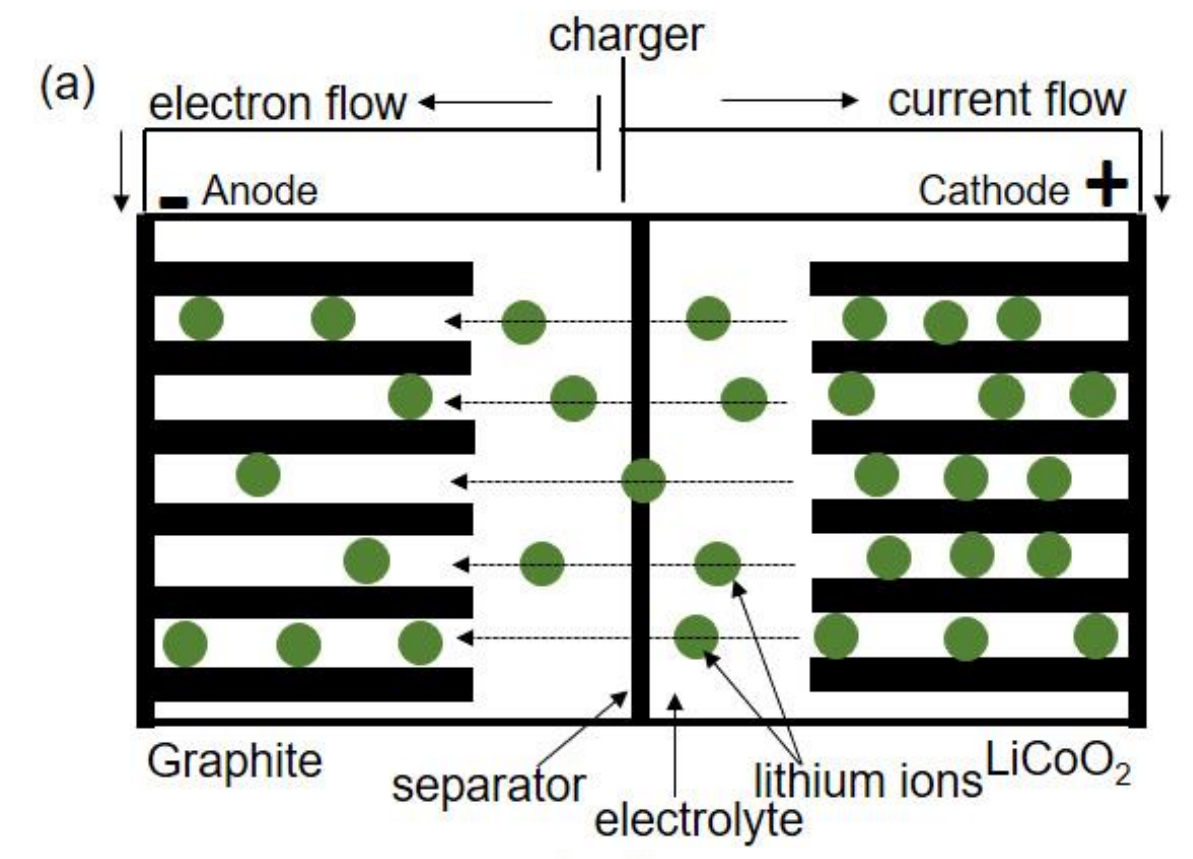

(b)

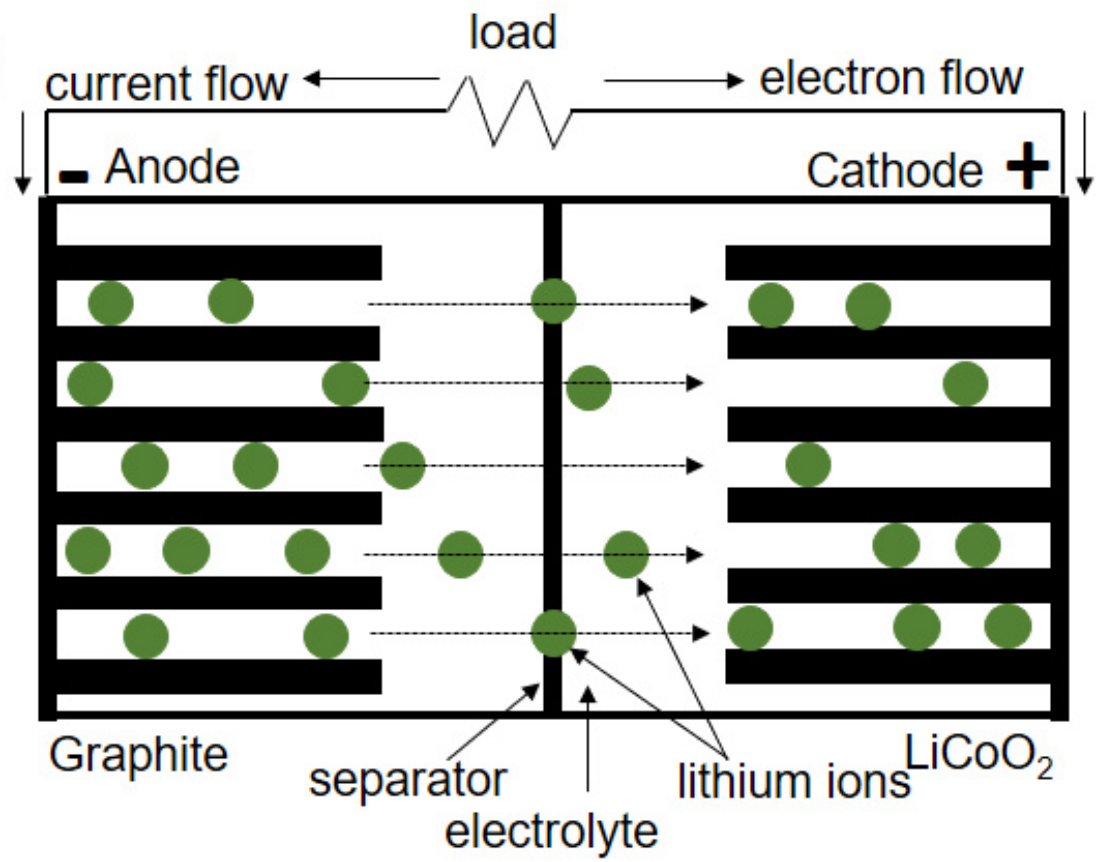

Figure 1.1 Schematic illustration of (a) charge and (b) discharge process of a lithium rechargeable battery.

The lithium ion battery has an energy density of $150-190 \mathrm{Wh} / \mathrm{kg}$ which is much higher than other rechargeable batteries, including lead-acid (30-50 $\mathrm{Wh} / \mathrm{kg})$, Ni-Cd (45-80 Wh/kg), and NiMH (60-120 Wh/kg). The Li-ion battery has 
longer shelf life when not in use as it self-discharges more slowly than other batteries. It is also quicker to charge and can handle thousands of chargedischarge cycles. However, improvement of the lithium ion battery is crucial for improving the technological infrastructure. Chemical degradation inside the lithium ion battery results in a slowing of the charge and discharge process, and diminished charge retention, thus reducing power density. Finally, such energy dense batteries require protection from being charged and discharged too quickly. High charge/discharge rates can cause overheating and tend to degrade the battery components. ${ }^{7}$

A relatively new class of energy storage devices, known as super- or ultracapacitors, can store a large amount of charge, deliver it at high power densities, and has a longer shelf life than batteries. ${ }^{12}$ The performance comparison of various energy storage devices is shown in the Ragone plot in Figure 1.2. ${ }^{9}$ This plot graphically represents the power density, measured along the vertical axis versus the energy density, measured along the horizontal axis. It can be seen that the supercapacitor performance lies between that of batteries and that of conventional capacitors. ${ }^{13}$ The energy density of supercapacitors is much higher than conventional capacitors, but still lower than batteries. Commercially available supercapacitors have specific energy below $10 \mathrm{Wh} / \mathrm{kg}$, which is 3-15 times lower than batteries (Li-ion batteries have $150 \mathrm{Wh} / \mathrm{kg}$ specific energy). ${ }^{14} \mathrm{As}$ a result, there is an increase in research interest to enhance the energy performance of a supercapacitor as compared to a battery. 


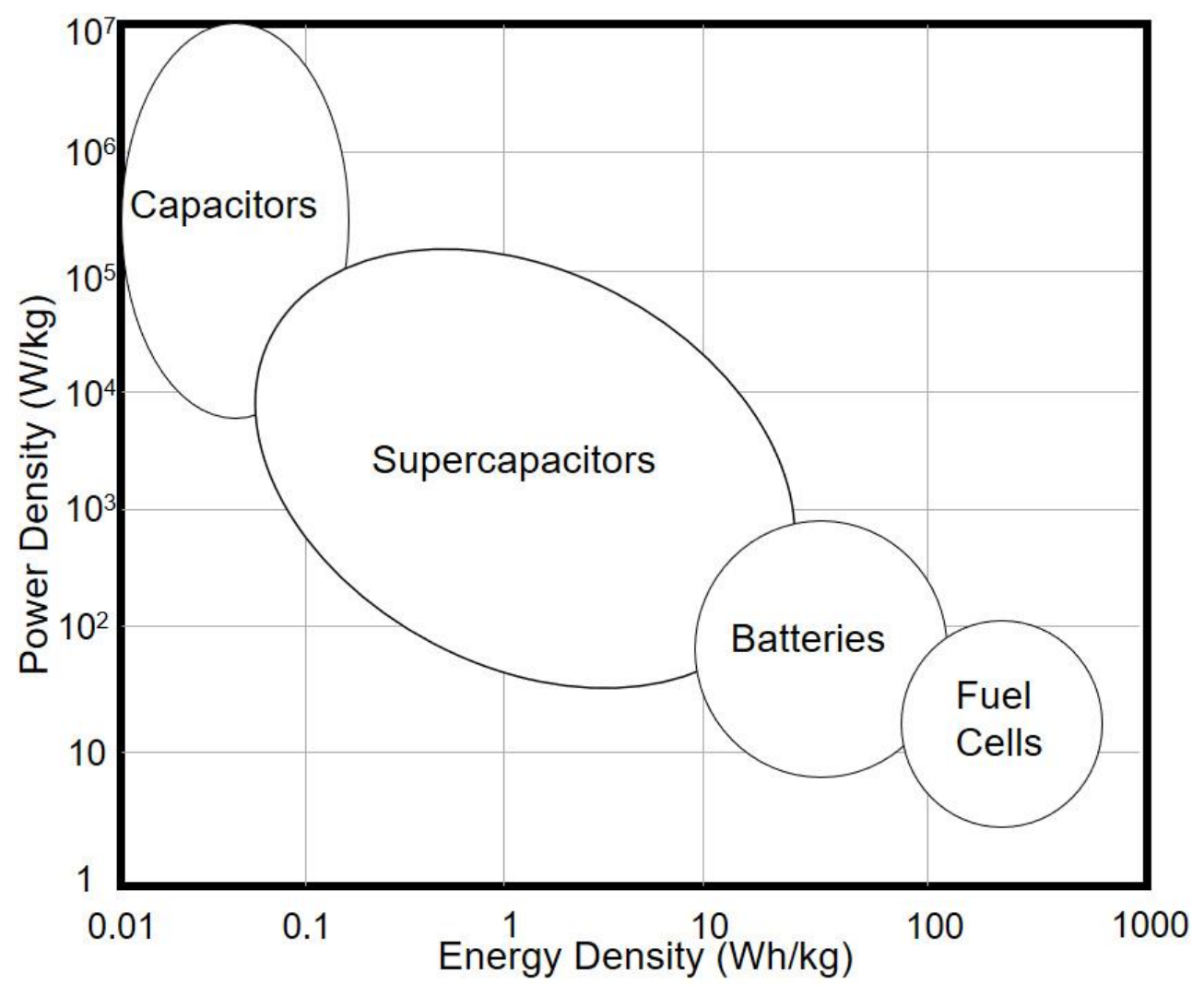

Figure 1.2 Ragone Plot of the energy storage domains for the various electrochemical energy conversion systems. ${ }^{9}$ Adapted with permission from Winter, M.; Brodd, R. J. What are Batteries, Fuel Cells, and Supercapacitors? Chem. Rev. 2004, 104, 4245-4269. Copyright 2004 American Chemical Society.

\subsection{Historical Background of Supercapacitors}

A supercapacitor is an electrochemical device which stores energy via electrostatic charges on the opposite surfaces of the electric double layer which is formed between the electrode and electrolyte. ${ }^{15}$ The first proposed supercapacitor was based on a porous carbon material with high surface area and a patent was granted to Becker and General Electric Corporation in 1957. In 1971, the Nippon Electric Company (NEC) produced low-power devices for memory backup applications. The Matsushita (Panasonic) released the 'Gold Capacitor' in 1978 and by 1987 ELNA produced a similar, low-power device 
called the 'Dynacap'. The Pinnacle Research Institute (PRI) developed the first high-power double-layer capacitor for military applications in $1982 .{ }^{16}$

Electrochemical double layer capacitors are now commercially available from a range of sources, and all are based on either a high surface area porous carbon material or noble metal oxides. The Maxwell Technologies, AVX, and Cooper Electronic Technologies in the United States, ELNA and Matsushita in Japan, ESMA in Russia, and Cap-XX in Australia all sell various types of double layer capacitor devices. ${ }^{16}$ The performance comparison of a capacitor, battery, and supercapacitor is given in Table 1.1.6,9 Supercapacitors are extremely efficient and can withstand a large number of charge/discharge cycles. They use low cost and environmentally friendly materials, they can store or release energy very quickly, and they lose energy heat in very small amounts. Additionally, supercapacitors do not contain toxic materials and are safer than batteries.

Table 1.1 Performance Comparison of Energy Storage Devices.

\begin{tabular}{|l|l|l|l|}
\hline Characteristics & Capacitor & Battery & Supercapacitor \\
\hline Specific energy $\left(\mathrm{W} \mathrm{h} \mathrm{kg}^{-1}\right)$ & $<0.1$ & $10-100$ & $0.1-10$ \\
\hline Specific power $\left(\mathrm{W} \mathrm{kg}^{-1}\right)$ & $>100,000$ & $10-1000$ & $50-100,000$ \\
\hline Discharge time & $10^{-6}$ to $10^{-3} \mathrm{~s}$ & $0.3-3 \mathrm{~h}$ & s to min \\
\hline Charge time & $10^{-6}$ to $10^{-3} \mathrm{~s}$ & $1-5 \mathrm{~h}$ & $\mathrm{~s}$ to min \\
\hline Efficiency $(\%)$ & About 100 & $70-85$ & $85-98$ \\
\hline Cycle-life & $\begin{array}{l}\text { Almost } \\
\text { infinite }\end{array}$ & About 1000 & $>500,000$ \\
\hline
\end{tabular}




\subsection{Energy Storage Mechanism of Supercapacitors}

Supercapacitors can be of two types depending on their energy storage mechanisms namely, electrochemical double-layer capacitors and redox based supercapacitors.

\subsubsection{Electrochemical Double-Layer Capacitors}

The electrochemical double-layer capacitors (EDLCs) store charge electrostatically (i.e. non-Faradaically), and thus are more like conventional electrolytic capacitors. An EDLC consists of two carbon-based electrodes, an electrolyte, and a separator. A defining characteristic of the EDLC is that there is no transfer of charge between electrodes and the electrolyte. The schematic illustration of EDLC is shown in Figure 1.3. ${ }^{17}$

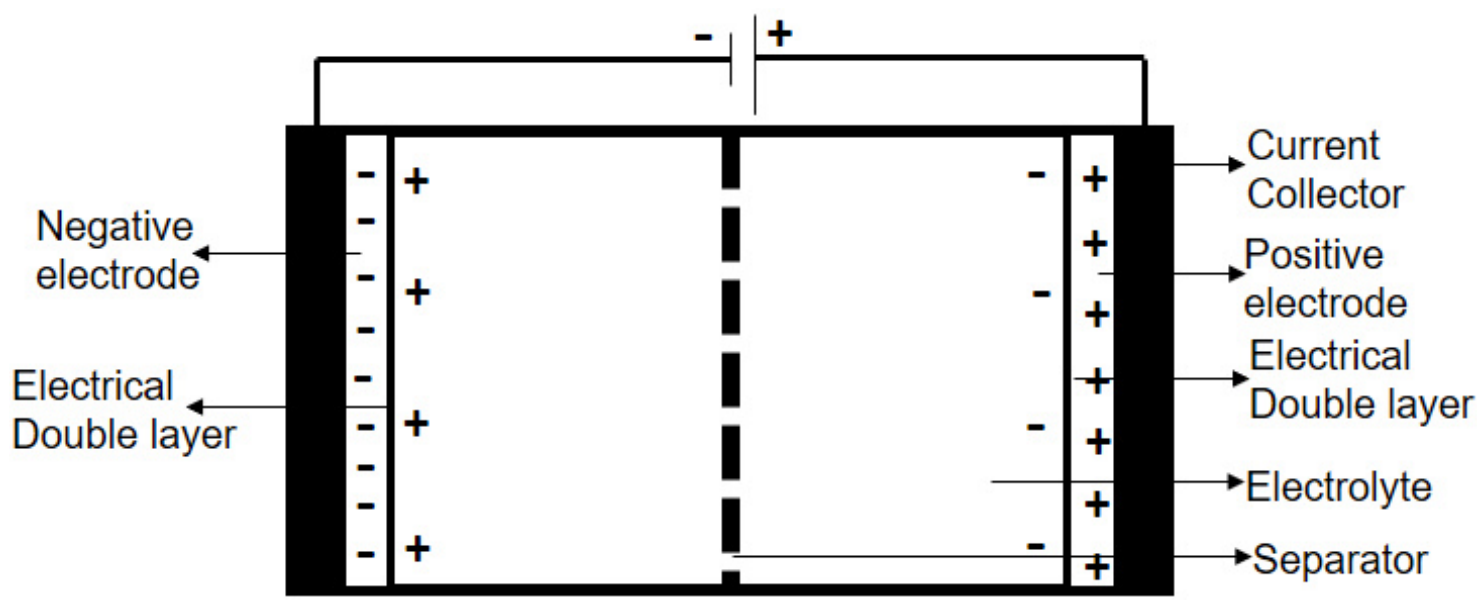

Figure 1.3 Schematic of electrochemical double-layer capacitor.

In an EDLC, charge is stored electrostatically using reversible adsorption of electrolyte ions onto electrochemically stable active materials which have high specific surface area. ${ }^{18}$ When voltage is applied, charge accumulates on the electrode surfaces and the oppositely charged electrolyte ions diffuse into the 
pores of the electrodes. In an EDLC the porous carbon creates an enormous surface area, which, in contact with an electrolyte has a substantial interfacial capacitance. These properties allow EDLCs to achieve higher energy densities than conventional capacitors. ${ }^{12,17,19}$ Each electrode/electrolyte interface contributes to the capacitance and each represents a capacitor, thus the EDLC is equivalent to two capacitors in a series. During charging, the cations move towards the negative electrode and anions move towards the positive electrode within the electrolyte. The electrons travel from the negative electrode to the positive electrode via the external circuit. This electrochemical process for charging is expressed as follows. At positive electrode:

$$
E_{1}+A^{-} \rightarrow E_{1}^{+} / / A^{-}+e^{-}
$$

$E_{1}$ is the positive electrode, $A^{-}$is the anion, and $/ /$represents the interface of electrode and electrolyte. At the negative electrode:

$$
\mathrm{E}_{2}+\mathrm{C}^{+}+\mathrm{e}^{-} \rightarrow \mathrm{E}_{2}^{-} / / \mathrm{C}^{+}
$$

$\mathrm{E}_{2}$ is the negative electrode and $\mathrm{C}^{+}$is the cation. During discharging, the reverse processes occur.

The most commonly used model to explain the principle of the double layer capacitance was put forth by Helmholtz in 1853 and is illustrated in Figure 1.4. ${ }^{15,20}$ This model consists of three planes: Inner Helmholtz plane (IHP), Outer Helmholtz plane (OHP), and Diffuse Layer. ${ }^{21}$ The IHP comprises of the dielectric medium formed by the monolayer of the solvent molecules of an electrolyte between the opposite charges. It has the thickness of a single molecule. This 
layer is formed when the dipoles in the solvent molecules interact with the charged electrode surface and orient themselves with the oppositely charged end near an electrode surface. There are also partially solvated, specifically adsorbed ions of the electrolyte along this layer. The OHP is the second layer at the electrode-electrolyte interface. Along the Helmholtz plane there is a linear variation of the potential with distance and comprises an excess of solvated ions of the complementary charge to that of the electrode. Lastly, the diffuse layer forms for a few nanometers into the solution where the variation of the potential becomes approximately exponential. ${ }^{22}$

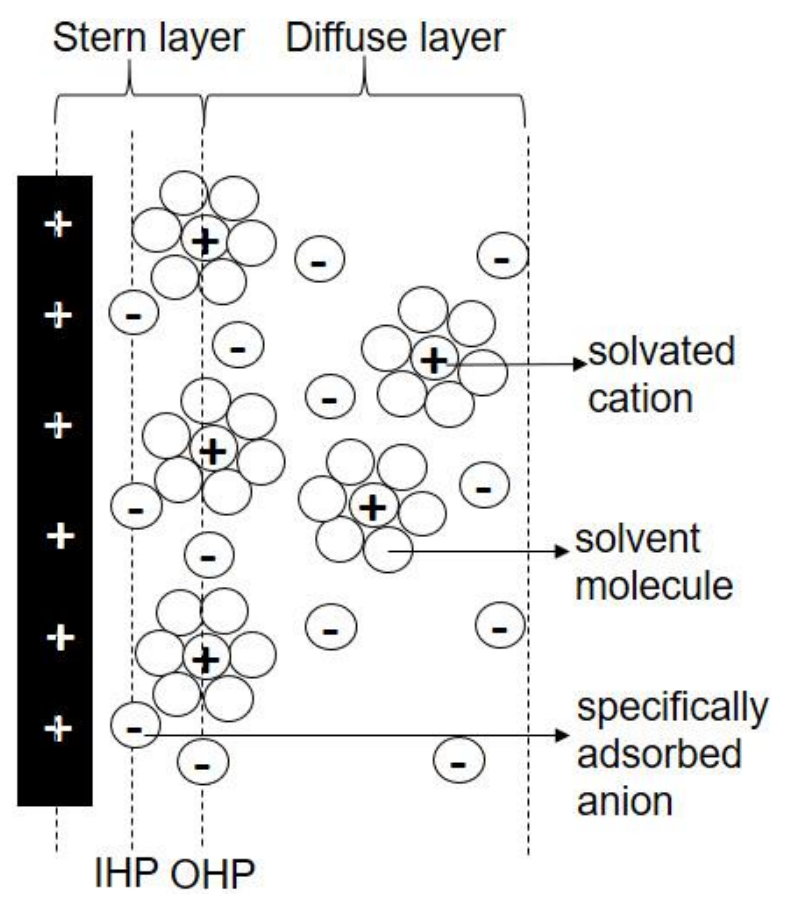

Figure 1.4 Model illustrating the double layer capacitance.

The electrical representation of an EDLC is shown in Figure $1.5^{22}$, where the electrolyte resistance is in series with the Stern layer and diffuse layer capacitances. ${ }^{22}$ The overall capacitance of an EDLC is given as follows: 


$$
\frac{1}{C}=\frac{1}{C_{s}}+\frac{1}{C_{d}}
$$

where $C_{s}$ is the capacitance of the Stern layer and $C_{D}$ is the capacitance of the diffuse layer. For any capacitor, the specific capacitance is:

$$
C=\frac{\varepsilon_{\mathrm{r}} \varepsilon_{0} A}{\mathrm{~d}}
$$

Equation 1.4

where $\varepsilon_{r}$ (dimensionless constant) is the electrolyte dielectric constant, $\varepsilon_{0}\left(\mathrm{Fm}^{-1}\right)$ is the permittivity of a vacuum, $\mathrm{A}\left(\mathrm{m}^{2} \mathrm{~g}^{-1}\right)$ is the specific area of the electrode accessible to the electrolyte ions, and $d(m)$ is the effective thickness of the double layer. ${ }^{23}$ The energy density $E$ and the power density $P$ of an electrochemical supercapacitor is expressed as:

$$
\begin{gathered}
E=\frac{1}{2} C V^{2} \\
P=\frac{V^{2}}{4 R_{s}}
\end{gathered}
$$

where $C$ is the specific capacitance, $V$ is the voltage applied on cell, and $R_{s}$ is the equivalent series resistance (ESR). ESR is the resistance contributed by the internal components of the capacitor like current collectors, electrodes, and dielectric material. ${ }^{17}$

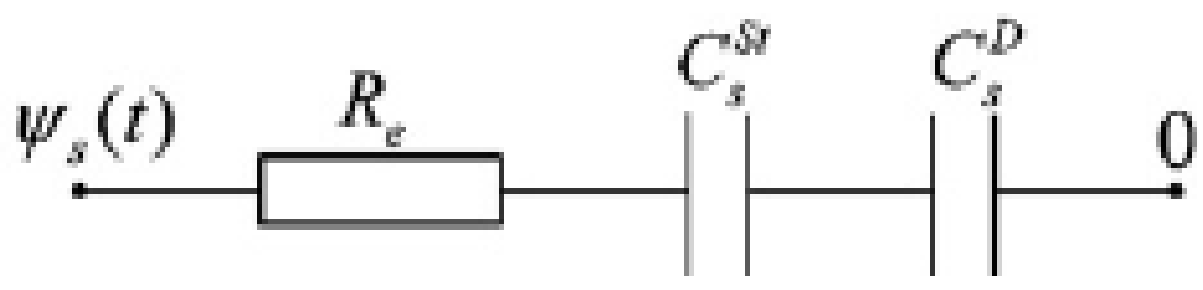

Figure 1.5 The electrode resistance along with the Stern and the diffuse layer capacitances in series. 
In EDLCs, generally the carbon electrode material with the higher surface area is used. As the surface area is increased, the capacitance is increased (from equation 1.4). In summary, EDLCs have higher capacitance as compared to conventional capacitors. Different carbon materials that can be used to store charge in EDLC electrodes are activated carbon, carbon aerogels, and carbon nanotubes.

\subsubsection{Redox-Based Supercapacitors}

The redox capacitor is an electrochemical capacitor which stores charge via faradaic process, i.e., reduction and oxidation reactions of the electrode material. ${ }^{12}$ Like an EDLC, it also consists of two electrodes separated by a separator and an electrolyte. ${ }^{17}$

The faradaic process involves transfer of charge by means of redox reactions. When an external potential is applied to a redox capacitor, a fast and reversible redox reaction takes place on the electrode. The reactions do not propagate into the bulk material and occurs at the electrode/electrolyte interface. ${ }^{12}$ The mechanisms of charge and discharge is similar to that of a battery. The theoretical redox capacitance of metal oxide can be calculated as:

$$
C=\frac{n \times F}{M \times V}
$$

Equation 1.7

where $\mathrm{n}$ is the mean number of electrons transferred in the redox reaction, $\mathrm{F}$ is the Faraday constant, $\mathrm{M}$ is the molar mass of metal oxide, and $\mathrm{V}$ is the operating voltage window. ${ }^{12}$ In redox capacitors, two types of electrode materials are used 
to store charge: conducting polymers and metal oxides. ${ }^{17}$ The redox reaction occur in the electroactive material.

\subsection{Materials for Supercapacitors}

The selection of an electrode material is critical in determining the electrochemical performance of the supercapacitor. The surface characteristics of the electrode greatly affect the capacitance of the cell as capacitive charge storage is a surface process. The carbon-based materials are the most widely used electrode materials in the EDLC, for their high specific surface area, high specific capacitance, good conductivity, and high chemical stability. The redox based materials are used in combination with double layer materials for their promising electrochemical activity.

\subsubsection{Carbon Based Materials}

Carbon-based materials, from activated carbons (ACs) to carbon nanotubes (CNTs) are used in batteries and supercapacitors because of their desirable physical and chemical properties. These properties include ease of processing, relative electrochemical inertness, low cost, wide temperature range, controllable porosity, and electro-catalytic activity for a variety of redox reactions. To ensure a good performance of the supercapacitor in terms of both energy and power density, requires proper control over the specific surface area and the effective pore size and matching to an appropriate type of electrolyte solution. ${ }^{23}$ Different types of carbon based materials used as electrodes are AC, CNTs, graphene, carbon aerogel, template carbons, and carbon-based composites. 
AC has a relatively high specific capacitance compared to other carbon materials, but has lower conductivity, whereas CNTs and graphene have low SC and higher conductivity. Recent studies have indicated that doping with heteroatoms such as $\mathrm{N}, \mathrm{O}, \mathrm{S}$, and $\mathrm{B}$ may improve capacitive performance, electrical conductivity, and wettability of the carbon phases. ${ }^{24}$

\subsubsection{Redox Based Materials}

The supercapacitor based on redox-active materials are highly desirable as the next generation electrochemical supercapacitor because they have an effective capacitance 10-100 times greater than the EDLC. They not only store charge in the double layer, but also undergo fast and reversible redox reactions. Hence, efforts have been made to develop electrode materials with intrinsic redox capacitance. The materials used are classified into two types: metal oxides and conducting polymers.

Generally, the metal oxides provide higher energy density for supercapacitors than conventional carbon materials. The general requirements for metal oxide in supercapacitor applications are: the oxide should be electronically conductive; the metal centers should have two or more accessible oxidation states; and protons should freely intercalate into the oxide lattice upon reduction. ${ }^{25}$ Transition metal oxides are considered the best electrode material for redox supercapacitors because they possess a variety of available oxidation states. ${ }^{20}$ The most investigated metal oxides are ruthenium oxide, manganese oxide, cobalt oxide, nickel oxide, and vanadium oxide. 
Conducting polymers (CPs) have many properties that make them suitable material for supercapacitor, such as low cost, environmental stability, high voltage capability, high redox storage capacity, and an adjustable redox activity through chemical modification. The redox capacitance of CP occurs through the reversible oxidation and reduction of the conjugated double bonds in a polymer network. During oxidation, ions are transferred to the polymer backbone, and during reduction, ions are released from this backbone into the electrolyte. The most extensively studied conducting polymers are polyaniline (PANI) and polypyrrole (PPy). ${ }^{17,20,25}$

Research is being carried out in developing materials for supercapacitors to increase energy and power density. Redox based materials, such as conducting polymers PANI and PPy, have gained tremendous attention. These materials are promising in combination with nanostructured carbon and metal oxides. Such composites have shown enhanced energy and power densities, and a good cycle life. ${ }^{25}$ 


\section{Chapter 2 : RESEARCH OBJECTIVE}

\subsection{Objective}

The motivation for this research work is to fabricate a hybrid supercapacitor cell. The hybrid supercapacitor combines a battery and an electric double-layer capacitor. It utilizes both faradaic (electrochemical charge transfer) and nonfaradaic (electrostatic charge storage) processes to store energy. The combination of redox and double-layer capacitance modes of energy storage should result in an increased energy and power density to fulfill the growing demand of applications. ${ }^{17}$ In general, research has been devoted to develop electrode materials with high capacitance and electrolytes with wide potential windows to increase the energy density of the two charge storage modes.

It is especially important to develop an electrolyte with a wide potential

window because the capacitive energy density $\left(E=\frac{1}{2} C V^{2}\right)$ is proportional to the square of the cell voltage. Thus, it is more efficient to increase the cell voltage than to increase the electrode capacitance to improve energy density. In this endeavor, the interaction between the electrolyte and the electrode material plays a crucial role. ${ }^{26}$

The hybrid approach in this work is facilitated by an incorporation of the redox-active charge storage species within the polymer electrolyte backbone. The redox species, ferrocene-dicarboxylic acid, is prepared as a copolymer with polyethylene glycol (PEG, 400 molecular weight). In this way, a substantial 
concentration of a redox-active ferrocene can be included in the polymer phase without concern for its solubility in either of the $\mathrm{Fe}^{2+}$ or $\mathrm{Fe}^{3+}$ forms.

In this work, a reduction half-cell was fabricated using the redox-PEG copolymer containing ferrocene dicarboxylic acid with carbon nanotubes as an electrode. The oxidation half-cell can be constructed with a redox species capable of undergoing electrochemical reduction in a potential range significantly negative, like viologen polymer species, detailed in the companion thesis by Kanishka Rana ${ }^{27}$. The redox behavior of this cell was studied by cyclic voltammetry and chronoamperometry. The energy density of this type of hybrid battery is expected to increase due to the combination of both redox and doublelayer capacitances. The materials used for the fabrication of the cell and their significance are discussed in later sections.

\subsection{Electrode: Single Walled Carbon Nanotubes (SWCNTs)}

The electrode material plays an important role in determining the capacitance and charge storage capacity of a supercapacitor. Of the many electrode properties which impact capacitance, the specific surface area of the material tends to predominate in determining the capacitance of a supercapacitor. When the material is in contact with an electrolyte, the measured interfacial capacitance of different materials does not linearly increase with the specific surface area because not all available surface area is electrochemically accessible. ${ }^{25}$ The effective pore size of the electrode material plays a vital role in setting the electrochemically accessible area. Research has shown that when the effective 
pore size is very close to the size of the solvated ion, the maximum double-layer capacitance is observed. ${ }^{28}$ Carbon material has the following properties: (1) high specific area, (2) good intra- and inter- particle conductivity for porous matrices, and (3) good electrolyte accessibility to the interpore space of carbon materials, which makes them good electrodes for supercapacitor. ${ }^{1}$

In this research, we have chosen to study single-walled carbon nanotubes (CNTs). CNTs have significantly advanced the science and engineering of carbon materials due to their physical and chemical properties ${ }^{17}$ CNTs are rolled up graphene sheets as seen in Figure $2.1^{29}$ and depending upon the number of layers, they are classified into single-walled carbon nanotubes (SWCNTs) and multi-walled carbon nanotubes (MWCNTs). SWCNTs have proven themselves as a promising electrode of choice in electrochemical energy conversions and storage because of good electrical conductivity, unique pore structure, good thermal stability, relatively low cost, good corrosion resistance, and readily accessible surface area ${ }^{30}$. They are also a good support for active materials due to their high mechanical stability and open tubular network. 


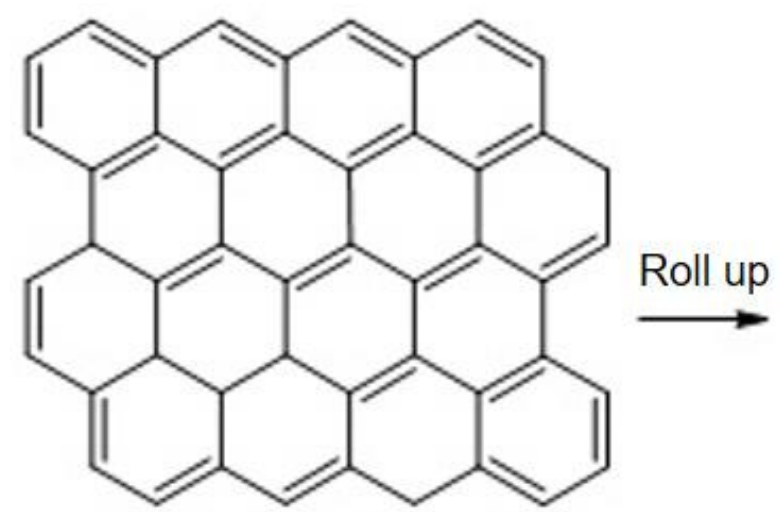

graphene sheet

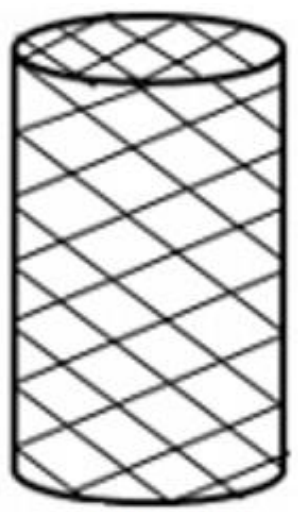

SWCNT

Figure 2.1 Schematic of a portion of a graphene sheet rolled up to a SWCNT.

Nanotube networks use almost all of their available surface to contact the solution. Therefore, SWCNTs have proven to accumulate large amounts of interfacial charge and have accessible mesopores formed by their entanglement and by the central canal. ${ }^{17}$ Furthermore, for fully de-aggregated SWCNT specimens, there is an easy diffusion of electrolyte ions through the mesoporous network. They have very low electronic conductivity, suggesting that SWCNT supercapacitors have a very low effective internal resistance (ESR or equivalent series resistance). ESR reflects the potential required to transport ions within the matrix of the supercapacitor. Thus, if SWCNT supercapacitors are properly solubilized and do not aggregate extensively in the solid state, they may exhibit high energy and power densities. ${ }^{17,31}$

To reduce the ESR and increase the surface area even further, this research work has focused on covalent grafting of the polymer electrolyte polyethylene glycol to the SWCNTs. It is expected that the covalent attachment of the PEG to 
the SWCNTs will further increase the ion mobility from the electrolyte to the current collector.

\subsection{Electrolyte: Polyethylene Glycol (PEG) - Ferrocene dicarboxylic acid - $\mathrm{LiClO}_{4}$}

The electrolyte consists of salt and solvent, an essential component of the supercapacitor which provides ionic conductivity and enables charge compensation between electrodes in the cell. The electrolyte also plays an important role in the formation of the electrical double-layer at the interface between electrode and electrolyte. The formation of the double-layer is the reversible process for charge storage and determines the supercapacitor performance..$^{26}$

In this research, a polymer electrolyte solvent (Figure 2.2) was prepared, based on polymerization of the ferrocene-dicarboxylic acid with polyethylene glycol (PEG)-400. The resulting polymer incorporates the redox center into the main chain of the polymer electrolyte, and thus ensures the solubility of the redox moiety. The electrode (SWCNT) grafted polymer electrolyte should have various advantages over the liquid electrolyte such as less leakage, good ionic conductivity, good interfacial contact with the electrode material, and even a degree of mechanical strength. The pre-requisites for the polymer electrolytes are: (1) high ionic conductivity at ambient and sub-ambient temperature, (2) high chemical, electrochemical and thermal stability, (3) appreciable transference number, (4) compatibility with electrodes, and (5) good cycle life.6,32 


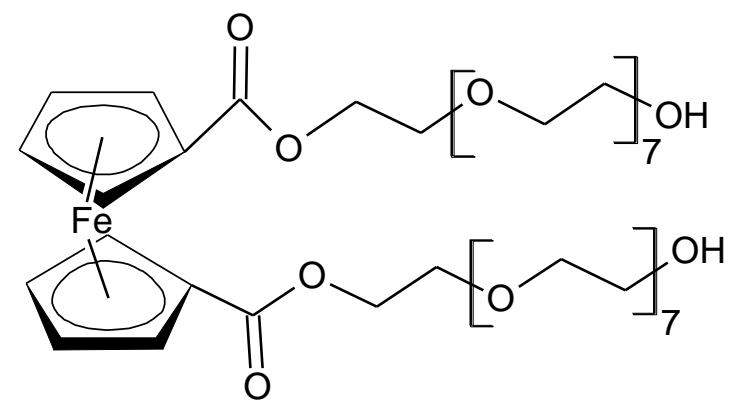

Figure 2.2 Ferrocene dicarboxylic acid - polyethylene glycol polymer

A conventional (non-surface grafted) polymer electrolyte supercapacitor can suffer from a poor electrolyte-electrode contact area. Because of the size of the polymer chains, the ion accessibility to the interstitial electrode surface may be limited as illustrated in Figure 2.3. This Figure illustrates the poor ionic accessibility into the bulk of porous electrode for a polymer electrolyte (Figure 2.3b), which results in the low capacitance.

(a)

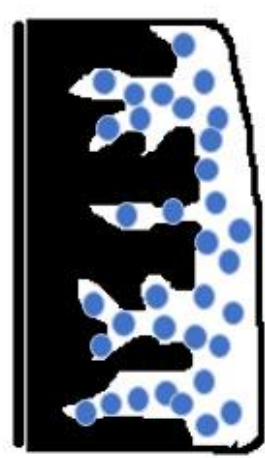

(b)

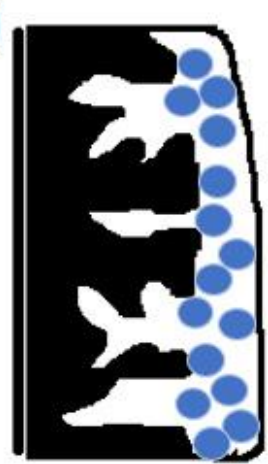

Figure 2.3 Schematic electrolyte ionic accessibility of an electrode in (a) liquid electrolyte and (b) polymer electrolyte.

Finally, to further enhance the charge storage capacity of the supercapacitors, redox active electrochemical species were covalently grafted into the polymer electrolytes. This configuration should form a gel-polymer electrolyte (GPE). ${ }^{26,33}$ GPEs possess cohesive properties of solids as well as diffusive property of liquids. ${ }^{32}$ A GPE is generally composed of a polymer matrix 
(host polymer), and a liquid electrolyte containing conducting salt and a redox species. In order to enhance the mobility of electrolyte ions, a small volume percentage of acetonitrile was added to the polymer phase. The surface grafted GPEs are known to exhibit higher capacitance than the solid-polymer electrolytes. ${ }^{26}$

In this case, the polymer electrolyte solvent used was polyethylene glycol and electrolyte salt was lithium perchlorate. Pure PEG based electrolytes are complexed with the lithium salt. The ionic conductivity is provided by the transport of salt ions through the polymer as shown in Figure $2.4 .^{26}$ It was also found that the lower molecular weight PEG increased the solubility of the crystalline polymer/salt complexes. ${ }^{7}$

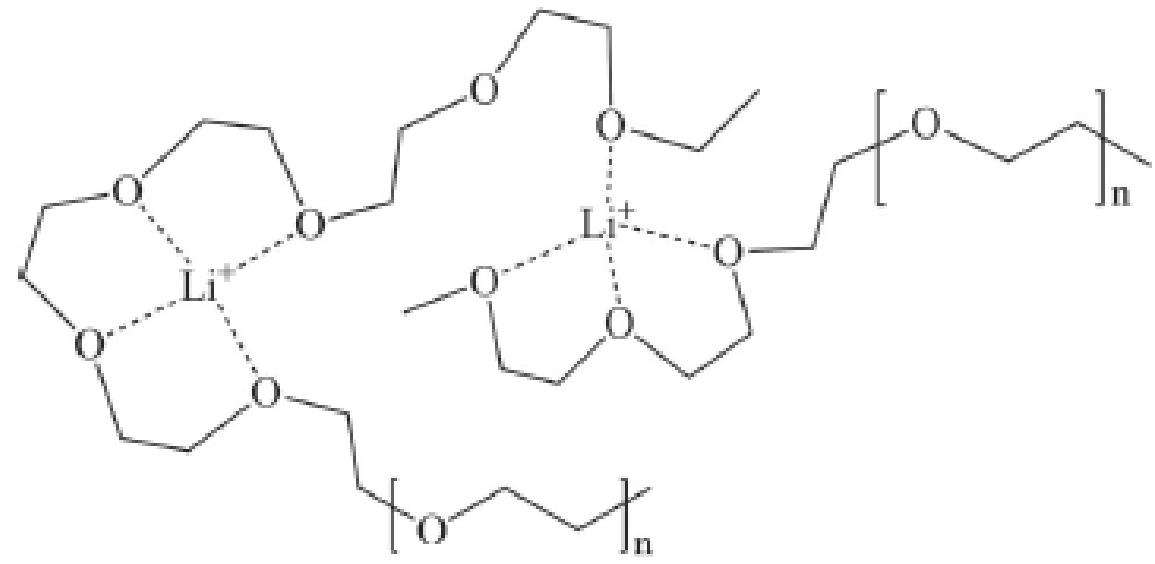

Figure 2.4 Lithium ion complexed in a polymer host.

In this work, redox polymer was made by esterification of the ferrocene dicarboxylic acid (Fc-DCA) with PEG-400. The Fc-DCA has a chemically and kinetically reversible, one electron oxidation at $0.853 \mathrm{~V}$ vs saturated calomel electrode (SCE). Two carboxylic acid groups permit Fc-DCA to copolymerize with 
PEG. Moreover, the redox potential is greater than that of a ferrocene, thus permitting a modest increase in the power density of batteries prepared with it. The redox reaction is shown in Figure 2.5. ${ }^{34}$

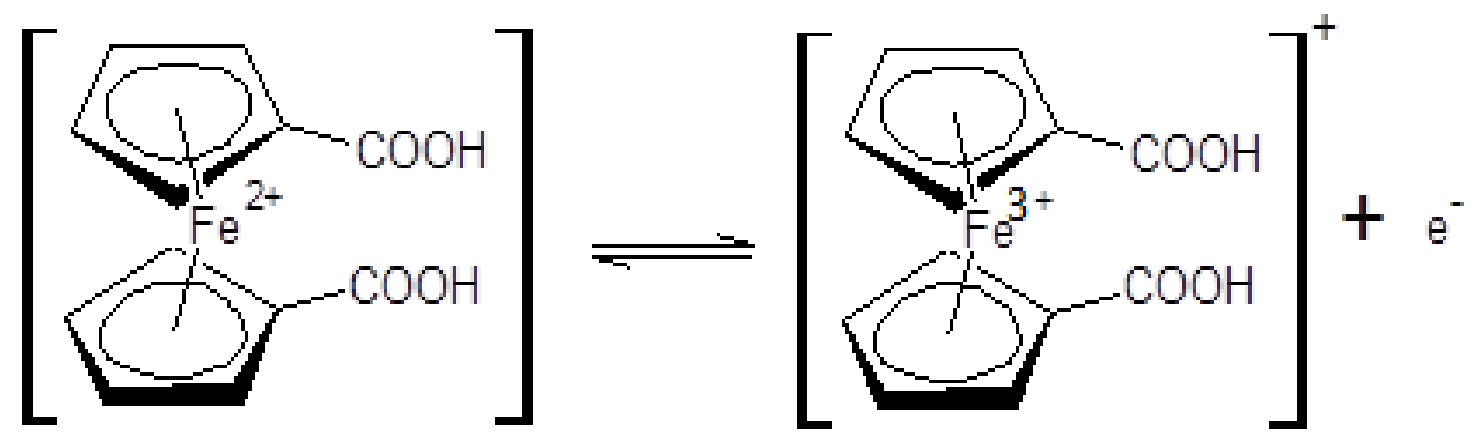

Figure 2.5 Ferrocene dicarboxylic acid redox couple.

To summarize, the covalent grafting of the PEG plays several important roles: (1) to complex with the lithium salts and provide ionic conductivity, (2) to prevent the aggregation of CNTs and thus provide a higher surface area for ion accessibility, and (3) to covalently attached with redox species, therefore reducing their tendency to crystallize, and to become inaccessible electrochemically.

\subsection{Separator}

The separator behaves like an ionic bridge and is located between the two electrodes in a supercapacitor cell. It prevents electrical contact between the two electrodes but is an ion-permeable which allows the ionic charge transfer. The critical requirements for the separators are namely, (1) minimal resistance for an ion transfer within the electrolyte, (2) strong electronic insulating capability, (3) high chemical and electrochemical stability, (4) good mechanical strength to provide device durability, and (5) thin and highly porous films or membranes. A 
porous polycarbonate filter of $0.6 \mu \mathrm{m}$ pore size was used as the separator in this research. 6,35

The synthesis and characterization of the electrode and electrolyte materials for supercapacitors mentioned above are discussed in detail in further chapters. In the last chapter, the fabrication and performance of the hybrid cell is studied and analyzed. 


\section{Chapter 3 : FUNCTIONALIZATION OF CARBON NANOTUBES}

\subsection{Introduction}

The carbon nanotubes are chemically inert like other carbon-based materials such as glassy carbon, graphite, and diamond. Impurities are generated during the production of CNTs, such as byproduct carbonaceous species and residue from the transition metal catalysts, that are used during the synthesis. ${ }^{36}$ Due to transition metal catalysts, CNTs possess magnetic properties ${ }^{37}$ Also, CNTs are typically insoluble in all organic solvents and aqueous solution as they form bundles, due to the strong van der Waals interaction that tightly holds them together. ${ }^{38}$ The overall yield of the usable material is decreased due to the above reasons and this interferes with most of the desired properties. ${ }^{39}$ So, to modify the CNTs, various chemical treatments are performed to give them new chemical and physical properties, for example, increased solubility, higher chemical reactivity, and greater compatibility with polymers. ${ }^{39}$

The CNTs can undergo chemical modifications to enhance their solubility in various solvents. There are two main approaches for the functionalization of CNTs: (1) the covalent attachment of chemical groups through reactions onto the $\pi$-conjugated skeleton of CNTs, and (2) the noncovalent wrapping or adsorption of various functional molecules on the conjugated CNTs. ${ }^{3}$ In covalent functionalization, functional groups are attached to the ends and sidewalls of the tubes. The chemical functionalization/acid treatment shortens the CNTs and incorporates oxygenated groups at open ends and along the sidewall. This type 
of treatment modifies the electronic and structural properties of the CNTs dramatically. ${ }^{40}$ Oxidative chemical treatment covers the ends and sidewalls of the CNTs with oxygen-containing groups such as carboxylate groups, hydroxyl groups, ether groups, etc. For fundamental research on nanotubes and their application in electronic devices, the presence of an oxygenated group on CNTs is important. ${ }^{41}$

In this work, different types of oxidants were used for functionalization of the CNTs. The first acid treatment uses a 3:1 mixture of concentrated $\mathrm{H}_{2} \mathrm{SO}_{4} / \mathrm{HNO}_{3}$ as reported by Liu et al. ${ }^{42}$ to cut the highly tangled long ropes of CNTs into short, open-ended pipes and leave carboxylic groups at the open ends. Oxidizing reagents such as $\mathrm{HNO}_{3}, \mathrm{O}_{3}, \mathrm{KMnO}_{4}, \mathrm{OsO}_{4}$, and $\mathrm{RuO}_{4}$ were also reported for the oxidation of CNTs. ${ }^{41}$ The reaction for the acid treatment is shown in Scheme 3.1. 42

Scheme 3.1 Acid treatment of CNTs
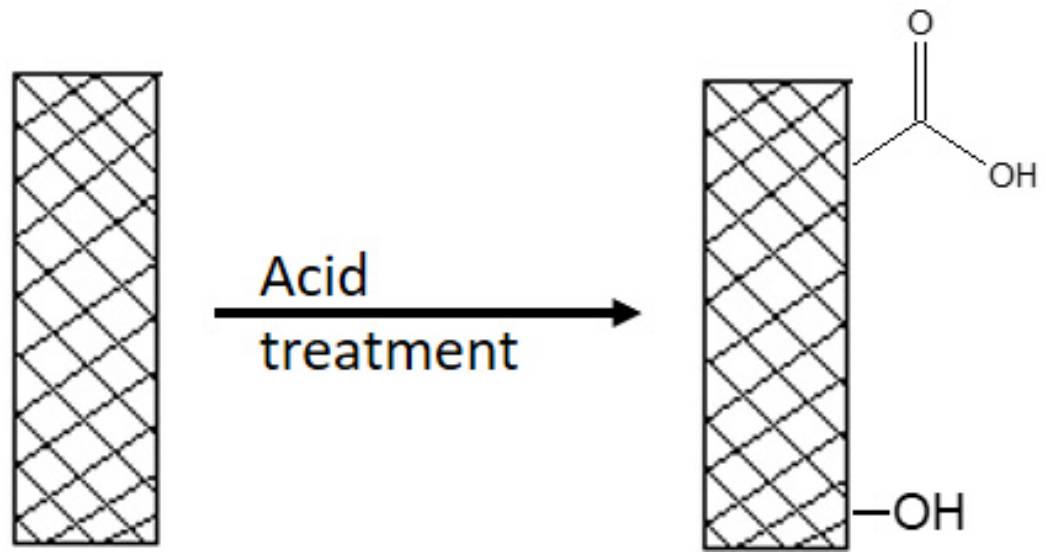

A dry method, utilizing ultraviolet (UV) treatment was also investigated by Cai et al. ${ }^{43}$ for the oxidation of CNTs. UV light produces a more reactive environment 
by exciting ambient oxygen molecules from their triplet spin-triplet state into a spin-singlet state. This facilitates charge transfer from the CNTs to oxygen molecules and promotes their chemical reaction. This UV treatment removes the end caps of the CNTs and introduces a variety of oxygenated functional groups. ${ }^{43}$ Diels-Alder CNT reactions are also reported wherein the CNTs act as dienophile which react with different cyclic dienes, as shown in Scheme 3.2.

Scheme 3.2 The Diels Alder mechanism.
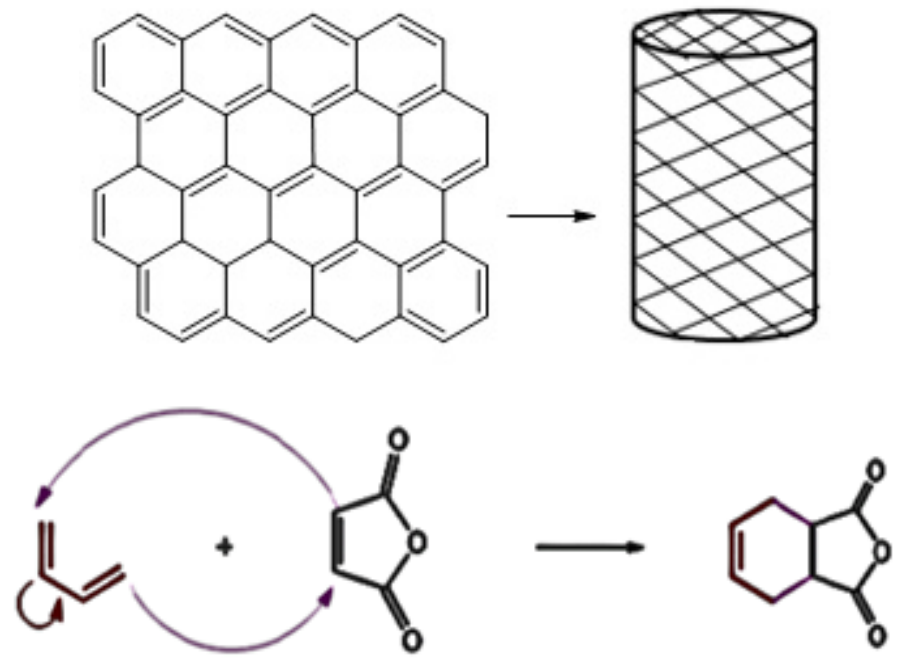

In this chapter, we demonstrate different experiments for the functionalization of CNTs by using, (1) ozone (UV treatment), (2) maleic anhydride (Diels-Alder reaction), (3) a concentrated acid mixture of $\mathrm{H}_{2} \mathrm{SO}_{4}$ and $\mathrm{HNO}_{3}$, and (4) reflux using $13 \mathrm{M} \mathrm{HNO}_{3}$. These reactions are analyzed spectroscopically and are shown to dramatically improve the solubility of CNTs in various solvents. 


\subsection{Materials}

The SWCNTs were purchased from Cheaptubes Inc. These SWCNTs had an average diameter of $1-2 \mathrm{~nm}$ and purity above $90 \%$. The concentrated sulfuric acid (98\%) and concentrated nitric acid (70\%) were obtained from Sigma Aldrich Inc.

\subsection{Experimental Methods}

\subsubsection{UV Ozone Treatment}

The ozone gas was generated in the laboratory using a low-pressure mercury UV lamp and was continuously introduced into a 2-port plastic bottle $(100 \mathrm{~mL})$ which contained $50 \mathrm{mg}$ of SWCNTs. The SWCNTs were exposed to ozone gas for 120 minutes at room temperature and the sample bottle was gently agitated every 10 minutes to provide uniform exposure. The residual ozone gas was scrubbed into a $0.5 \mathrm{M}$ iodide solution. The ozonized SWCNTs were collected and characterized. ${ }^{43}$

\subsubsection{Maleic Anhydride Treatment}

The pristine SWCNTs (30 mg), were added to $20 \mathrm{~mL} \mathrm{~N}$-methyl-2-pyrolidone (NMP) into an oven dried, three-necked flask. Sonication was performed for 1.5 hours to degas the resulting suspension. To this sonicated mixture, $0.15 \mathrm{~g} / \mathrm{mL}$ of maleic anhydride was slowly added. The subsequent mixture was stirred for 48 hours at $170^{\circ} \mathrm{C}$ under a nitrogen atmosphere. The resultant reaction mixture was filtered through a $0.6 \mu \mathrm{m}$ polycarbonate membrane to give a black product. 


\subsubsection{Concentrated Sulfuric/Nitric acid (3:1) Treatment}

The pristine SWCNTs $(20 \mathrm{mg})$, were suspended in $20 \mathrm{~mL}$ of $3: 1$ concentrated acid mixture of $\mathrm{H}_{2} \mathrm{SO}_{4}(98$ wt $\%)$ and $\mathrm{HNO}_{3}(16 \mathrm{M})$ and sonicated for 30 hours in a water bath. The resultant suspension was then diluted with $200 \mathrm{~mL}$ DI water, filtered, and collected on a $0.6 \mu \mathrm{m}$ polycarbonate membrane filter and washed again with DI water. The acid-treated CNTs on the filter membrane were oven dried at $80^{\circ} \mathrm{C}$ for 24 hours. ${ }^{41}$

\subsubsection{M HNO 3 Treatment}

The pristine SWCNTs $\left(200 \mathrm{mg}\right.$ ), were refluxed in a $55 \mathrm{~mL}$ of $13 \mathrm{M} \mathrm{HNO}_{3}$ for four days. $13 \mathrm{M} \mathrm{HNO}_{3}$ was prepared by diluting $16 \mathrm{M} \mathrm{HNO}_{3}$ with DI water. The dried acid treated CNTs were obtained using the same procedure as was used for concentrated sulfuric/nitric acid treatment. ${ }^{41}$

\subsection{Characterization Techniques}

The most common and effective methods to characterize carbon nanotubes are Raman spectroscopy and fourier transform infrared spectroscopy (FTIR).

\subsubsection{Raman Spectroscopy}

Raman spectroscopy is a form of vibrational spectroscopy. It is one of the most powerful techniques for characterization of carbon nanotubes. The Raman effect is based on an inelastic scattering which occurs when a very small fraction ( 1 in $10^{7}$ photons) of incident light is scattered at optical frequencies lower than the incident frequency of photons. When electromagnetic radiation is incident on the molecule, the energy may be transmitted, absorbed, or scattered. A small 
fraction of the scattered radiation encodes vibrational information as described below.

When the incident photon interacts with a molecule in the ground vibrational state, the molecule absorbs energy and is excited into a very short lived so-called virtual state. The molecule immediately decays back to the ground vibrational state and emits a scattered photon whose energy and frequency is same as that of the incident photon, by a process known as the Rayleigh scattering. Whereas, a small proportion of the molecules in the virtual state may decay into an excited vibrational energy level. This type of scattering is referred to as Raman scattering, and arises due to a change in the polarizability of the molecule during the vibration. ${ }^{44}$ So, the Raman scattered photon in this case has less energy than the incident photon. This scattered photon reveals the Stokes-shift which is plotted in the Raman spectrum. The other possibility is the interaction between the incident photon and a molecule already in a vibrationally excited state. When the molecule decays back to the ground vibrational state, energy loss by the emitted photon is greater than the incident photon. This photon emission gives rise to an anti-Stokes shift in the Raman spectrum. The Raman and Rayleigh scattering is illustrated in the Jablonski diagram in Figure 3.1.44 


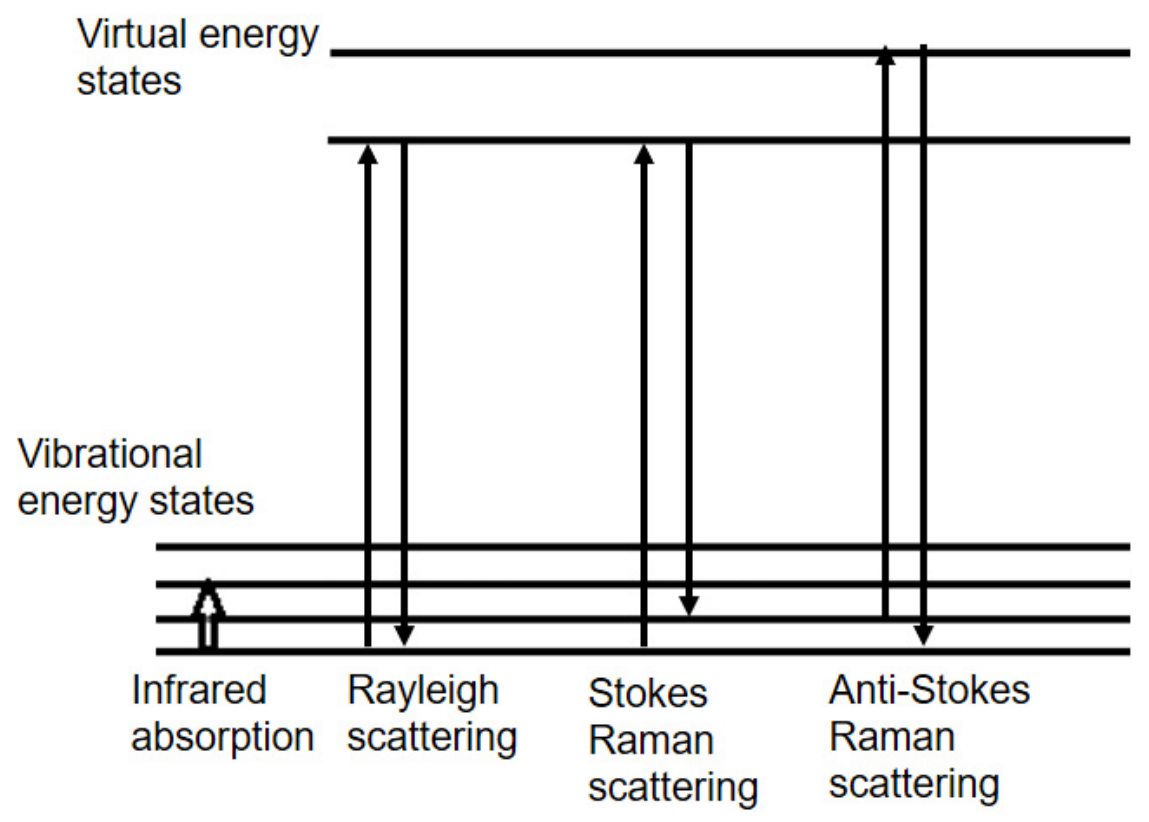

Figure 3.1 Energy Level diagram.

The Raman spectrum is the plot of the intensity of Raman scattered radiation as a function of its frequency difference from the incident radiation (in wavenumbers or $\mathrm{cm}^{-1}$ ), and the difference in wavenumber called as the Raman shift. The peak positions and relative peak intensities in the Raman spectrum provides a unique chemical fingerprint which can be used to identify a molecule. The intensity of the spectrum is directly proportional to the concentration of the scattering molecule.

The presence of $\mathrm{sp}^{3}$ hybridization within the CNTs due to the covalent attachment of functional groups can be detected by the Raman spectroscopy. The characteristic features are the following: (1) a radial breathing mode (RBM) typically found between $100 \mathrm{~cm}^{-1}$ and $400 \mathrm{~cm}^{-1}$ is the vibration characteristic of CNTs and considered as their fingerprint, (2) a high frequency tangential mode (G-band) between 1500 and $1600 \mathrm{~cm}^{-1}$ which is an intrinsic feature of CNTs 
closely related to vibrations in $\mathrm{sp}^{2}$ hybridized graphitic carbon, and (3) a large structure defect mode (D-band) between 1200 and $1350 \mathrm{~cm}^{-1}$ which is characteristic of $\mathrm{sp}^{3}$ hybridization due to the covalent attachment of functional groups. The ratio of intensities of the D-band and the G-band gives information about the covalent functionalization of the sidewalls of CNTs. The higher D/G ratio means the more functional groups are attached to the surface of the CNTs. ${ }^{45,46}$

\subsubsection{Fourier Transform Infrared Spectroscopy (FTIR)}

FTIR is also a form of vibrational spectroscopy. It is an easy way to identify the functional groups in the molecule. Infrared (IR) radiation is passed through the sample, where some of the IR radiation is absorbed by a sample and some of it is transmitted. IR bands arise from change in the dipole moment of the molecule due to the interaction of light with the molecule. The molecular transmission and absorption results in the spectrum creating a molecular fingerprint of the sample.

Attenuated total reflectance (ATR) - FTIR is used in this work. ATR is a widely used FTIR sampling technique which requires little or no sample preparation, thus speeding sample analysis. The main advantage of ATR sampling is that it has a very thin sampling path length and depth of penetration of the IR beam into the sample. The ATR measures the change that occurs in the total internally reflected beam when it is in contact with the sample - this is illustrated in Figure 3.2. The ATR crystal is an optically dense medium, in this 
case $\mathrm{ZnSe}$ crystal, with high refractive index was used, on which an infrared beam is directed at a certain angle. An evanescent wave is formed due to this internal reflectance that extends beyond the surface of the crystal into the sample. The evanescent wave is attenuated in the regions of the infrared spectrum where the sample absorbs energy. This attenuated evanescent wave is passed back to the IR beam which then comes out from the opposite end of the crystal and passed to the detector, where the system generates an infrared spectrum..$^{47,48}$

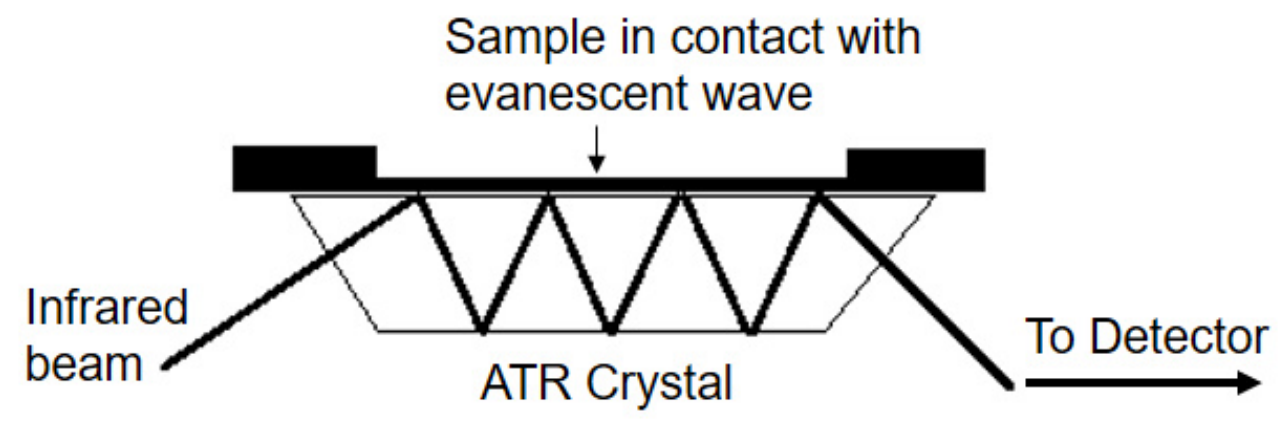

Figure 3.2 A multiple reflection ATR system.

The FTIR spectrum is the plot of an absorbance or percent transmittance versus the frequency between 4000 and $400 \mathrm{~cm}^{-1}$ (wavenumbers). The frequencies of the absorption peaks in the IR can directly be correlated to the bonds within the compound being analyzed. The IR spectroscopy can identify organic functional groups attached on the CNT's surface by measuring characteristic vibrational modes. ${ }^{49}$ The characteristic stretching frequencies of the CNTs on the IR spectra are $\sim 2100 \mathrm{~cm}^{-1}$ related to $\mathrm{C}=\mathrm{C}$ of CNTs, $\sim 3300 \mathrm{~cm}^{-1}$ for hydroxyl group and $\sim 1650 \mathrm{~cm}^{-1}$ for $\mathrm{C}=\mathrm{O}$ functional group. 


\subsection{Results and Discussion}

\subsubsection{Ozonolysis}

The unfunctionalized SWCNTs are insoluble in most of the solvents due to their strong hydrophobic nature. When SWCNTs are functionalized, the oxygenated groups induce polarity into a molecule and stabilize them in the polar solvents by hydrogen bonds and polar-polar interactions. Ozone treated SWCNTs were expected to be soluble in water due to the increased polarity of the molecule. However, the expected result was not seen when they were dispersed in water indicating no incorporation of oxidized defects. The possible explanations for this may be low reaction time or non-uniform exposure of ozone to SWCNTs. To ensure that this was the case, the experiments were repeated where SWCNTs were exposed to ozone for prolonged time and were suspended in DI water. The same results were obtained, even after four hours of ozone exposure with constant stirring.

\subsubsection{Maleic Anhydride}

During the filtration, most of the functionalized SWCNTs product, passed through the polycarbonate membrane filter along with the reaction mixture. The obtained filtrate was black/brown in color which indicated the shortening of SWCNTs due to functionalization. The black colored filtrate suggested that the functionalized SWCNTs had improved solubility. To separate this SWCNT product from the black filtrate solution, it was again filtered using a $0.45 \mu \mathrm{m}$ nylon 
membrane filter. Again, the filtration was not successful, and no product was retained.

After several failed filtration attempts, centrifugation was employed as an alternative method to extract SWCNTs from the mixture. The reaction mixture was centrifuged using a Labnet Mini Centrifuge C-1200 (115 V/60 Hz) at a speed of $2000 \mathrm{rpm}$ for 60 minutes. This procedure was repeated multiple times, still the SWCNTs did not extract from the supernatant solution. This may be due to the low rpm value of the centrifuge instrument, but the reason that tubes were not collected is still not clear.

\subsubsection{Concentrated Sulphuric/Nitric acid (3:1)}

The acid treated tubes were expected to be soluble in water, but the expected result was not seen. The insolubility of treated SWCNTs in the water suggested that no functional groups were attached to the tubes. This may be due to the lack of heating which might have reduced the reaction rate. Therefore, a different approach, a reflux method described below, was employed to oxidize the SWCNTs.

\subsubsection{M $\mathrm{HNO}_{3}$}

A solubility test was carried out to confirm the functionalization of the SWCNTs treated with $13 \mathrm{M} \mathrm{HNO}_{3}$. The acid-refluxed SWCNTs were soluble in water after sonication for 5 mins as shown in Figure 3.3. This indicated that the reflux nitric acid had incorporated oxygenated groups in the tubes which have 
increased the hydrophilicity and reactivity of the SWCNTs. Raman and FTIR spectroscopies were used to further characterize the SWCNTs.

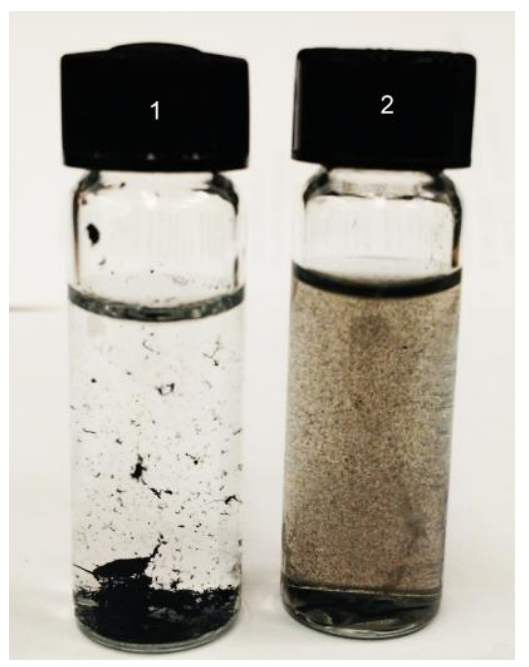

Figure 3.3 Solubility test in water (1) pristine and (2) refluxed SWCNTs.

The Raman spectra of the pristine SWCNTs and the refluxed SWCNTs were recorded on the Enwave Optronics Raman Spectrometer using a $785 \mathrm{~nm}$ laser. The Raman spectra is shown in Figure 3.4. The spectra for the pristine SWCNTs showed prominent G-band at $\sim 1615 \mathrm{~cm}^{-1}$ wavelength attributed to the intrinsic feature of the tubes closely related to the $\mathrm{sp}^{2}$ vibrations. In addition, the SWCNTs treated with nitric acid showed a distinct D-band at $\sim 1320 \mathrm{~cm}^{-1}$ along with Gband. This D-band was due to defects on the SWCNTs caused by the acid treatment, which breaks the symmetry of SWCNTs. The D/G ratios of pristine and refluxed SWCNTs were calculated to be 0.08 and 0.15 respectively. The higher D/G ratio of treated SWCNTs suggested successful functionalization as the ratio is sensitive to the presence of $\mathrm{sp}^{3}$ carbons on the SWCNTS surface. 


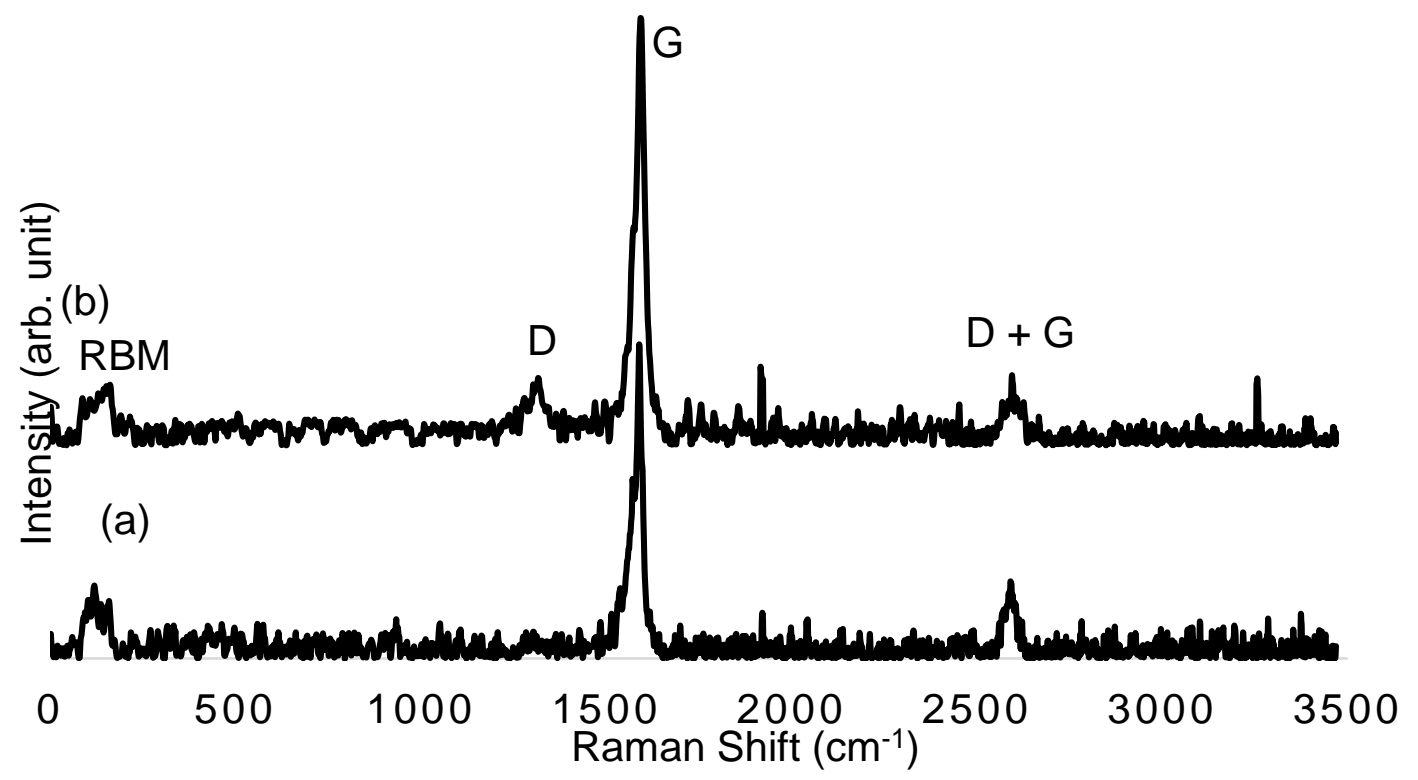

Figure 3.4 Raman spectra of (a) pristine and (b) refluxed SWCNTs.

The FTIR spectra of pristine and refluxed SWCNTs were taken on the Thermo Nicolet 6700 FT-IR spectrometer as shown in Figure 3.5. Similar absorption bands at $\sim 2120 \mathrm{~cm}^{-1}$ were observed for both the refluxed and the pristine SWCNTs indicating the $\mathrm{C}=\mathrm{C}$ vibrational frequency in the SWCNTs structure. The additional bands were observed in the acid treated SWCNTs spectra at $\sim 3350 \mathrm{~cm}^{-1}$ and $\sim 1640 \mathrm{~cm}^{-1}$, were related to $\mathrm{O}-\mathrm{H}$ (hydroxyl group) and $\mathrm{C}=\mathrm{O}$ (carboxyl group) respectively, which were consistent with the known vibrational values of the functional groups. Thus, the presence of the $\mathrm{O}-\mathrm{H}$ and the $\mathrm{C}=\mathrm{O}$ stretch modes corresponding to hydroxyl and carboxylic acid groups in the acid treated SWCNTs spectra suggested successful functionalization of the nanotubes by $13 \mathrm{M} \mathrm{HNO}_{3}$. 


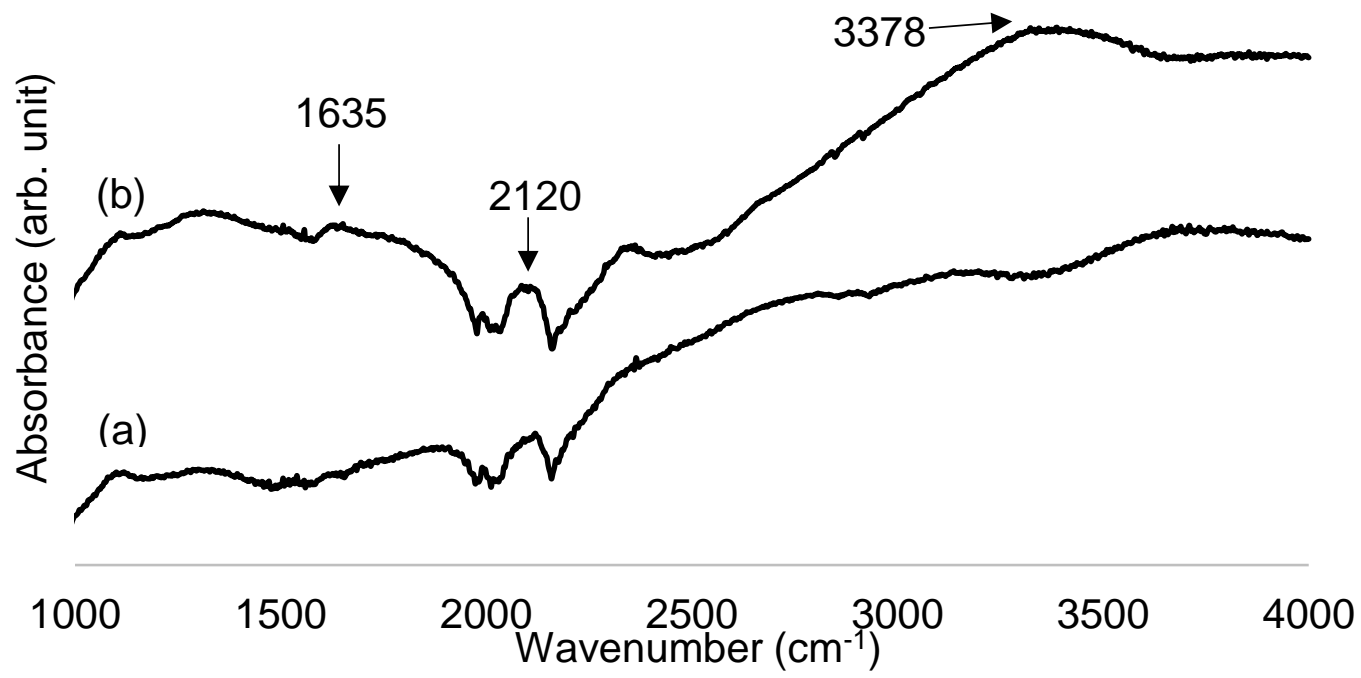

Figure 3.5 FTIR spectra of (a) pristine and (b) refluxed SWCNTs.

\subsection{Conclusions}

Based on the above results, it was found that the reflux with $\mathrm{HNO}_{3}$ was the most effective method for functionalization of the CNTs. This suggested that the heating and longer time are important factors in functionalizing nanotubes. The acid refluxed SWCNTs were soluble in water as compared to the pristine ones. The Raman spectra of treated SWCNTs showed distinctive D-band at $1300 \mathrm{~cm}^{-1}$ which is due to the defects in nanotubes. Additionally, the absorption peaks for carboxyl $\left(1640 \mathrm{~cm}^{-1}\right)$ and hydroxyl $\left(3350 \mathrm{~cm}^{-1}\right)$ groups in the FTIR spectra also confirmed the attachment of the functional groups. The solubility test, Raman and FTIR spectroscopy supported the covalent attachment of carboxylic acid and hydroxyl groups on SWCNTs. 


\section{Chapter 4 : SYNTHESIS OF POLYETHYLENE GLYCOL-FERROCENE DICARBOXYLATE (FC-PEG) POLYMER}

\subsection{Introduction}

Ferrocene is a well-known organometallic compound with a unique sandwichlike structure as shown in Figure 4.1. Ferrocene can be oxidized to ferricenium cation $\left(\mathrm{Fe}^{+3}\right)$ with excellent electrochemical kinetics and chemical reversibility. Recently, several attempts to use ferrocene-based organic molecules for active cathode materials have been reported. Ferrocene groups have been covalently incorporated in the polymer for improving the charge transfer between the electrode-electrolyte interface. The ferrocene-substituted poly(pyrrole) and poly(aniline) are also being investigated in batteries, which has resulted in high coulombic efficiencies and low capacity decay within 100 cycles due to the reversibility of the ferrocene. ${ }^{50,51}$

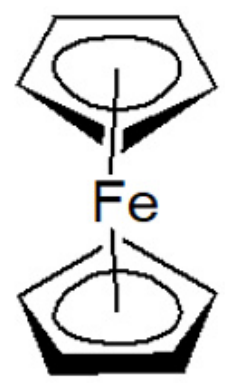

Figure 4.1 Structure of Ferrocene.

In this research, ferrocene-dicarboxylic acid is covalently incorporated in the polyethylene glycol (PEG) host polymer, forming a polyester polymer. As discussed in Chapter 2, when redox species are introduced in the polymer phase, the performance of the supercapacitor may be enhanced. In fact, the covalent attachment of the redox species onto the polymer reduces their 
tendency to crystallize and thereby increases their solubility in the polymer solution. The different types of esterification reactions that were attempted for the synthesis of the redox additive polymer are acid chloride esterification and Steglish esterification method.

The first method is the acid chloride esterification. The nucleophilic addition/ elimination reaction between acid chloride and alcohol yields an ester. First, the carboxylic acid is converted to the respective acid chloride. Different types of reagents can be used for this reaction, including phosphorous $(\mathrm{V})$ chloride, phosphorous (III) chloride, thionyl chloride, and oxalyl chloride. In this work, oxalyl chloride is used as a reagent for the synthesis of the acid chloride in presence of dimethylformamide (DMF). The mechanism for acid chloride formation is through the Vilsmeier-Haack reagent illustrated in Figure 4.2. Once the acid chloride is formed, the second step is the nucleophilic addition/elimination reaction between acid chloride and alcohol which involves three stages. The first stage, is the nucleophilic attack by oxygen of an alcohol molecule on the electropositive carbonyl of an acid chloride, forming a carbonoxygen bond. The second stage is the elimination of a chloride ion and reformation of a carbon-oxygen double bond. The last stage is the deprotonation which forms hydrochloric acid and the desired ester. 

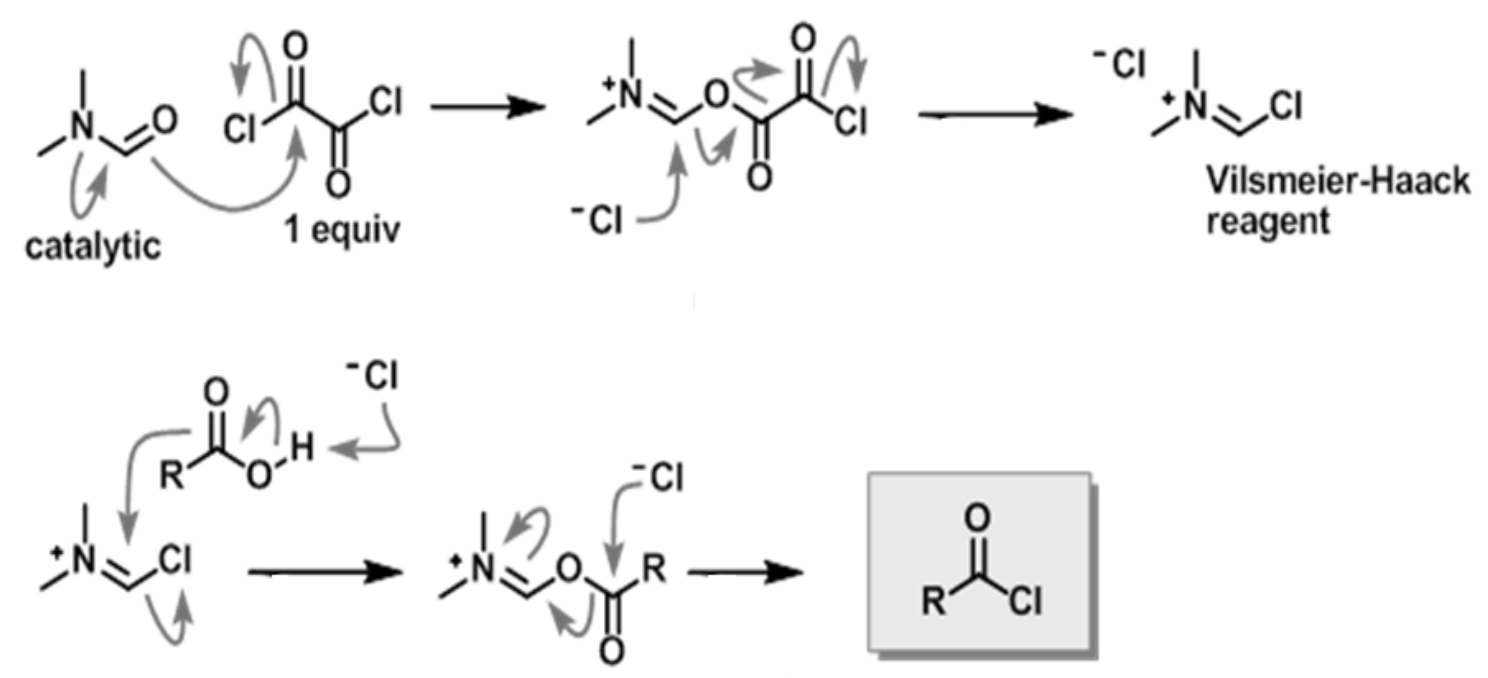

Figure 4.2 Mechanism of the acid chloride formation.

The second method is the Steglish esterification which is a mild reaction and allows conversion of sterically demanding and labile compounds. First, an Oacylisourea intermediate is formed from DCC (dicyclohexylcarbodiimide) and carboxylic acid. The intermediate offers reactivity similar to the corresponding carboxylic acid anhydride. Then the alcohol reacts with an activated carboxylic acid to form the stable dicyclohexylurea (DCU) and the ester. The mechanism is shown in Figure 4.3.52,53 


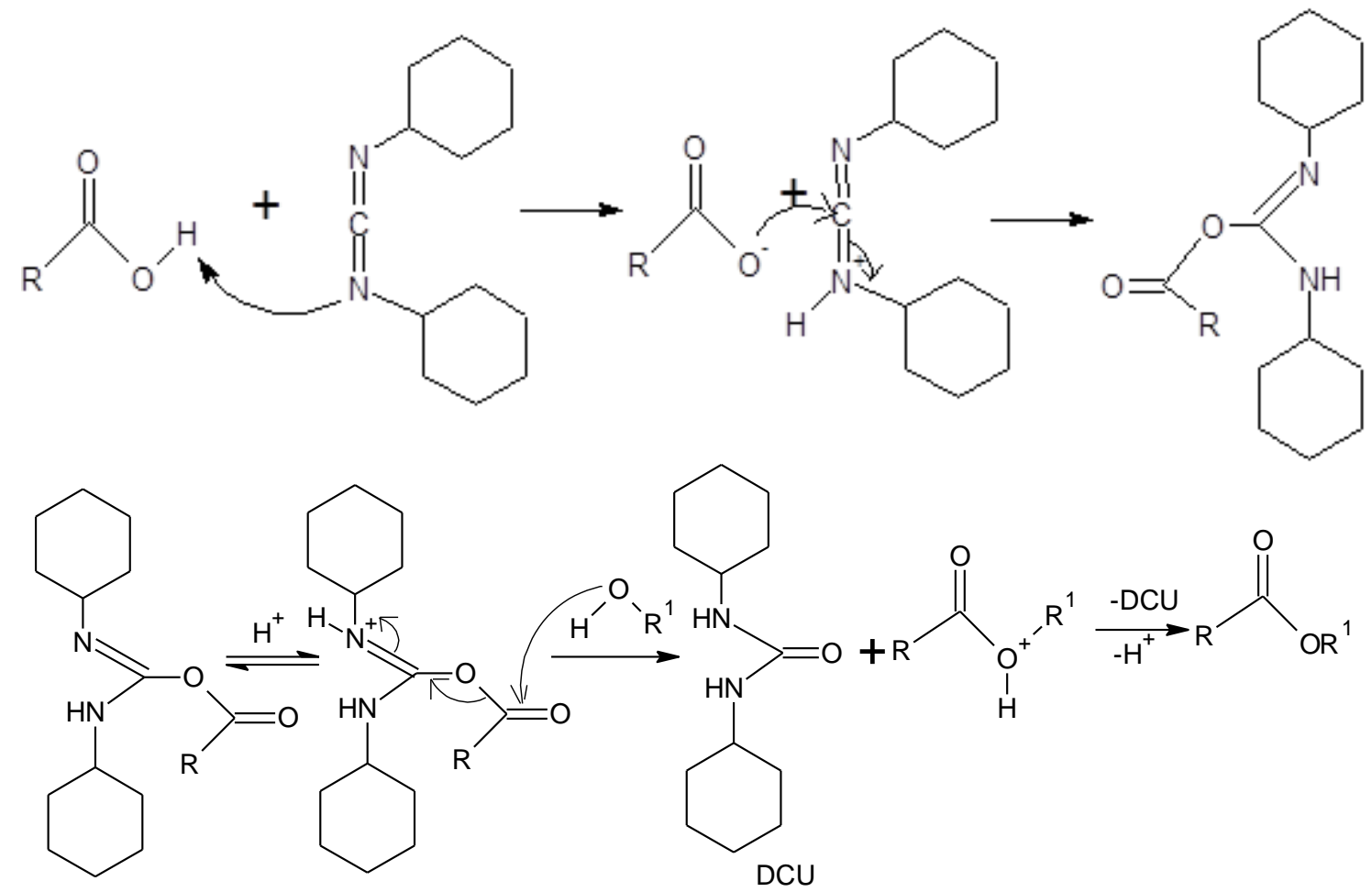

Figure 4.3 Mechanism of the Steglish Esterification.

In this chapter, several experiments are described. Scheme 4.1 shows the acid chloride esterification of PEG and 1,1'-ferrocenedicarboxylic acid with oxalyl chloride as chlorinating agent and DMF or dichloromethane (DCM) as solvent. Scheme 4.2 shows the Steglish esterification of PEG and 1,1'-ferrocene dicarboxylic acid in the presence of DCC or DIC (diisopropylcarbodiimide). The catalyst and solvent used in the reaction were TEA (triethylamine) and DCM, respectively. In these experiments, the degree of polymerization was not monitored. Therefore, different types of FC-PEG polymer were produced, as shown in Figure 4.4. 
Scheme 4.1 Reaction scheme of the acid chloride esterification.

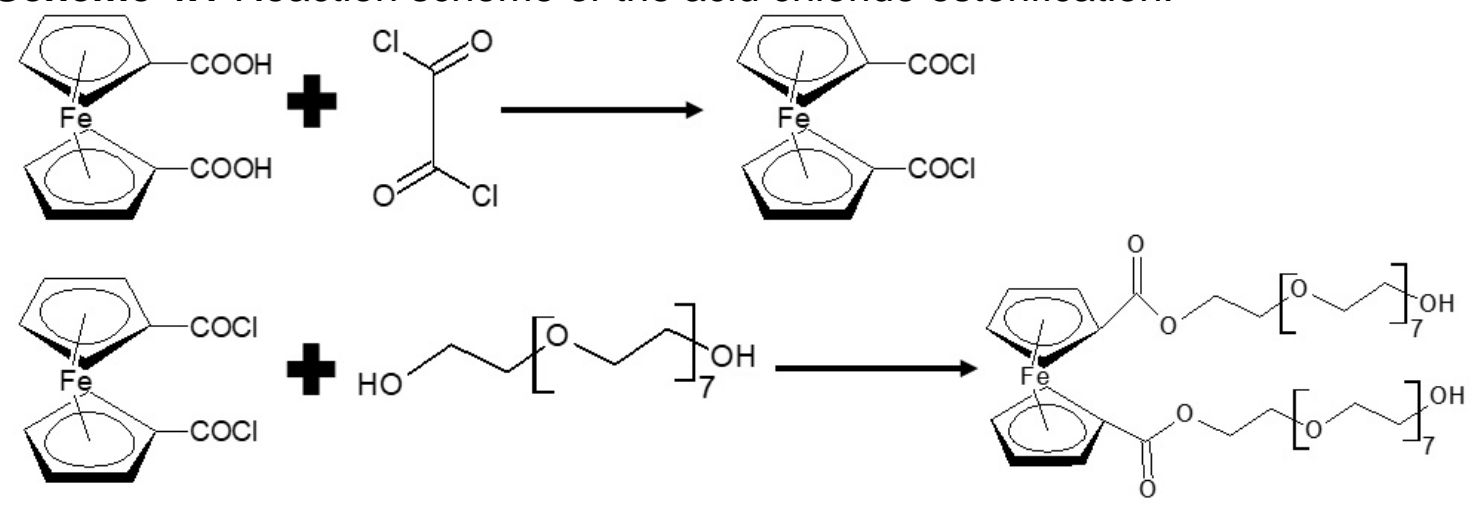

Scheme 4.2 Reaction scheme of the Steglish esterification.
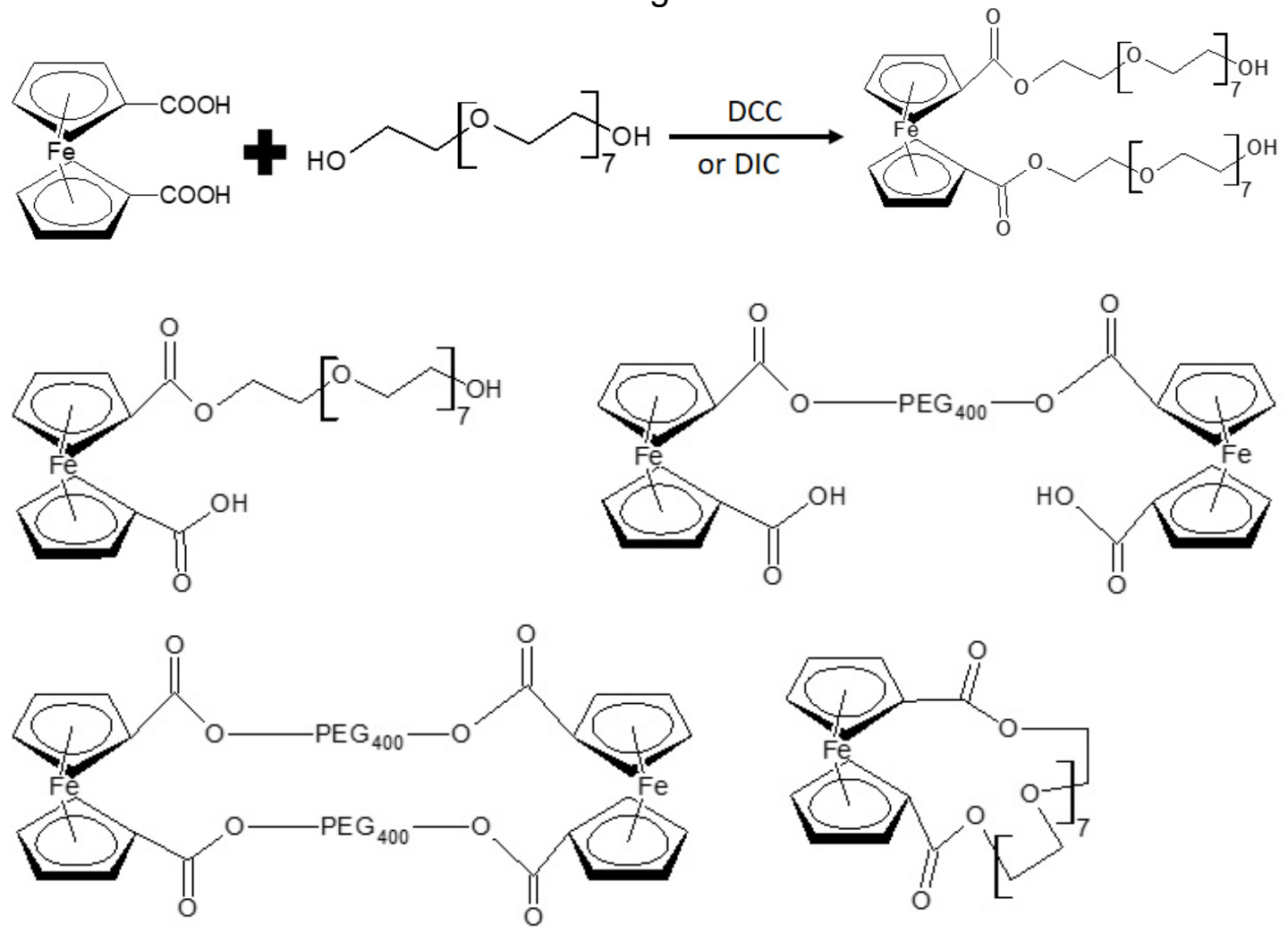

Figure 4.4 Different types of Fc-PEG polymer.

\subsection{Materials}

All the reactions were performed in a three-neck round bottom flask fitted with a rubber septum under a nitrogen atmosphere. All liquid reagents and solvents 
were dried under vacuum and stored over molecular sieves in the refrigerator. The glass syringes were used to transfer dried liquid reagents and solvents into the reaction mixture.

\subsection{Experimental Methods}

\subsubsection{Acid Chloride Esterification}

Ferrocene-dicarboxylic acid $(0.18 \mathrm{mmol}, 50 \mathrm{mg})$, was dissolved in $20 \mathrm{~mL}$ of DCM in a three-necked flask. Then, oxalyl chloride $(30 \mu \mathrm{L})$ was added slowly in the above solution under a nitrogen atmosphere at $0^{\circ} \mathrm{C}$. The reactant mixture was stirred at $0^{\circ} \mathrm{C}$ for 2 hours and then stirred for 8 hours at room temperature. PEG-400 (0.5 mL) was added to the above reactant solution and stirred at room temperature for 48 hours. The resultant suspension was filtered, and filtrate was evaporated to dryness. The precipitation of the ester was done using $10 \% \mathrm{NaOH}$ $(10 \mathrm{~mL})$ and $\mathrm{DCM}(10 \mathrm{~mL})$. The $\mathrm{NaOH}$ layer dissolved the unreacted ferrocenedicarboxylic acid and the DCM layer was separated out. The DCM layer was then evaporated to dryness to get the desired ester product. ${ }^{54}$ However, this method did not give a product, possibly because the solvent was not dried. As a result, when oxalyl chloride was reacted with the ferrocene-dicarboxylic acid, instead of activating acid groups, oxalic acid was formed. Moreover, the PEG was also not dried and water in it might have hydrolyzed ferrocene-diacid chlorides. As the oxalyl chloride is very reactive and sensitive to air, a different one pot approach was employed for the esterification. 


\subsubsection{Steglish Esterification using Dicyclohexylcarbodiimide (DCC)}

PEG-400 (1.1 mmol, $0.44 \mathrm{gm})$, ferrocene-dicarboxylic acid (1 mmol, $0.28 \mathrm{gm}$ ) and TEA (2 mmol, $0.20 \mathrm{gm}$ ) were dissolved in $15 \mathrm{~mL}$ of anhydrous DCM under a nitrogen atmosphere. A solution of DCC (2 mmol, $0.42 \mathrm{gm})$ and $5 \mathrm{~mL}$ of anhydrous DCM was then added dropwise to the flask at $0^{\circ} \mathrm{C}$. The reaction was then stirred for 48 hours at $25^{\circ} \mathrm{C}$ under a nitrogen atmosphere. The mixture was filtered, and the filtrate was evaporated to dryness. The solid was redissolved in $20 \mathrm{~mL}$ of chloroform. The solution was extracted with the $\mathrm{NaOH}$ solution $(0.00375 \mathrm{M})$ and washed with DI water to remove unreacted ferrocenedicarboxylic acid and water-soluble byproducts. The solution was dried over anhydrous sodium sulfate and then evaporated to dryness to yield the brown product. ${ }^{53,55,56}$ But the NMR spectra did not show ferrocene-dicarboxylate peaks. This may be due to the $\mathrm{NaOH}$ washing step, which might have hydrolyzed the polymer, dissolving ferrocene-dicarboxylic acid in the aqueous phase. Therefore, in the next synthetic attempt, $\mathrm{NaOH}$ washing was not given and DIC was used instead of DCC as it is hard to remove a DCU byproduct from the reaction mixture.

\subsubsection{Steglish Esterification using Diisopropylcardodiimide (DIC)}

PEG-400 (1 mmol, $0.4 \mathrm{gm}$ ), ferrocene-dicarboxylic acid (1 mmol, $0.28 \mathrm{gm}$ ), and TEA ( $1 \mathrm{mmol}, 0.1 \mathrm{gm}$ ) were dissolved in $10 \mathrm{~mL}$ of anhydrous DCM under a nitrogen atmosphere. DIC ( $1 \mathrm{mmol}, 0.12 \mathrm{gm})$ was dissolved in $5 \mathrm{~mL}$ of anhydrous DCM and the same reaction procedure was followed as mentioned above. The 
solution was extracted twice with DI water to remove unreacted ferrocenedicarboxylic acid and water-soluble byproducts. The solution was then evaporated to dryness to give the desired yellow product. ${ }^{53,55,56}$ This ester product was then used for characterization and further experiments.

\subsection{Characterization Techniques}

Common and effective methods to characterize organic compounds are NMR (nuclear magnetic resonance) and FTIR. NMR spectra was recorded on a Varian $400 \mathrm{MHz}$ spectrometer and FTIR was recorded on a Thermo Nicolet $6700 \mathrm{FT}-\mathrm{IR}$ spectrometer. To judge the electrochemical behavior of the redox-PEG copolymer or Fc-PEG polymer, an electrochemical technique - cyclic voltammetry was employed using the $\mathrm{CH} 660$ Electrochemical Analyzer. The techniques are briefly described in later sections.

\subsubsection{Nuclear Magnetic Resonance (NMR)}

NMR is the preeminent technique for determining the structure of organic compounds. It identifies the carbon-hydrogen framework of an organic compound. NMR deals with the nucleus of an atom that possess a magnetic moment. When external magnetic field is applied, nuclei align themselves into two spin states: $+1 / 2$ spin state is aligned with the magnetic field which is in a lower energy state and $-1 / 2$ spin state is opposed to the magnetic field which is in a higher energy state. The energy difference between the two states is $\Delta \mathrm{E}$ which is dependent on the applied magnetic field. When radiation with an energy $\Delta \mathrm{E}$ is incident on the sample, the nuclei undergo relaxation. During this process, they 
emit electromagnetic signals which are read by an NMR spectrometer and plotted on the graph of signal frequency versus intensity. ${ }^{57}$

\subsubsection{1 ${ }^{1} \mathrm{H}-\mathrm{NMR}$}

Each group of chemically equivalent proton gives rise to a unique signal. Chemically equivalent protons are protons in the same environment - i.e. protons on carbons with identical bonds. Hence, the number of signals in the NMR spectrum determines the number of non-equivalent protons in a molecule. The chemical shift is the position of a signal in a NMR spectrum (with respect to a reference compound) and is measured in ppm (parts per million). The reference compound used usually is tetramethylsilane (TMS), and TMS protons are given a zero position at the far right of the spectrum, and ppm becomes larger as it moves towards left. The formula for the chemical shift is:

$$
\text { Chemical Shift }(\delta)=\frac{\vartheta_{\text {sample }}-\vartheta_{\text {reference }}}{\vartheta_{\text {reference }}} \quad \text { Equation } 4.1
$$

where $\vartheta_{\text {reference }}$ is frequency of TMS and $\vartheta_{\text {sample }}$ is frequency of sample.

NMR gives information on the relative number of protons and the types of protons in a molecule. The peak integration is proportional to the number of protons that give rise to the signal. The splitting of the proton signals tells the number of protons bonded to adjacent carbons. And the overall chemical shift of a proton gives information about the type of carbon (or another atom) that it is bonded to. Proton splitting follows the $\mathrm{N}+1$ rule where $\mathrm{N}$ is the number of 
equivalent protons that are bonded to adjacent carbons. The number of signals in the spectrum is one more than the number of equivalent protons..$^{57,58}$

\subsubsection{2 ${ }^{13} \mathrm{C}-\mathrm{NMR}$}

${ }^{13} \mathrm{C}-\mathrm{NMR}$ is used, when significant portions of a molecule lack $\mathrm{C}-\mathrm{H}$ bonds and no information can be interpreted with the ${ }^{1} \mathrm{H}-\mathrm{NMR}$. In the ${ }^{13} \mathrm{C}-\mathrm{NMR}$, also each structurally distinct carbon displays a single sharp peak. Unlike ${ }^{1} \mathrm{H}-\mathrm{NMR}$, there is no splitting of peaks in ${ }^{13} \mathrm{C}-\mathrm{NMR}$. This is because the relative abundance of ${ }^{13} \mathrm{C}$ is only $1.1 \%$, so the probability of having two ${ }^{13} \mathrm{C}$ atoms right next to each other is only $0.012 \% .{ }^{59}$

To confirm the chemical structure of Fc-PEG polymer, ${ }^{1} \mathrm{H}-\mathrm{NMR}$, and ${ }^{13} \mathrm{C}-\mathrm{NMR}$ were used. The chemical shifts $(\delta)$ were reported in ppm relative to the residual solvent deuterated dimethyl sulfoxide (DMSO).

\subsubsection{Electrochemical Analysis}

Cyclic voltammetry (CV) is an electrochemical technique for investigating the electrochemical behavior of analytes which can be electrochemically oxidized or reduced. In this technique, the potential applied to the working electrode is varied and the current is measured as a function of potential. The cyclic voltammogram is a plot of current versus potential and indicates the potential at which redox process occurs. To carry out a reduction process, the potential is scanned negatively, and electroactive species gain an electron giving rise to a cathodic peak current $\left(\mathrm{ipc}_{\mathrm{pc}}\right)$ and corresponding peak potential is cathodic peak potential $\left(E_{p c}\right)$. When all the substrate at the surface of the electrode has been reduced, 
the current falls and the potential is reversed and scanned positively leading to an oxidation process. This gives an oxidation peak at anodic peak current ( $\left.\mathrm{i}_{\mathrm{pa}}\right)$ and anodic peak potential $\left(\mathrm{E}_{\mathrm{pa}}\right){ }^{60}$

A three-electrode cell was used to perform the cyclic voltammetric experiments and potentiostat for making the measurements. Three-electrode configuration measures only the properties of the working electrode, and the resistance of reference and auxiliary are cancelled. The principle of the potentiostat is to maintain the constant potential difference between the working electrode and the sum of a programmed potential and the reference electrode potential. ${ }^{61}$

The working electrode is the electrode under study, where the potential is controlled, and the current is measured. The potential is measured against a reference electrode, which maintains a constant potential. The working electrode should neither oxidize nor reduce solvent or supporting electrolyte in a wide potential range. The size and shape of the electrode surface affects the voltammetric response of the electrode. There are two types of electrodes, microelectrodes and ultramicroelectrodes. Microelectrodes are electrodes with a diameter greater than $100 \mu \mathrm{m}$ and less than about $1 \mathrm{~cm}$ and produce currents in microamp to milliamp range. The mass transport within the diffusion layer at a microelectrode occurs mostly perpendicular to the surface (planar diffusion). The peak current follows Randles-Sevcik equation which is as follows: 


$$
\mathrm{i}_{\mathrm{p}}=0.4463 \mathrm{nFAC}\left(\frac{\mathrm{nFvD}}{\mathrm{RT}}\right)^{\frac{1}{2}} \quad \text { Equation } 4.2
$$

where $i_{p}$ is the maximum current in amperes, $n$ is the number of electrons transferred in the redox event, $\mathrm{F}$ is the Faraday constant, $\mathrm{C}$ is the bulk concentration of electroactive substance, $A$ is the electrode area in $\mathrm{cm}^{2}, D$ is the diffusion coefficient in $\mathrm{cm}^{2} / \mathrm{s}$, and $\mathrm{v}$ is the scan rate in V/s. According to this equation, the peak current $\left(i_{p}\right)$ is proportional to the square root of scan rate $(v)$ and concentration of electroactive species. ${ }^{62,63}$ Whereas, electrodes with dimension less than $100 \mu \mathrm{m}$ are known as ultramicroelectrodes, which produce currents typically in the picoamp to nanoamp range. The mass transport at an ultramicroelectrode occurs via radial diffusion. This results into a sigmoidal, steady-state voltammogram. The limiting plateau current from $\mathrm{CV}$ is given by

$$
\mathrm{i}_{\mathrm{lim}}=4 \mathrm{nFrDC} \quad \text { Equation } 4.3
$$

where $\mathrm{i}_{\lim }$ is the limiting current in amperes, $\mathrm{n}$ is the number of electrons transferred in the redox event, $\mathrm{F}$ is the Faraday constant, $\mathrm{r}$ is the electrode radius in $\mathrm{cm}, \mathrm{D}$ is the diffusion coefficient in $\mathrm{cm}^{2} / \mathrm{s}$, and $\mathrm{C}$ is the bulk concentration of electroactive substance. It is possible to measure current reliably in a resistive media with ultramicroelectrodes because the current is very small, and the hemispherical nature of the charge transport makes the effective voltage drop much less significant. ${ }^{62,63,64}$ Examples of different types of working electrodes include mercury, carbon, platinum and gold. 
The reference electrode serves as a stable potential against which the potential of a working electrode may be varied. The electronics of a potentiostat holds the cell potential at programmed value measurements. Examples of reference electrodes include silver/silver chloride, saturated calomel, mercury/mercurous oxide, and mercury/mercury sulfate electrodes. In addition, a simple, bare silver wire is often used as a quasi-reference in non-aqueous environment. The silver wire potential is reasonably stable and can be internally standardized using the ferrocene/ferricenium. ${ }^{64}$

The auxiliary electrode completes the current path in the three-electrode system. This electrode is introduced in the electrochemical cell to prevent the flow of current through the reference electrode. The most commonly used auxiliary electrode is the platinum wire. ${ }^{64}$

A CH 660 Electrochemical analyzer was employed for the CV experiments. In all the experiments performed, $0.1 \mathrm{M}$ lithium perchlorate in anhydrous acetonitrile was used as an electrolyte. The working electrodes were polished first with 1 , 0.3, and 0.05-micron size alpha alumina paste to expose the electrode tip. The CV experiments were performed in solution and semi-solid phases.

\subsubsection{Solution Phase Electrochemistry}

In this method, the ferrocene-dicarboxylic $(3 \mathrm{mM})$ was dissolved in $0.1 \mathrm{M}$ $\mathrm{LiClO}_{4}$ in anhydrous acetonitrile $\left(\mathrm{CH}_{3} \mathrm{CN}\right)$ electrolyte and was analyzed using a conventional microelectrode as well as an ultramicroelectrode. The platinum micro $(3 \mathrm{~mm})$ and ultramicro $(10 \mu \mathrm{m})$ working electrodes, the $\mathrm{Ag} / \mathrm{AgCl}$ reference 
electrode and the platinum wire as an auxiliary electrode were used for the measurements. The electrolyte solution being investigated was purged with helium gas to make the environment oxygen free. The experimental setup is shown in Figure 4.5.

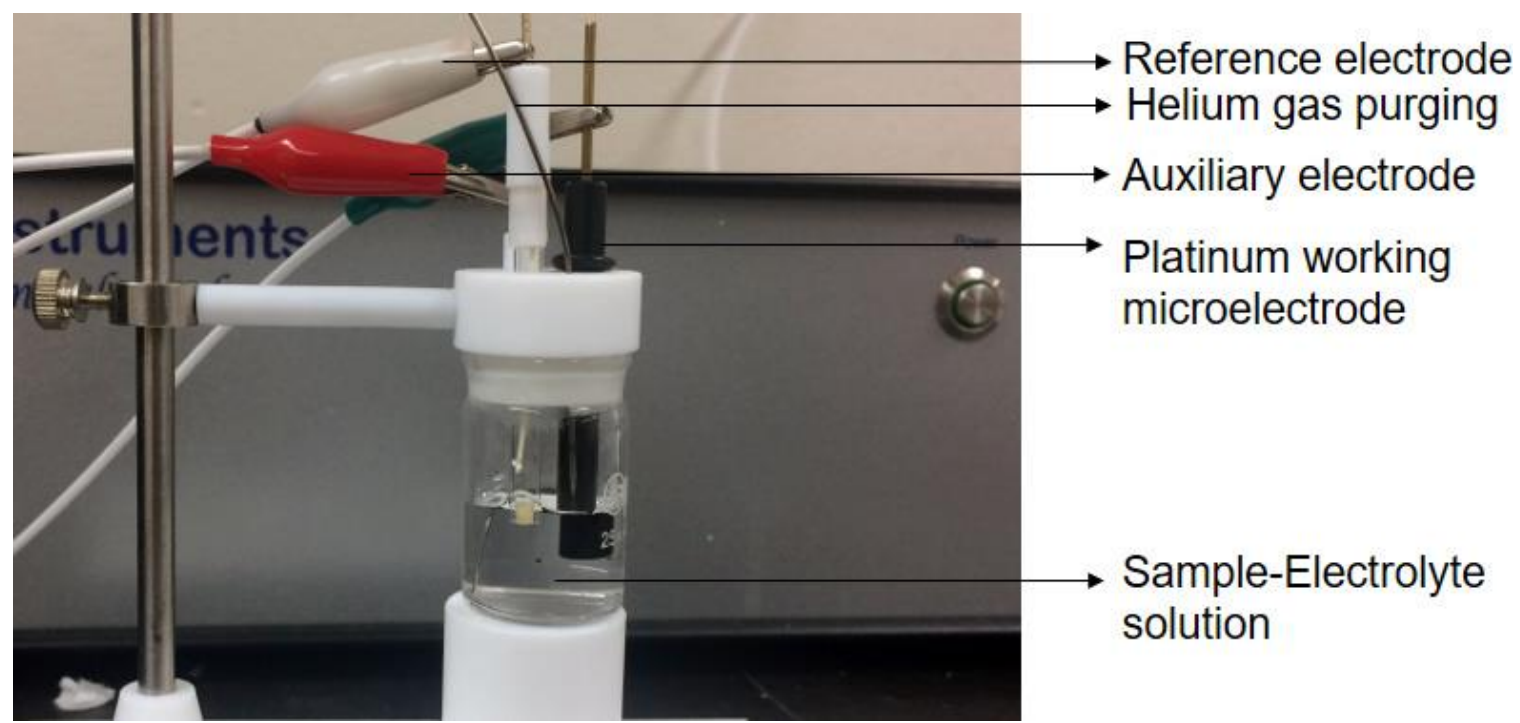

Figure 4.5 Experimental setup of liquid phase electrochemistry.

A different approach was performed to investigate the electrochemical behavior of the redox polymer. In this approach, the redox polymer (Fc-PEG) was deposited on the tip of the working electrode surface, which was wrapped with a polycarbonate membrane filter $(0.6 \mu \mathrm{m})$ using an O-ring. The working electrode wrapped with a polymer, reference and auxiliary electrodes were then dipped in the electrolyte solution for electrochemical measurements. Here the same electrodes were used for measurements as mentioned previously. The experimental setup for this approach is shown in Figure 4.6. 


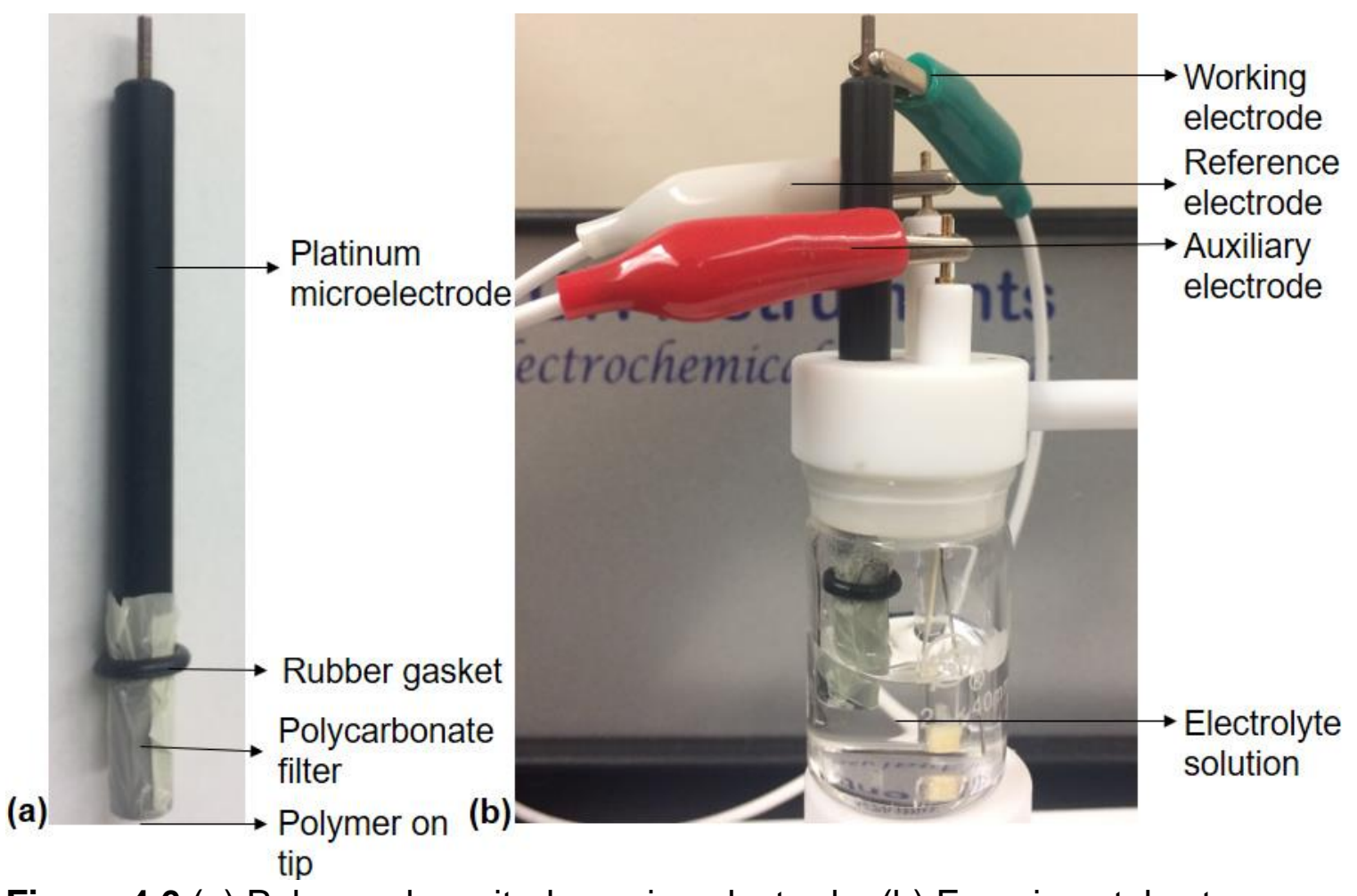

Figure 4.6 (a) Polymer deposited on microelectrode, (b) Experimental setup.

\subsubsection{Semi-Solid Phase Electrochemistry}

This method was used to analyze the redox additive polymer in semi-solid phase using the platinum ultramicroelectrode. The Fc-PEG polymer was mixed with few drops of an electrolyte and deposited on the ultramicroelectrode. A teflon block was made with a $3 \mathrm{~mm}$ hole through it, in which the platinum ultra microelectrode was inserted. This assembly was then placed on a silver plate which acted as reference and auxiliary electrodes. The coated ultramicroelectrode was then put through the teflon block for support and placed on a silver plate for electrical contact as shown in Figure 4.7. 


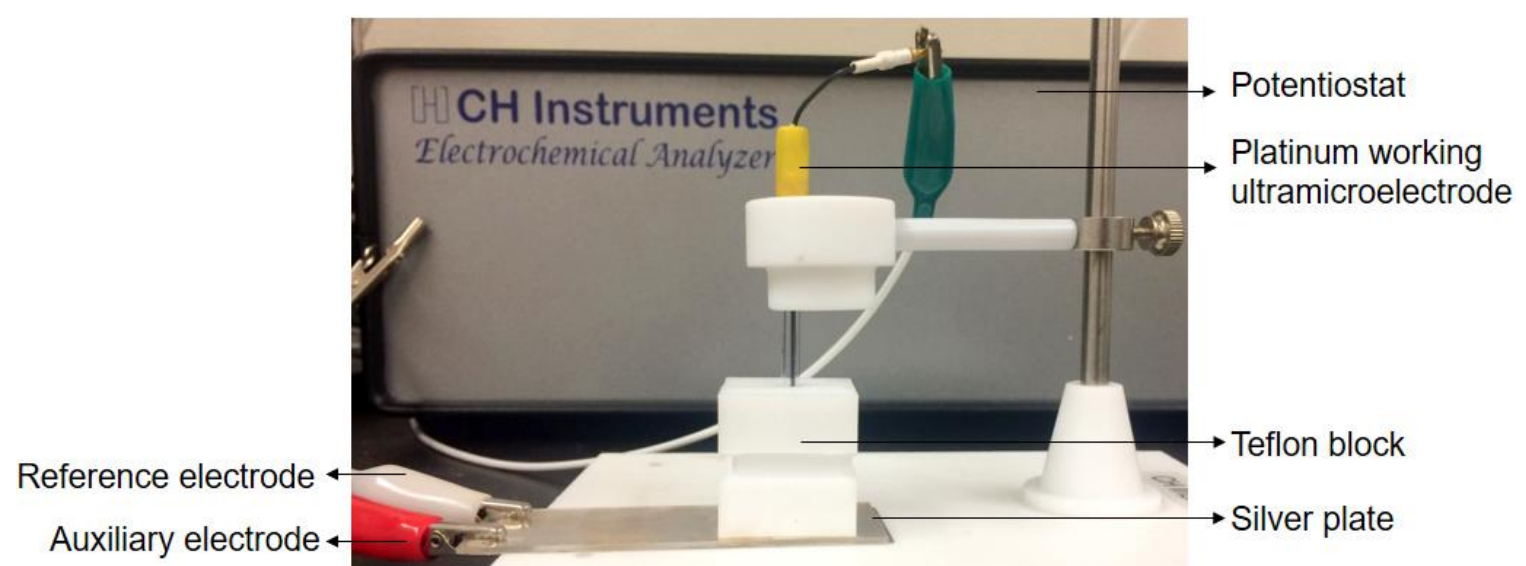

Figure 4.7 Experimental setup for semi-solid phase ultramicroelectrode voltammetry.

\subsection{Results and Discussion}

The following characterization techniques were used to confirm the product synthesized by the Steglish esterification with DIC reagent.

\subsubsection{NMR Spectroscopy}

The ${ }^{1} \mathrm{H}-\mathrm{NMR}$ and ${ }^{13} \mathrm{C}$-NMR spectra of PEG-ferrocene dicarboxylate polymer is shown in Figure 4.8 and 4.9 respectively. ${ }^{1} \mathrm{H}-\mathrm{NMR}(400 \mathrm{MHz}, \mathrm{DMSO}) \delta$ :

4.23,4.53 (s, 8H, cyclopentyl), 3.45 (m, 32H, $\left.-\mathrm{OCH}_{2}-\mathrm{CH}_{2}-\mathrm{O}-\right), 3.18(\mathrm{~s}, 2 \mathrm{H},-\mathrm{OH})$. ${ }^{13} \mathrm{C}-\mathrm{NMR}(400 \mathrm{MHz}, \mathrm{DMSO})$ ס: 72.96, 72.43, 71.71 (cyclopentyl), $70.42\left(-\mathrm{OCH}_{2}-\right.$ $\left.\mathrm{CH}_{2} \mathrm{O}-\right), 60.86$ (- $\left.\mathrm{COO}-\mathrm{CH}_{2} \mathrm{CH}_{2} \mathrm{O}-\right)$. The spectra was consistent with previously published work. ${ }^{56}$ The unmarked peaks are probably the impurities or byproducts from the reaction. 


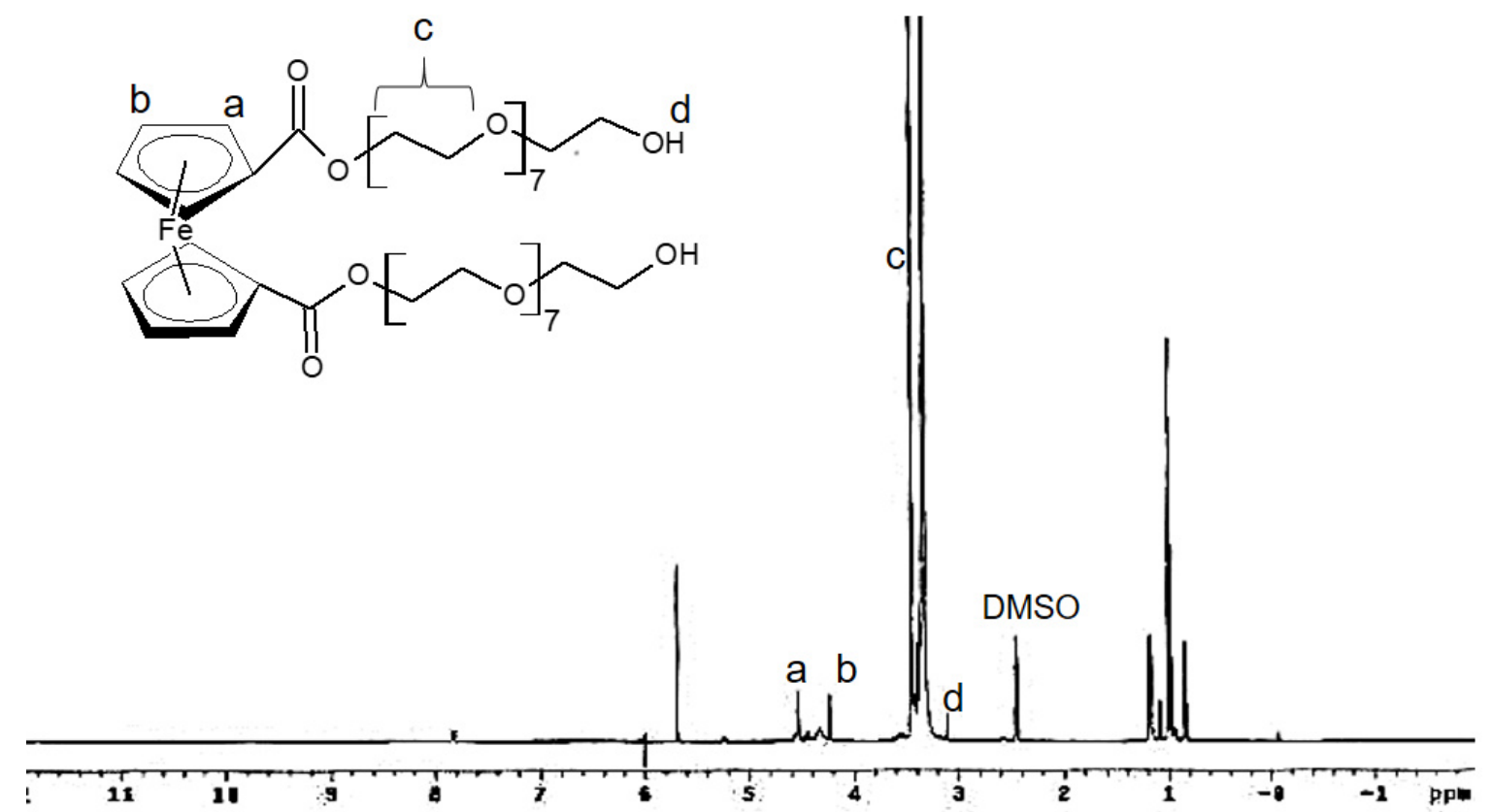

Figure 4.8 ${ }^{1} \mathrm{H}-\mathrm{NMR}$ spectrum of Fc-PEG polymer.

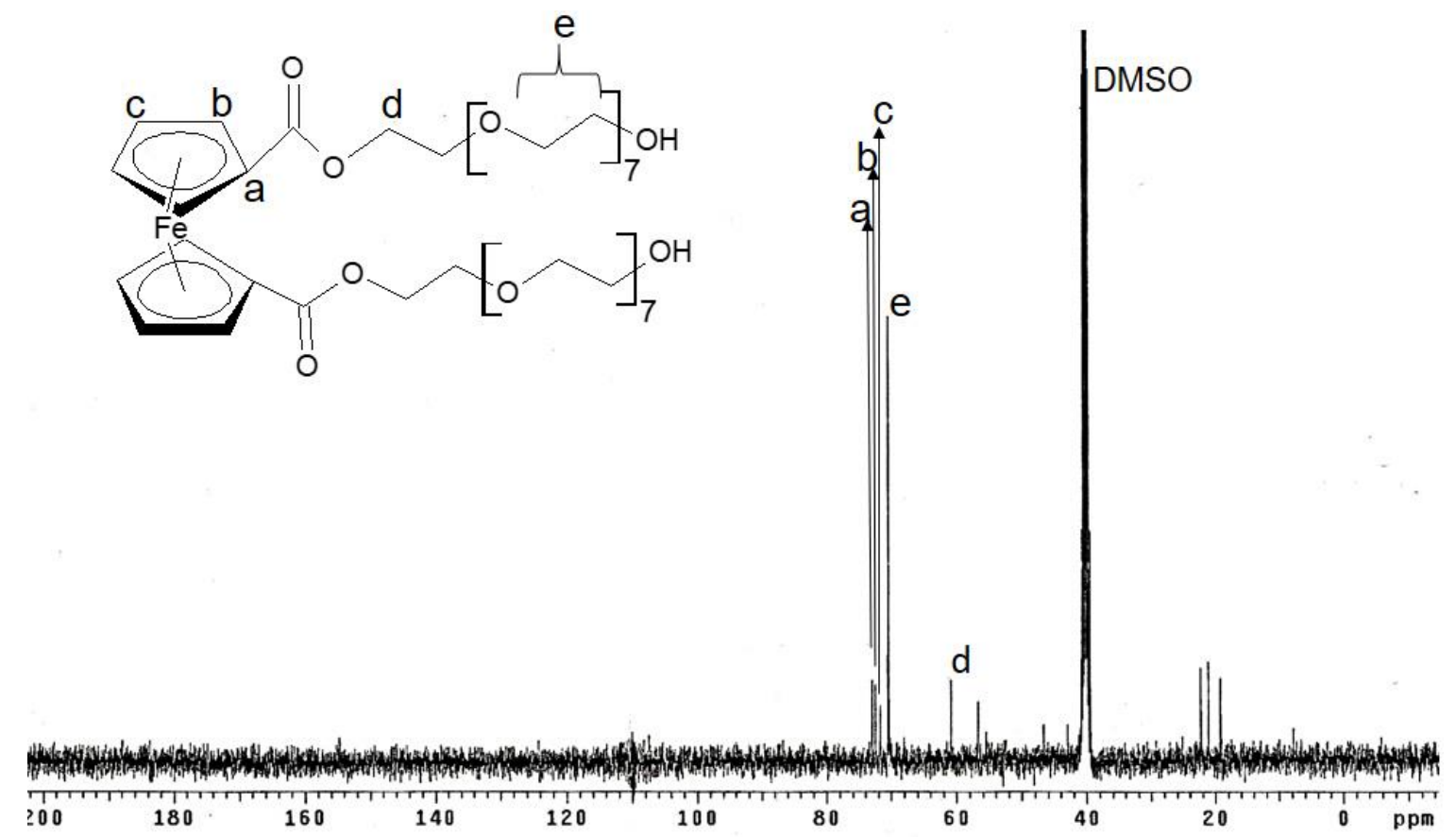

Figure $4.9{ }^{13} \mathrm{C}-\mathrm{NMR}$ spectrum of Fc-PEG polymer. 


\subsubsection{Electrochemical Analysis}

\subsubsection{Solution Phase Electrochemistry}

The cyclic voltammograms of ferrocene-dicarboxylic acid using a microelectrode and an ultramicroelectrode are shown in Figure 4.10 and Figure 4.11 respectively. For microelectrode, the potential was swept from -0.2 to $1.0 \mathrm{~V}$ at $0.1 \mathrm{~V} / \mathrm{s}$ scan rate. It can be seen from Figure 4.10 that the ferrocenedicarboxylic acid has one redox peak corresponding to the $\mathrm{Fc} / \mathrm{Fc}^{+}$redox couple with redox potential of $0.853 \mathrm{~V}$ and peak splitting $\Delta \mathrm{E}=93 \mathrm{mV}$, suggesting that it is reversible. For an ultramicroelectrode, the potential was swept from 0.6 to 1.2 $\mathrm{V}$ at a scan rate of $0.01 \mathrm{~V} / \mathrm{s}$. The shape of voltammogram was sigmoidal as shown in Figure 4.11 which is as expected for the ultramicroelectrodes.

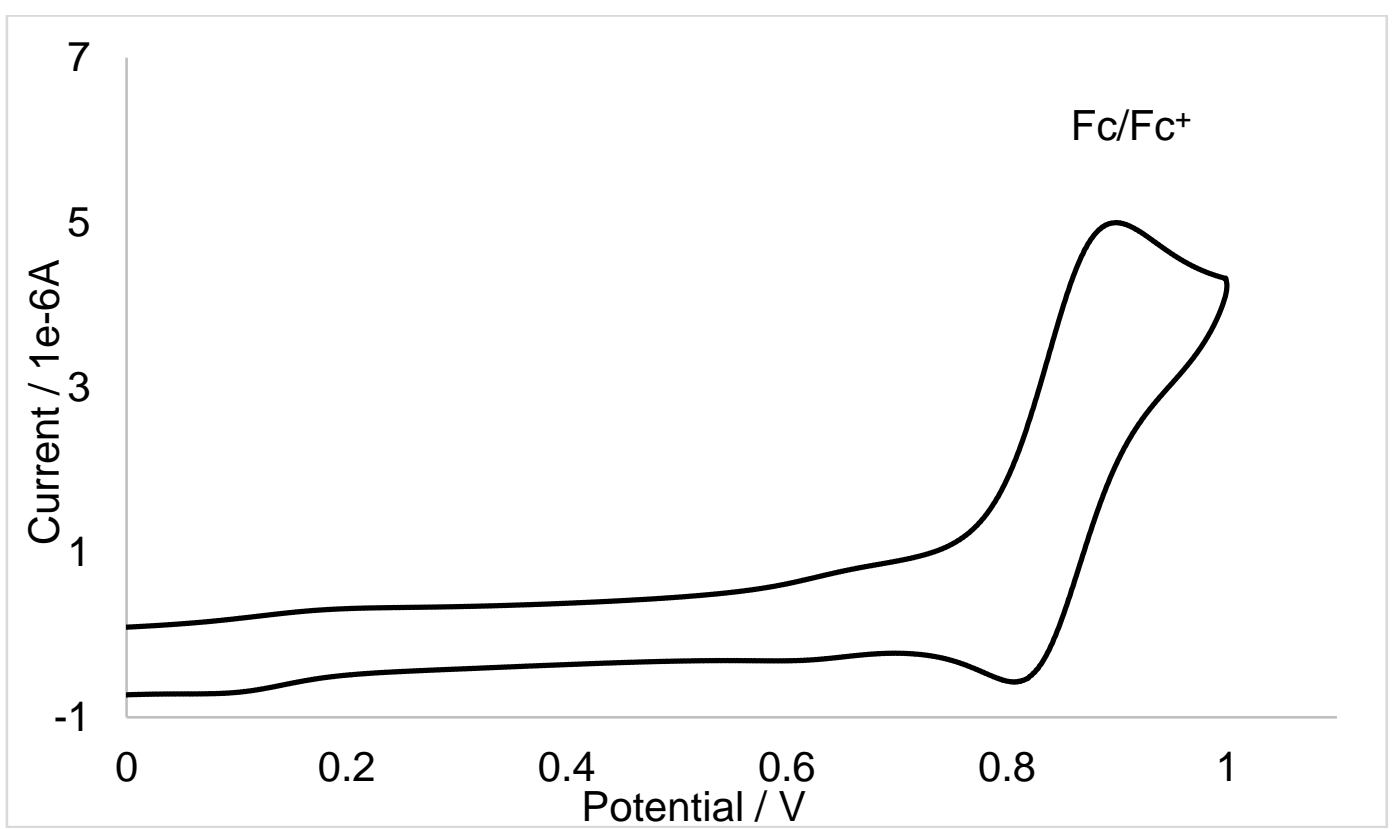

Figure 4.10 Cyclic Voltammogram of Ferrocene-dicarboxylic acid in acetonitrile with microelectrode. 


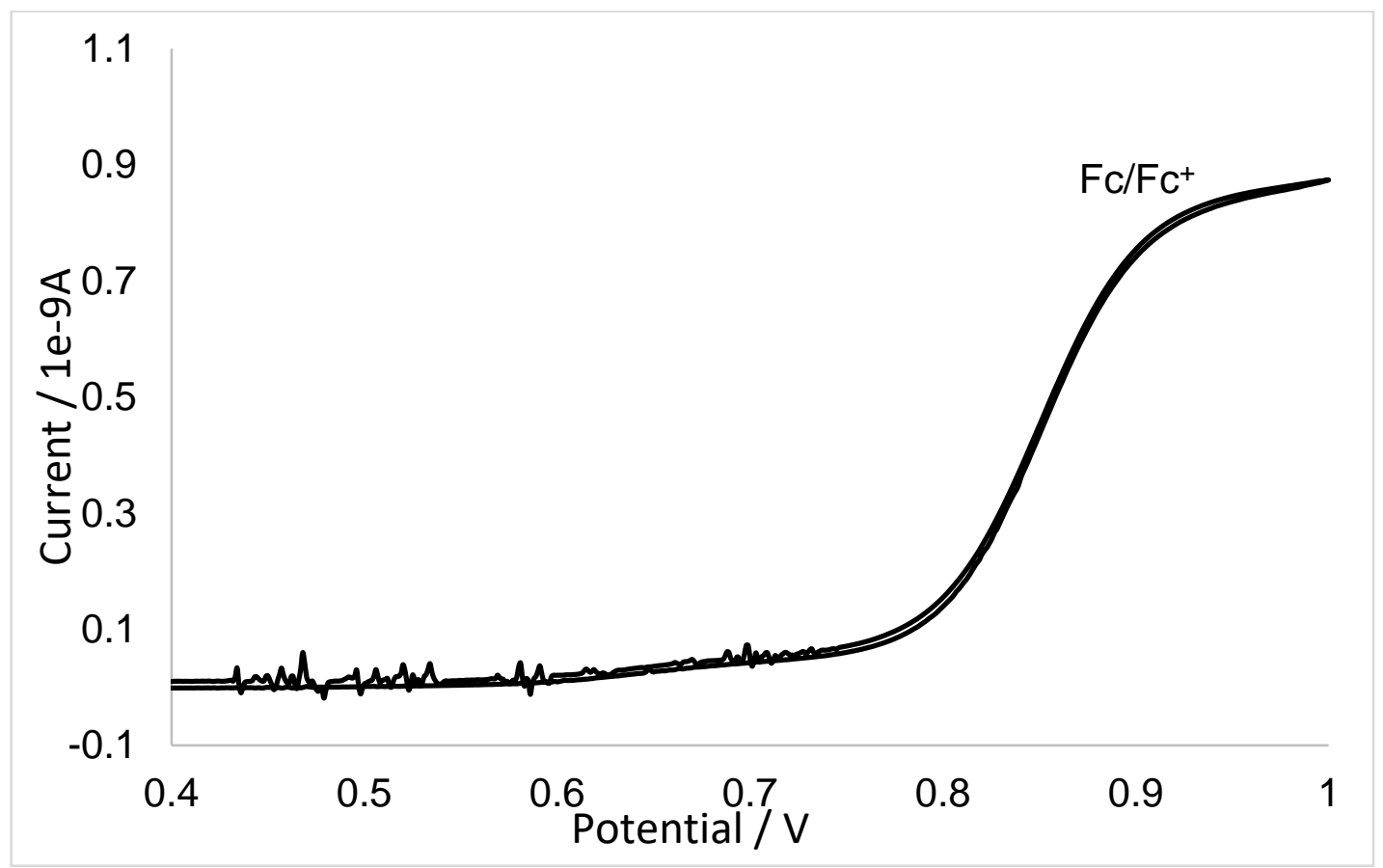

Figure 4.11 Cyclic Voltammogram of Ferrocene-dicarboxylic acid in acetonitrile with ultramicroelectrode.

The CVs of Fc-PEG polymer were recorded for both micro- and ultramicroelectrodes as shown in Figure 4.12 and 4.13 respectively. The potential was swept from -0.2 to $1.0 \mathrm{~V}$ at a scan rate of $0.1 \mathrm{~V} / \mathrm{s}$ for microelectrode. The $\mathrm{CV}$ graph showed one redox couple which corresponds to the $\mathrm{Fc} / \mathrm{Fc}^{+}$redox couple and is consistent with the formation of an ester linkage between PEG and ferrocene-dicarboxylic acid. The redox potential for polymer is $0.74 \mathrm{~V}$ and $\Delta \mathrm{E}=$ $95 \mathrm{mV}$ which is slightly shifted from ferrocene-dicarboxylic acid, as seen in Figure 4.12. This shift in the redox potential may be due to a quasi-reference electrode. For an ultramicroelectrode, the potential was swept from 0.0 to $1.0 \mathrm{~V}$ at $0.05 \mathrm{~V} / \mathrm{s}$. The CV was sigmoidal in shape as expected for an ultramicroelectrode (Figure 4.13). 


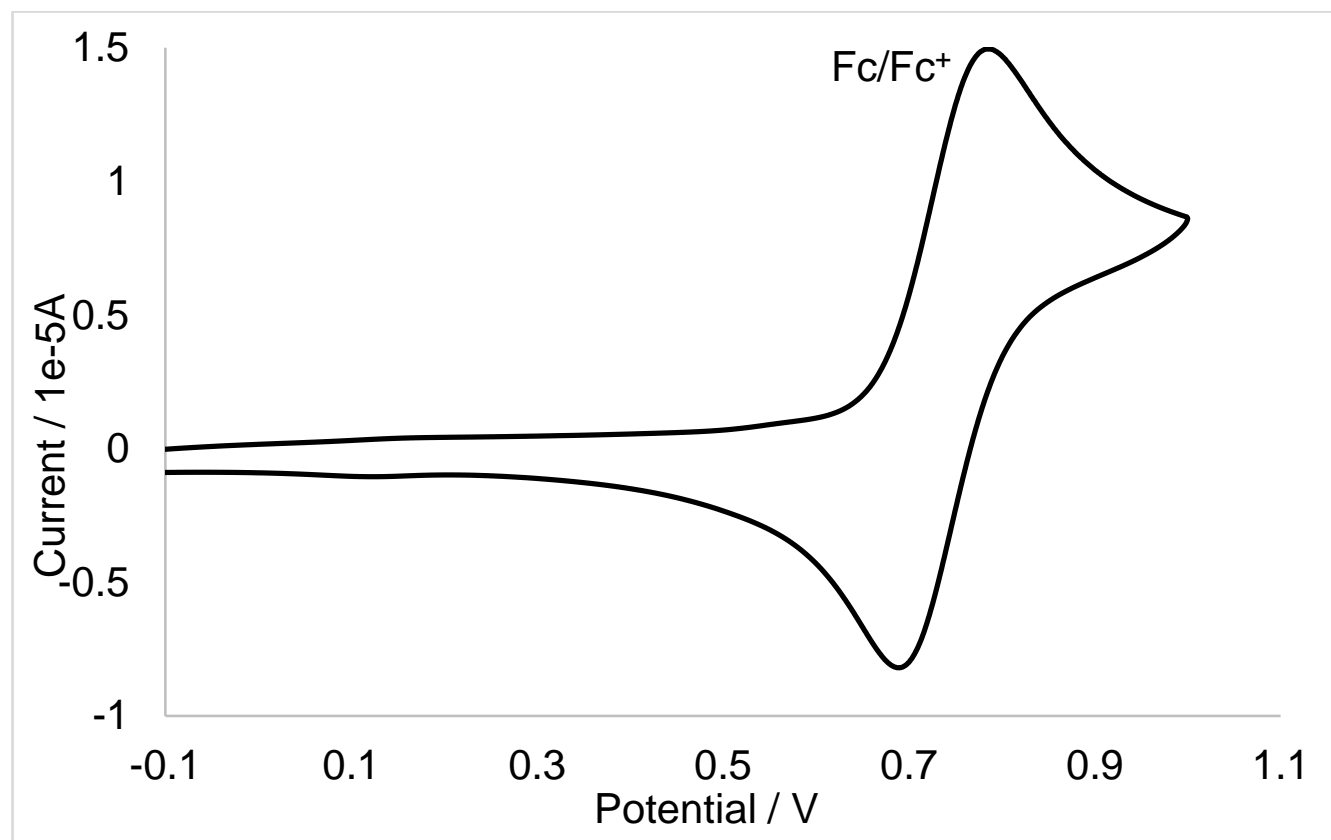

Figure 4.12 Cyclic Voltammogram of Fc-PEG polymer with microelectrode.

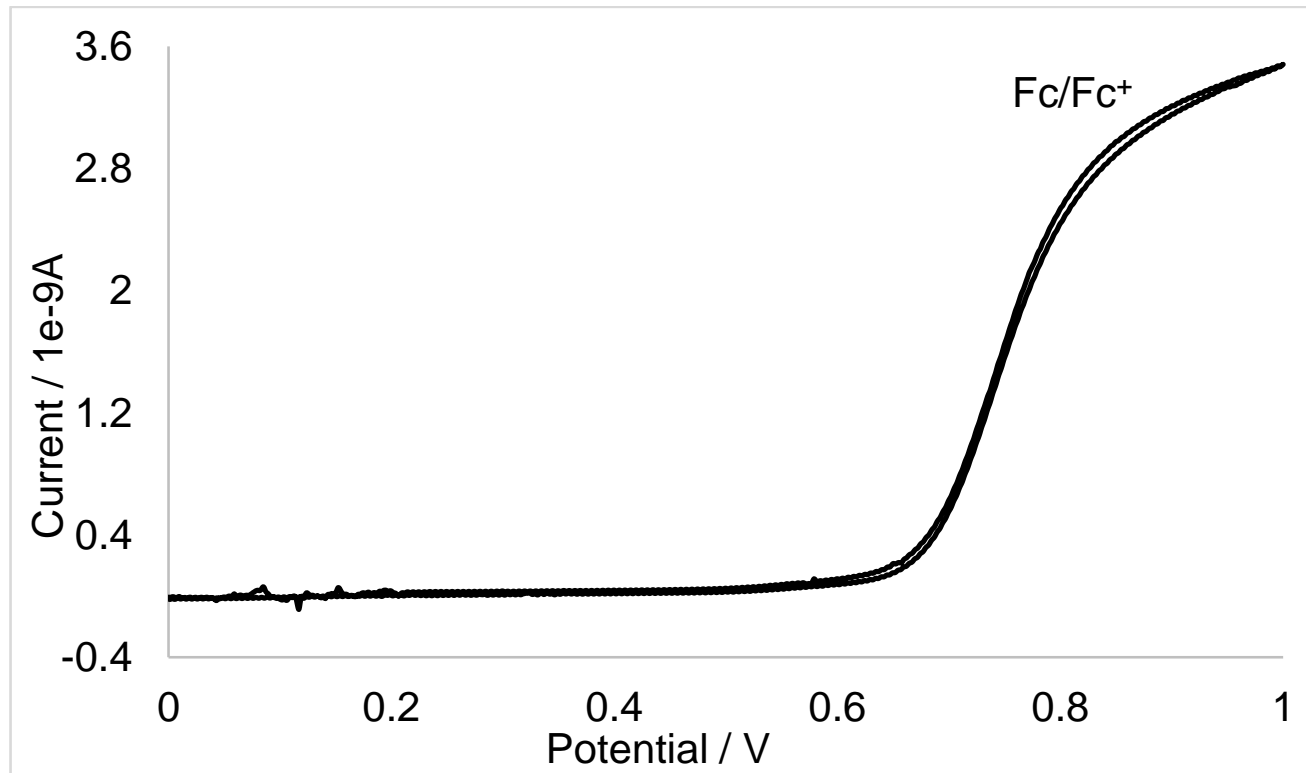

Figure 4.13 Cyclic Voltammogram of Fc-PEG polymer with ultramicroelectrode. 


\subsubsection{Semi-Solid Phase Electrochemistry}

The CV of Fc-PEG polymer was performed with an ultramicroelectrode as shown in Figure 4.14. The potential was swept between 0.5 to $1.2 \mathrm{~V}$ at $0.01 \mathrm{~V} / \mathrm{s}$ scan rate. The redox potential for the $\mathrm{Fc} / \mathrm{Fc}^{+}$redox couple is $0.89 \mathrm{~V}$ and $\Delta \mathrm{E}=$ $0.44 \mathrm{~V}$. The solid phase electrochemistry showed sigmoidal graph due to an ultramicroelectrode.

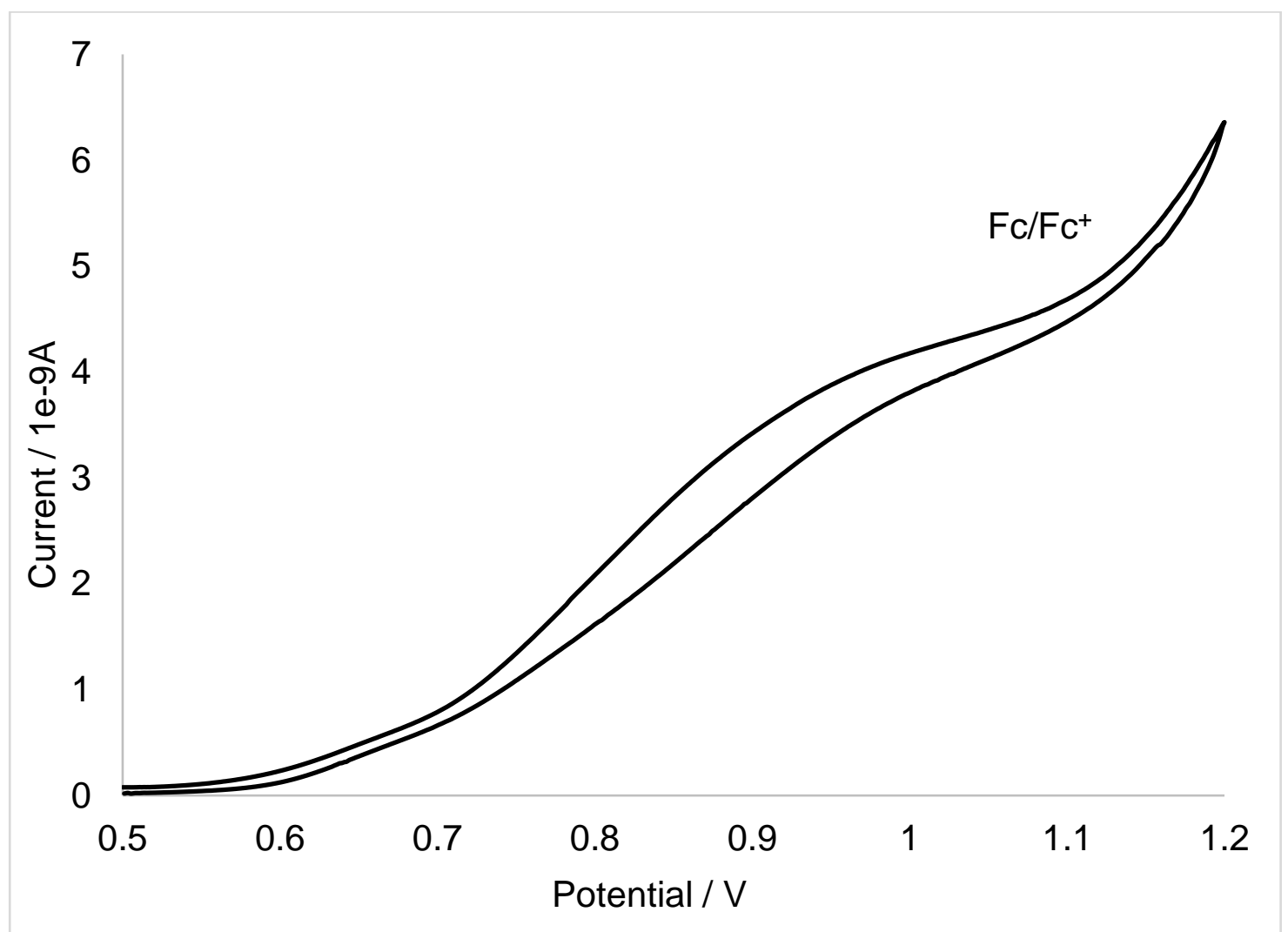

Figure 4.14 Cyclic Voltammogram of Fc-PEG polymer using silver plate.

\subsubsection{Fourier Transform Infrared Spectroscopy}

The stacked FTIR spectra of Fc-PEG polymer, ferrocene dicarboxylic acid, and PEG are shown in Figure 4.15 (a), (b), and (c), respectively. Sharp peaks at $1660 \mathrm{~cm}^{-1}$ and $1169 \mathrm{~cm}^{-1}$ corresponds to the $\mathrm{C}=\mathrm{O}$ and $\mathrm{C}-\mathrm{O}$ stretching modes 
respectively of the carboxylic acid groups of ferrocene-dicarboxylic acid (Figure 4.15 (b)). The broad peaks at $2645 \mathrm{~cm}^{-1}$ and $2980 \mathrm{~cm}^{-1}$ are related to the $\mathrm{O}-\mathrm{H}$ vibration of the carboxylic acid group and the $\mathrm{C}-\mathrm{H}$ stretching mode of the cyclopentyl rings of ferrocene. In the PEG spectrum (Figure 4.15 (c)), a sharp peak at $1098 \mathrm{~cm}^{-1}$ corresponds to the $\mathrm{C}$-O ether linkage and $2888 \mathrm{~cm}^{-1}$ peak is related to the $\mathrm{sp}^{3}$ hybridized carbon. The Fc-PEG polymer spectra (Figure 4.15 (a)) showed common peaks with starting compounds and an additional ester linkage absorption band. The peaks between $1600-1700 \mathrm{~cm}^{-1}$ and a band at $1180 \mathrm{~cm}^{-1}$ frequency corresponds to $\mathrm{C}=\mathrm{O}$ and $\mathrm{C}-\mathrm{O}$ ester linkage respectively between the PEG and ferrocene-dicarboxylic acid. The broad peak at $2985 \mathrm{~cm}^{-1}$ is related to the $\mathrm{C}-\mathrm{H}$ vibrational frequency of the cyclopentyl rings and absorption band at $2891 \mathrm{~cm}^{-1}$ corresponds to the $\mathrm{sp}^{3}$ hybridized $\mathrm{C}-\mathrm{H}$ bond of PEG chain. The other common peak is at $1060 \mathrm{~cm}^{-1}$ corresponds to the C-O ether bond of PEG. Therefore, the ester linkage absorption band in the Fc-PEG polymer spectrum confirms the covalent attachment of PEG and ferrocene-dicarboxylic acid. 


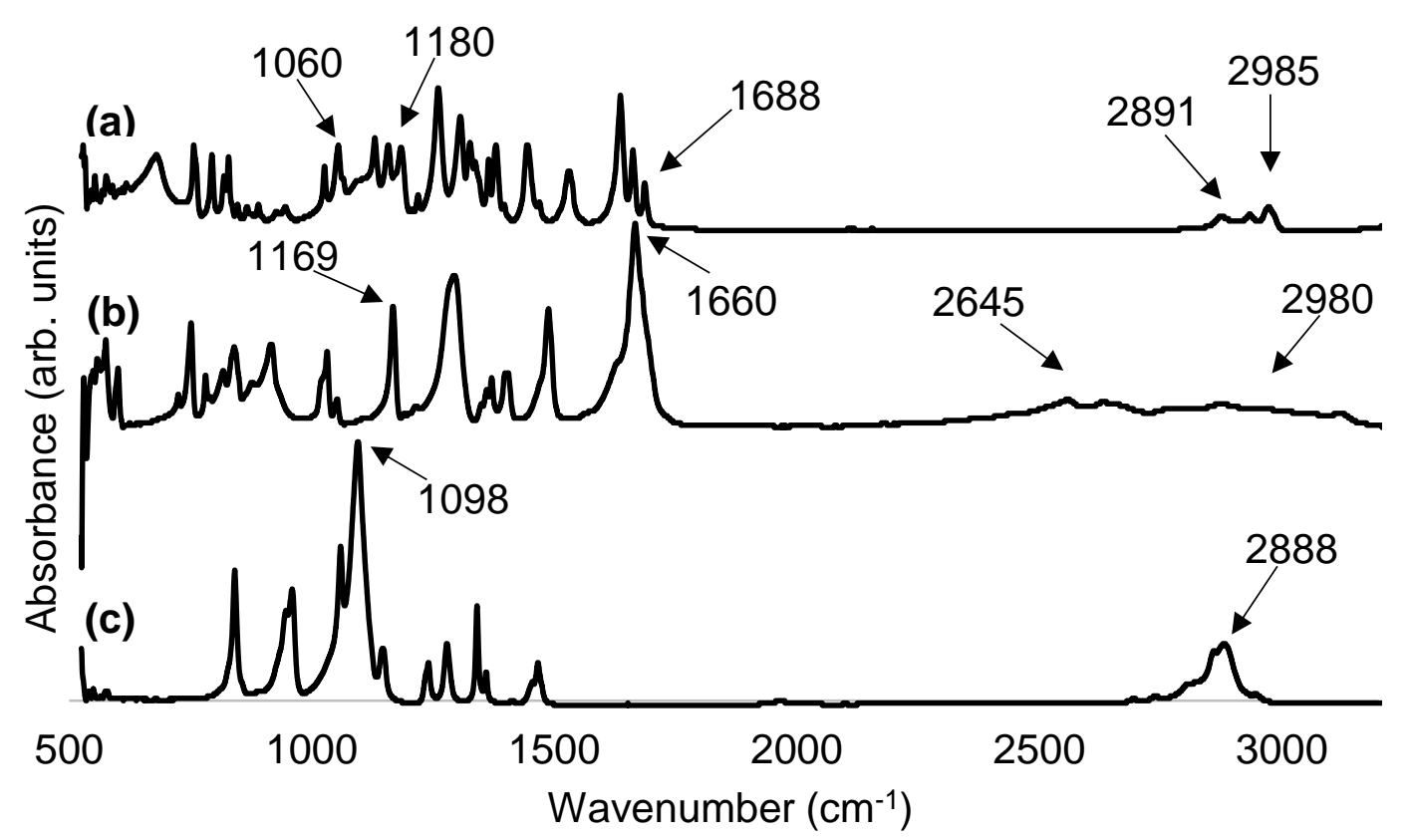

Figure 4.15 FTIR spectra of (a) Fc-PEG polymer, (b) ferrocene-dicarboxylic acid, and (c) PEG.

\subsection{Conclusions}

The Steglish esterification method using the DIC reagent proved to be the best method for the synthesis of Fc-PEG polymer. Both ${ }^{1} \mathrm{H}$ and ${ }^{13} \mathrm{C}$ NMR spectra showed an ester linkage peak. The $\mathrm{CV}$ of the $\mathrm{Fc}-\mathrm{PEG}$ polymer resulted in $\mathrm{Fc} / \mathrm{Fc}^{+}$ redox peaks which confirmed the covalent attachment of PEG and ferrocenedicarboxylic acid as PEG is electrochemically inactive. The FTIR spectrum also showed ester absorption peaks at $1688 \mathrm{~cm}^{-1}$ and $1188 \mathrm{~cm}^{-1}$ corresponding to $\mathrm{C}=\mathrm{O}$ and $\mathrm{C}-\mathrm{O}$ stretch modes which confirmed the ester formation. This Fc-PEG polymer will act as polyelectrolyte in the cell and will be grafted to functionalized SWCNTs which is discussed in Chapter 5. 


\section{Chapter 5 : GRAFTING OF CARBON NANOTUBES WITH FERROCENE- POLYEHTYLENE GLYCOL POLYMER}

\subsection{Introduction}

The grafting of nanotubes to the Fc-PEG polymer increases the solvent accessible surface area of SWCNTs, and thus increases the ion mobility from the electrolyte to the current collector as discussed in Chapter 2. The SWCNTFerrocene (Fc) polymer was synthesized in two steps: the first step was to activate refluxed nanotubes (mentioned in Chapter 3) using acyl chlorination and the second step was to react Fc-PEG polymer (synthesized in Chapter 4) with the activated nanotubes. The reaction is shown in Scheme 5.1 and the mechanism for the acyl chlorination is discussed in detail in Chapter 4.

Scheme 5.1 Scheme of (a) acyl chlorination of SWCNTs and (b) formation of SWCNT-Fc polymer composite.
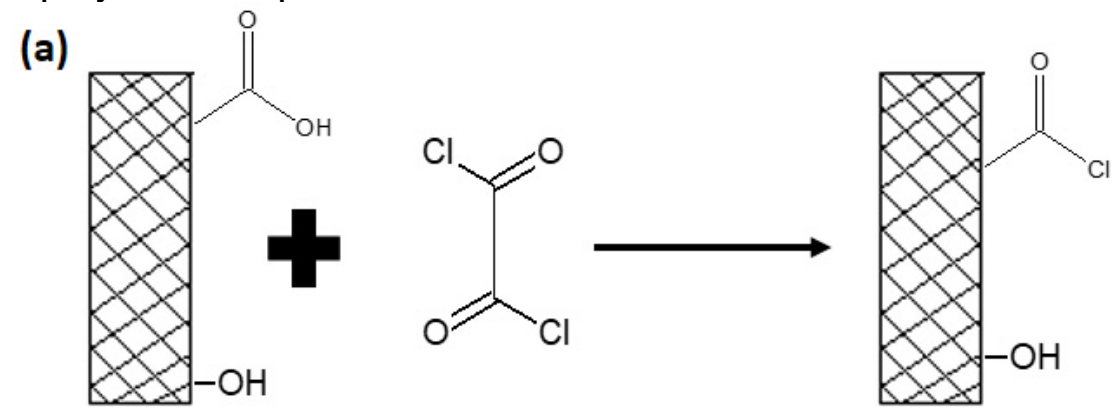

(b)

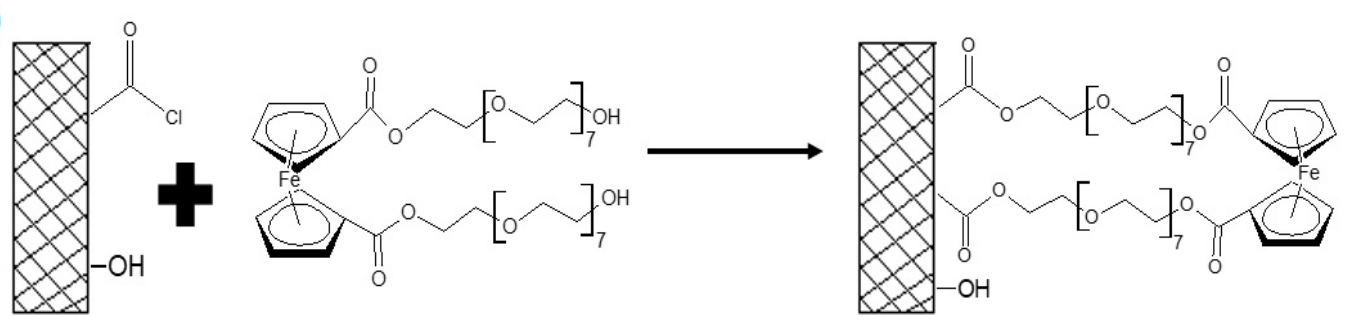




\subsection{Materials}

Acetonitrile, dimethyl formamide (DMF) and oxalyl chloride were obtained from Sigma Aldrich Corporation and were used as received. All liquid solvents and solid reactants were dried using molecular sieves and stored in the refrigerator. Glass syringes were used to transfer dried liquid reagents and solvents into the reaction mixture. All reactions were performed in a three-neck round bottom flask fitted with a rubber septum under an inert nitrogen atmosphere.

\subsection{Experimental Methods}

Refluxed SWCNTs (50 mg), were sonicated in $20 \mathrm{~mL}$ DMF for 30 minutes to make a homogenous suspension. This solution was then stirred for 30 minutes in an ice bath. The oxalyl chloride $(4 \mathrm{~mL})$ was added dropwise to the resultant suspension and stirred for 2 hours in an ice bath. After the oxalyl chloride addition, the reaction mixture was stirred for an hour at room temperature and was then increased to $70^{\circ} \mathrm{C}$ for 8 hours to remove any unreacted oxalyl chloride.

FC-PEG polymer (0.08 mmol, $50 \mathrm{mg}$ ) was dissolved in $5 \mathrm{~mL}$ DMF and added to the above reaction mixture. The reaction mixture was stirred for 5 days at $100^{\circ} \mathrm{C}$. It was then cooled to room temperature and the solvent was evaporated to dryness, to obtain the resulting black color grafted SWCNT- ferrocene (Fc) polymer. The product was then dried under vacuum and used without further purification. ${ }^{65,66,67}$ 


\subsection{Characterization Techniques}

FTIR is one of the few analytical techniques suitable for the identification of organic compounds in these materials. The molecule responsible for IR absorption vibrates at a frequency characteristic of the functional groups, e.g. amides, esters, carbonyls, etc. The FTIR spectrum of SWCNT-Fc polymer was recorded on the Thermo Nicolet 6700 FT-IR spectrometer.

Electrochemical analysis was done on $\mathrm{CH} 660$ Electrochemical Analyzer, and cyclic voltammetry (CV) was used to investigate the redox behavior of SWCNTFc polymer.

\subsection{Results and Discussion}

\subsubsection{Fourier Transform Infrared Spectroscopy}

The stacked FTIR spectra of SWCNT-Fc polymer, refluxed SWCNTs, and FcPEG polymer in an inset are shown in Figure 5.1. The absorption band at $2150 \mathrm{~cm}^{-1}$ corresponds to the $\mathrm{sp}^{2}$ hybridized carbon stretching mode of carbon nanotubes in SWCNT-Fc polymer (Figure 5.1 (a)). This vibrational frequency coincides with that of the refluxed SWCNTs. The additional sharp peaks in the $600-1700 \mathrm{~cm}^{-1}$ and $1150-1173 \mathrm{~cm}^{-1}$ corresponds to the $\mathrm{C}=\mathrm{O}$ and $\mathrm{C}-\mathrm{O}$ stretching modes respectively. These groups are from the ester linkage between Fc-PEG and SWCNTs, and within the Fc-PEG. The other prominent bands seen in Figure 5.1 (a) are common with the Fc-PEG polymer, at $2766 \mathrm{~cm}^{-1}$ corresponding to the $\mathrm{C}-\mathrm{H}$ alkane stretching mode of the PEG chain, and at about $2960 \mathrm{~cm}^{-1}$ related to the $\mathrm{C}-\mathrm{H}$ alkene stretching mode of the cyclopentyl ferrocene 
rings as discussed in Chapter 4. The SWCNT-Fc polymer spectra can be compared with the FTIR spectra of Fc-PEG polymer, shown in an inset of Figure 5.1. The FTIR spectra of the SWCNT-Fc polymer was not conclusive because of the presence of multiple ester groups. Therefore, the FTIR spectra did not confirm the covalent attachment of Fc-PEG polymer and SWCNTs. In contrast, the common absorption bands confirmed the formation of SWCNTs and polymer composite.

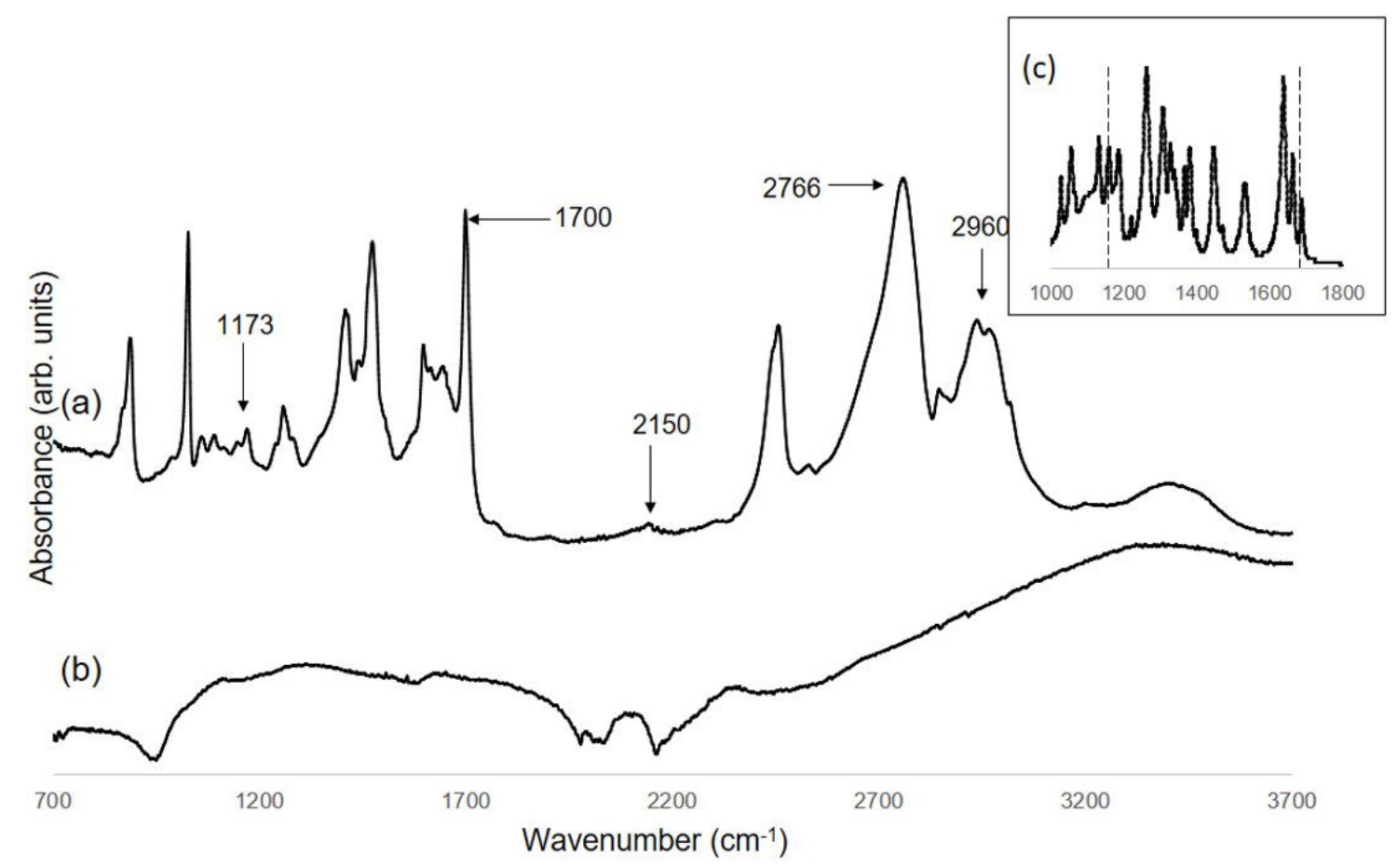

Figure 5.1 FTIR spectra of (a) SWCNT-Fc polymer and (b) refluxed SWCNTs. Inset (c) shows the FTIR spectrum of Fc-PEG polymer.

\subsubsection{Electrochemical Analysis}

The cyclic voltammogram of the SWCNT-Fc polymer was recorded using a semi-solid phase ultramicroelectrode voltammetry as discussed in Chapter 4. In this cell, the silver plate acted as a reference and an auxiliary electrode and a 10 
$\mu \mathrm{m}$ platinum ultramicroelectrode was used as the working electrode. The potential was swept between 0 to $0.7 \mathrm{~V}$ with a scan rate of $0.03 \mathrm{~V} / \mathrm{s}$. Figure 5.2 shows the cyclic voltammogram exhibiting a plateau corresponding to the $\mathrm{Fc} / \mathrm{Fc}^{+}$ redox couple at $0.37 \mathrm{~V}$. This general peak shape corresponds clearly to the effective hemispherical diffusion and attests to the good charge transfer rates in this matrix of SWCNTs and polymer. The apparent diffusion coefficient of $\mathrm{Fc} / \mathrm{Fc}^{+}$ assuming a $1 \mathrm{M}$ concentration of redox sites was $3.0 \times 10^{-8} \mathrm{~cm}^{2} / \mathrm{s}$, which was calculated using a radial diffusion equation discussed in Chapter 4 (Equation 4.3). The shape of the graph is broad because of capacitance due to nanotubes.

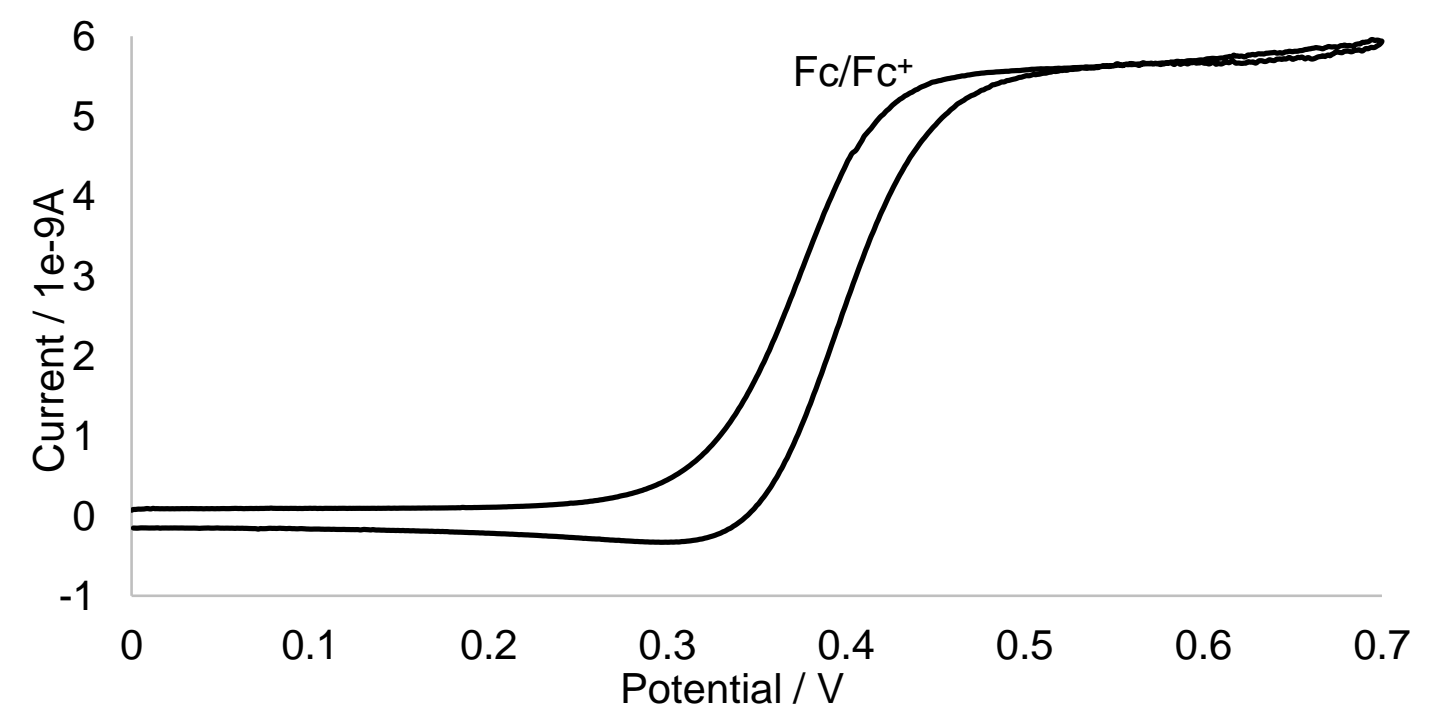

Figure 5.2 Cyclic Voltammogram of SWCNT-Fc polymer.

\subsection{Conclusions}

The $\mathrm{CV}$ showed reversible $\mathrm{Fc} / \mathrm{Fc}^{+}$redox peaks which indicated the esterification between Fc-PEG and SWCNT was in part successful, despite the ambiguity of the FTIR spectra. FTIR spectra showed common absorption peaks at $2766 \mathrm{~cm}^{-1}$ and $2960 \mathrm{~cm}^{-1}$ corresponding to the Fc-PEG polymer. However, the 
frequencies corresponding to the ester linkage were not conclusive in confirming the covalent linkage between SWCNTs and Fc-PEG polymer because of the large number of ester groups present in the polymer backbone. This SWCNT-FC polymer composite was further used in the fabrication of the hybrid cell discussed in the next chapter. 


\section{Chapter 6 : FABRICATION OF PROTOTYPE CELL}

\subsection{Background}

The prototype hybrid supercapacitor cell is fabricated using the SWCNT-FC polymer (discussed in Chapter 5), which acts as a reduction half-cell and the SWCNT-viologen polymer that was synthesized by laboratory colleague Kanishka Rana ${ }^{27}$ was used as an oxidation half-cell. When a potential is applied, the ferrocene species gets oxidized first to ferricenium $\left(\mathrm{Fe}^{+3}\right)$ ion and electrons flow from the positive to the negative electrode via an external circuit because of a higher redox potential than the viologen species. The electrons at the negative electrode, reduce the $\mathrm{V}^{+2}$ to $\mathrm{V}^{+}$. Along with this, there is also formation of an electric double layer at the interface between SWCNTs and electrolyte at both electrodes. The perchlorate ions in the electrolyte flow towards the positive electrode via a separator, whereas lithium ions flow towards the negative electrode. Once all of the electrons and ions are transferred, the cell is fully charged and ready to use.

During discharging, the ions flow towards the opposite electrode and electrons flow from the negative to the positive electrode through the outer circuit. Once all the ferricenium ions are reduced to ferrocene and $\mathrm{V}^{+}$is oxidized to $\mathrm{V}^{2+}$, the battery is fully discharged and needs re-charging. The redox reaction contributes to the faradaic capacitance and the electrical double layer contributes to the non-faradaic capacitance. The faradaic and non-faradaic processes are expected to increase the energy density of the hybrid cell. The mechanism of the 
hybrid cell is illustrated in Figure 6.1. The redox reactions during charging and discharging at both the positive and negative electrodes are depicted below from equations $6.1-6.4$.

During charging:

At positive electrode:

$$
\begin{aligned}
\mathrm{E}_{\mathrm{X}}+\mathrm{A}^{-} & \rightarrow \mathrm{E}_{\mathrm{X}}^{+} / / \mathrm{A}^{-}+\mathrm{e}^{-} \\
\mathrm{Fc}+\mathrm{ClO}_{4}^{-} & \rightarrow \mathrm{Fc}^{+} / / \mathrm{ClO}_{4}^{-}+\mathrm{e}^{-} \quad \text { Equation } 6.1
\end{aligned}
$$

At negative electrode:

$$
\begin{gathered}
\mathrm{E}_{\mathrm{y}}+\mathrm{C}^{+}+\mathrm{e}^{-} \rightarrow \mathrm{E}_{\mathrm{y}}^{-} / / \mathrm{C}^{+} \\
\mathrm{V}^{2+}+\mathrm{Li}^{+}+\mathrm{e}^{-} \rightarrow \mathrm{V}^{+} / / \mathrm{Li}^{+}
\end{gathered}
$$

During discharging:

At positive electrode:

$$
\begin{gathered}
\mathrm{E}_{\mathrm{X}}^{+} / / \mathrm{A}^{-}+\mathrm{e}^{-} \rightarrow \mathrm{E}_{\mathrm{X}}+\mathrm{A}^{-} \\
\mathrm{Fc}^{+} / / \mathrm{ClO}_{4}^{-}+\mathrm{e}^{-} \rightarrow \mathrm{Fc}+\mathrm{ClO}_{4}^{-}
\end{gathered}
$$

At negative electrode:

$$
\begin{aligned}
& \mathrm{E}_{\mathrm{y}}^{-} / / \mathrm{C}^{+} \rightarrow \mathrm{E}_{\mathrm{y}}+\mathrm{C}^{+}+\mathrm{e}^{-} \\
& \mathrm{V}^{+} / / \mathrm{Li}^{+} \rightarrow \mathrm{V}^{2+}+\mathrm{Li}^{+}+\mathrm{e}^{-}
\end{aligned}
$$

where $E_{x}$ is the SWCNT-Fc electrode, $E_{y}$ is the SWCNT-V electrode, $A^{-}$is the perchlorate ion, $\mathrm{C}^{+}$is the lithium ion and//represents the interface of electrode and electrolyte. 


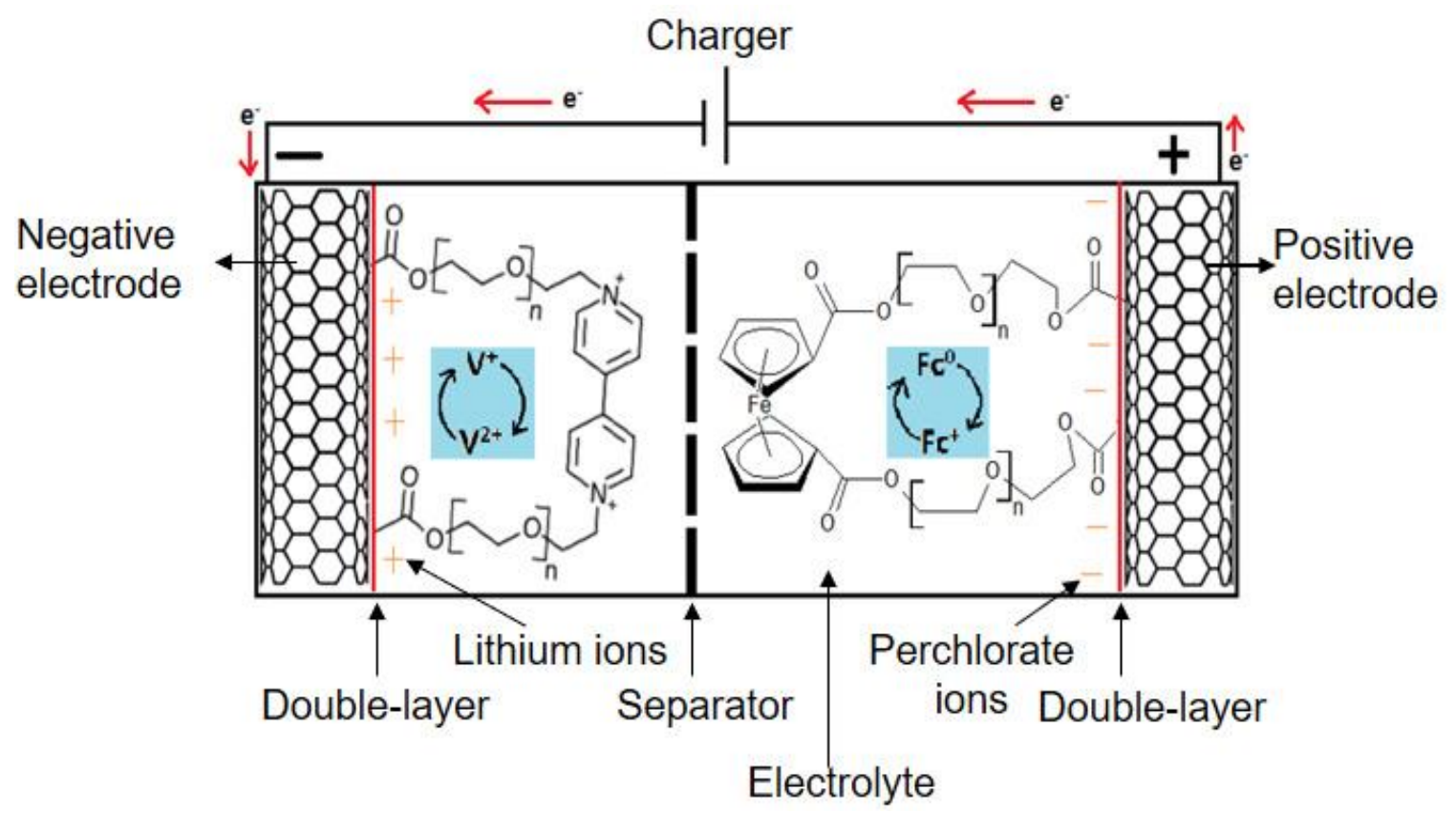

Figure 6.1 Mechanism of a hybrid supercapacitor cell during charging.

The electrochemical performance of the cell was analyzed by cyclic voltammetry $(\mathrm{CV})$ and chronoamperometry $(\mathrm{CA})$ techniques. $\mathrm{CV}$ is used to investigate the electrochemical behavior of the analytes which can be electrochemically oxidized or reduced. This technique is explained in detail in Chapter 4. CA is another electrochemical technique which investigates the kinetics of chemical reactions and diffusion processes. The current is measured as a function of time with response to the applied step potential. The recorded current can be of two types depending on the run time of the experiment: for a short time scale, the capacitive current is dominant while the faradaic current is dominant for a longer time. Initially, the potential of the working electrode is held at $E_{i}$ and at $t=0$, then it is changed instantaneously to a new value $E_{1}$ (Figure 6.2 
(a)). The corresponding current vs time response is recorded as shown in Figure $6.2(b) \cdot 68,69$

(a)

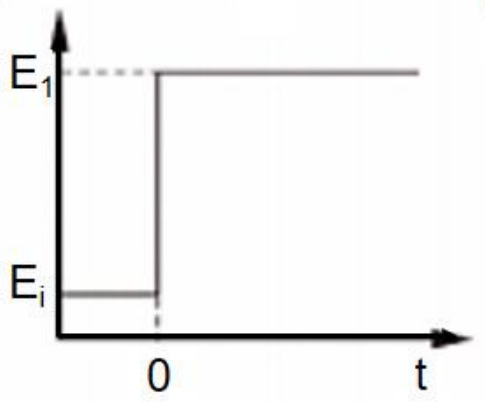

(b)

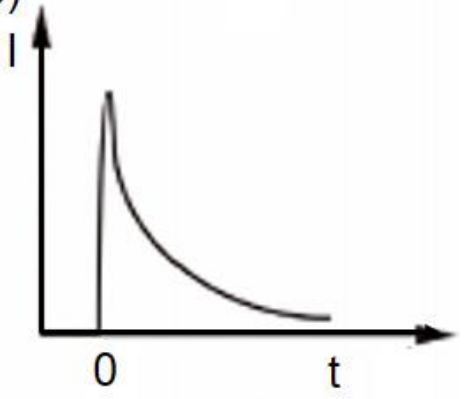

Figure 6.2 The chronoamperometric experiment. (a) The potential-time profile applied during experiment, $E_{i}$ is initial value and $E_{1}$ is the final value. (b) The corresponding response of the current due to changes of the potential.

For the diffusion-controlled or faradaic process, the current follows the Cottrell equation as shown:

$$
i=\frac{n F A c_{j}^{0} \sqrt{ } D_{j}}{\sqrt{ } \pi t}
$$

where $\mathrm{i}$ is the current in amperes, $\mathrm{n}$ is the number of electrons in the redox reaction, $\mathrm{F}$ is the Faraday constant, $\mathrm{A}$ is the area of the electrode in $\mathrm{cm}^{2}, \mathrm{c}_{\mathrm{j}}^{0}$ is the initial concentration of analyte $\mathrm{j}$ in $\mathrm{mol} / \mathrm{cm}^{3}, D_{j}$ is the diffusion coefficient for species $\mathrm{j}$ in $\mathrm{cm}^{2} / \mathrm{s}$, and $\mathrm{t}$ is the time in $\mathrm{s} .{ }^{69}$

The current is largely non-faradaic at short time scales due to the charging of the double-layer capacitance. The non-faradaic current decays exponentially with time constant $\mathrm{RC}$ as shown:

$$
i=\frac{E}{R} e^{-t / R C}
$$


where $\mathrm{E}$ is the potential applied, $\mathrm{R}$ is the resistance, and $\mathrm{C}$ is the double-layer capacitance. ${ }^{69}$

\subsection{Cell Fabrication Procedure}

The materials used to assemble the hybrid supercapacitor cell are aluminum C-clamp, aluminum current collectors, platinum foils, silicone gaskets, SWCNTFc composite, viologen grafted SWCNTs, and a polycarbonate membrane separator. The electrolyte used was $0.1 \mathrm{M}$ lithium perchlorate in dry acetonitrile.

In this work, the current collectors were polished to remove any impurities and platinum foil was placed at the center. Silicone gaskets were pasted over aluminum current collectors to prevent short circuiting and with platinum cavity for sample deposition. This configuration is illustrated in Figure 6.3.

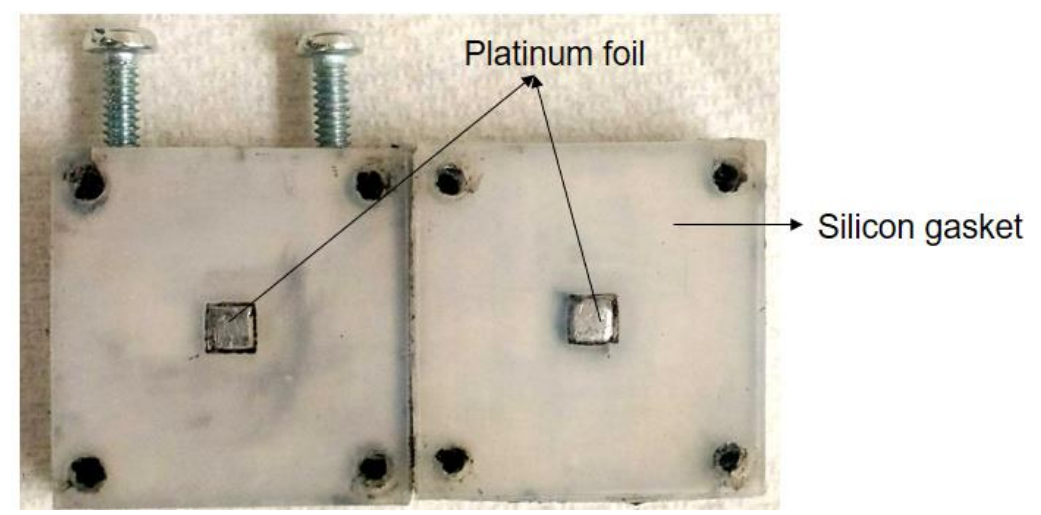

Figure 6.3 Aluminum current collectors with silicon gaskets and platinum foils.

The paste of the SWCNT-Fc composite $(3 \mathrm{mg}$ ) with an electrolyte was deposited on the platinum which acted as the working electrode and the viologen grafted SWCNTs (3 mg) and electrolyte paste was deposited on the other platinum foil which was used as the reference and auxiliary electrode. Once the sample was deposited, 19 polycarbonate separator membranes were placed in 
between the two electrodes and were clamped together with a C-clamp in sandwich model configuration as shown in Figure 6.4. While clamping a Teflon sheet was placed on the reference electrode side to avoid short circuiting of the cell. The configuration of the cell for experiments is illustrated in Figure 6.5. In all the CV and CA experiments, the configuration of the cell was kept the same.

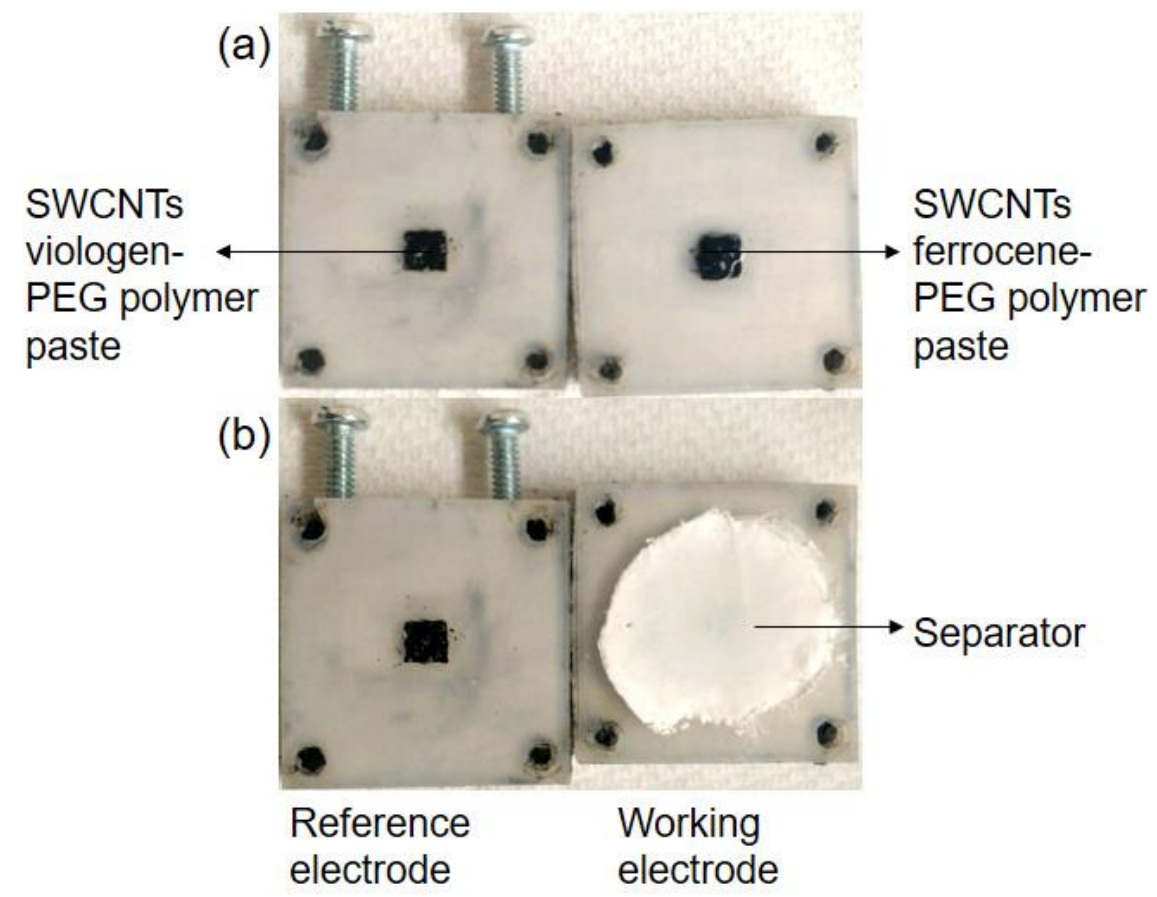

Figure 6.4 Aluminum current collectors with (a) analyte and (b) separator. 


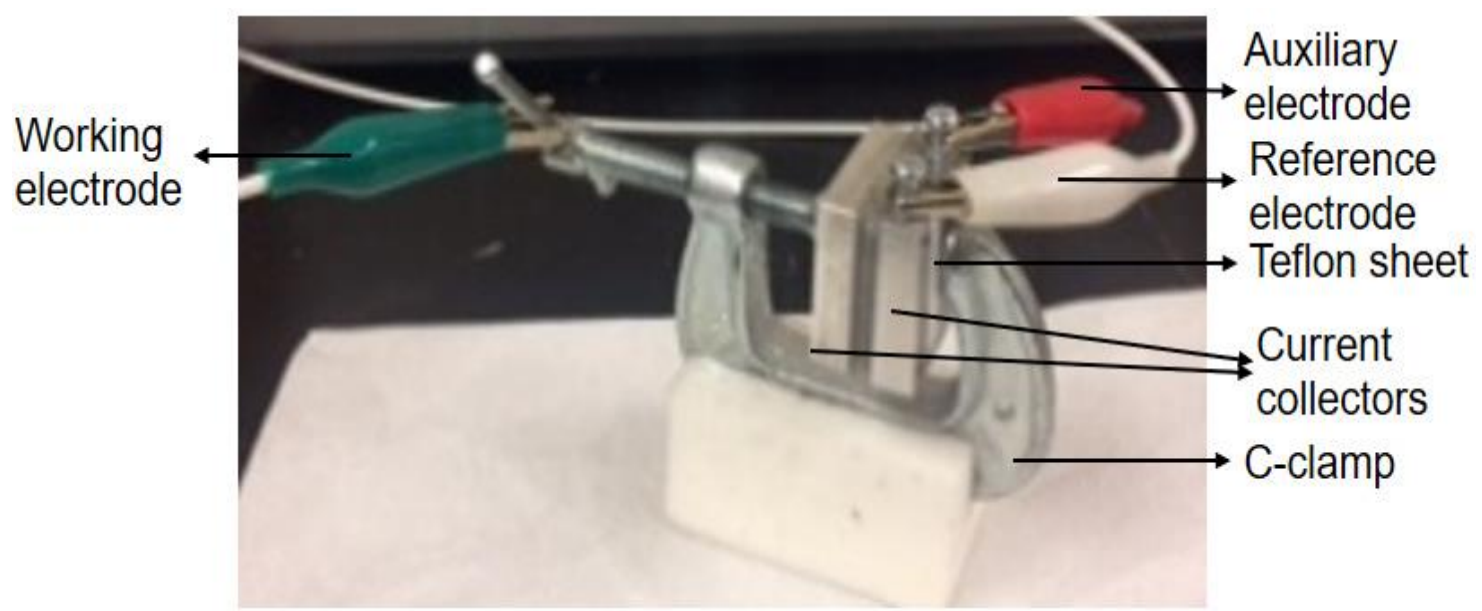

Figure 6.5 Configuration of the prototype cell in sandwich configuration.

\subsection{Performance Analysis}

\subsubsection{Cyclic Voltammetry Analysis}

CVs were recorded using $\mathrm{CH} 660$ Electrochemical Analyzer/Workstation and the potential of the working electrode was swept between 0 to $2 \mathrm{~V}$ at scan rates of (a) $0.005 \mathrm{~V} / \mathrm{s}$, (b) $0.01 \mathrm{~V} / \mathrm{s}$, and (c) $0.1 \mathrm{~V} / \mathrm{s}$ as shown in Figure 6.6. In all the cyclic voltammograms, similar responses were obtained. In the forward scan, anodic current was observed due to the oxidation of ferrocene dicarboxylic acid. Whereas, during the reverse scan, no cathodic peak current was seen suggesting leakage through separator membranes between the oxidation and reduction half-cells. 

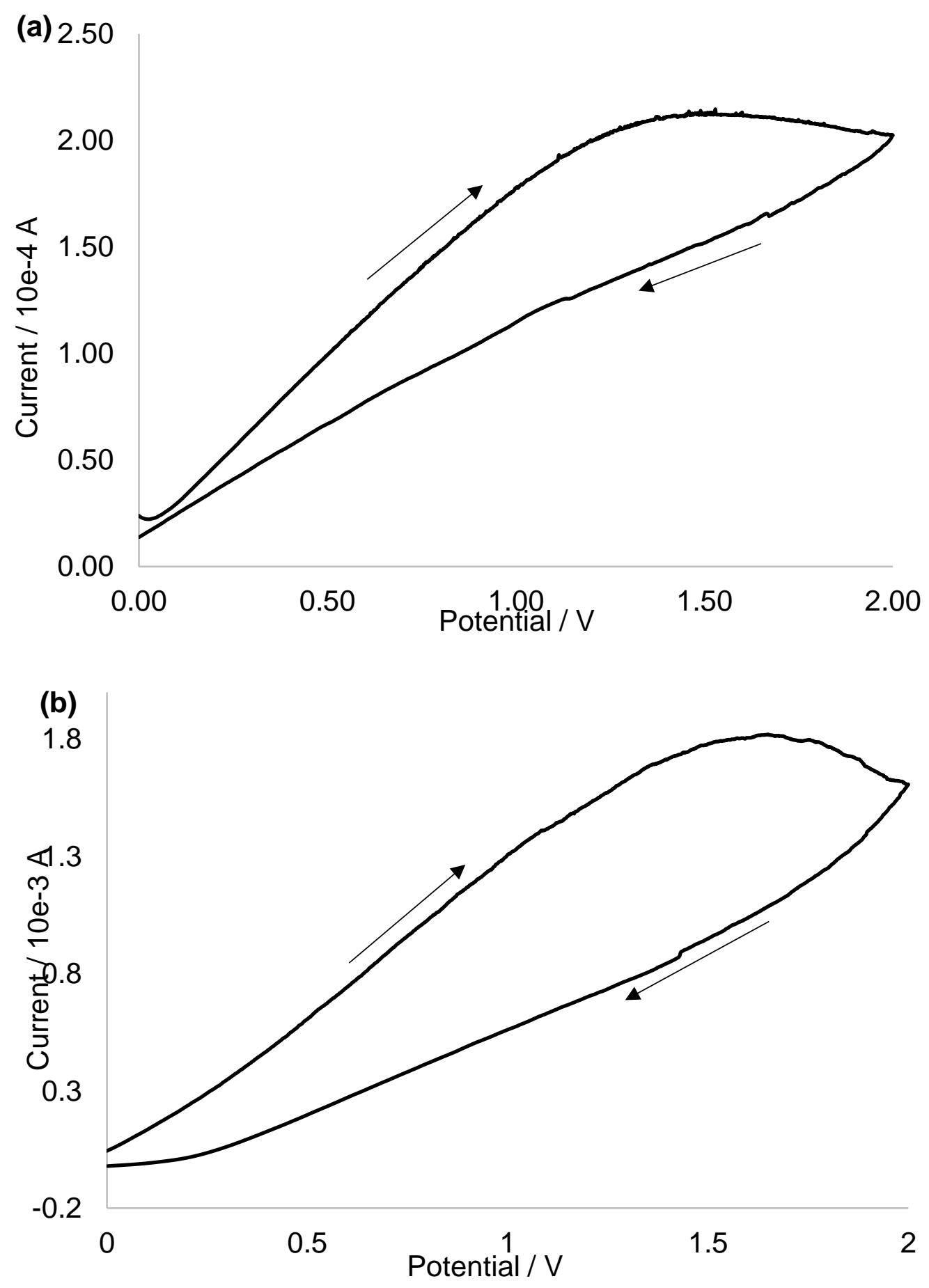


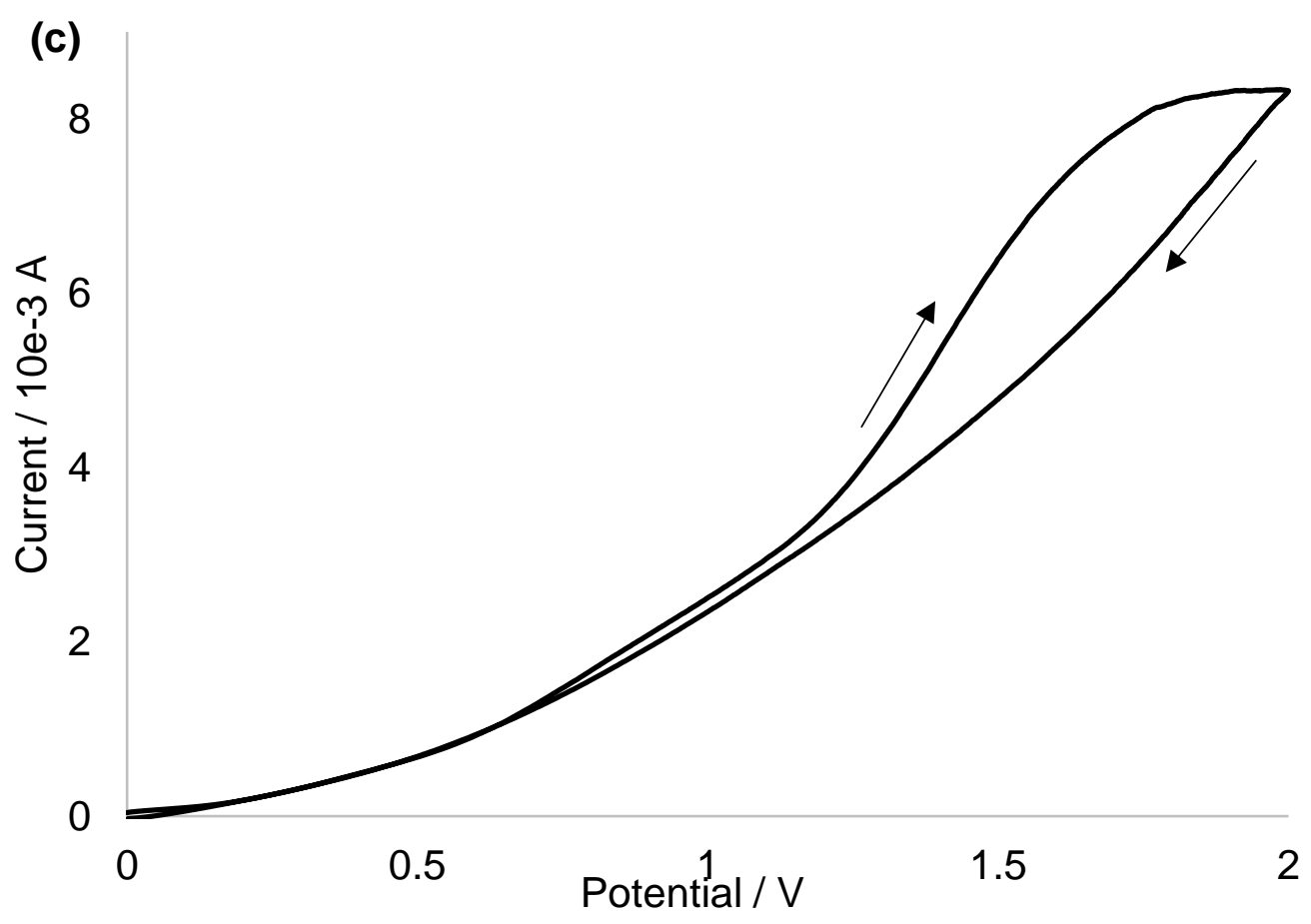

Figure 6.6 Cyclic Voltammograms of the prototype cell at scan rates (a) 0.005 $\mathrm{V} / \mathrm{s}$, (b) $0.01 \mathrm{~V} / \mathrm{s}$, and (c) $0.1 \mathrm{~V} / \mathrm{s}$.

\subsubsection{Chronoamperometry Analysis}

To investigate the charging and discharging currents of the prototype cell, chronoamperometry (CA) was done using Princeton Applied Research / EG\&G 263A Potentiostat. Before recording CA, the cell was equilibrated for $500 \mathrm{~s}$ at 0 V. Once the current reached zero, the applied potential was stepped from 0 to 1 $\mathrm{V}$ and the cell was held for $500 \mathrm{~s}$ to record response. Then the applied potential was stepped down from 1 to $0 \mathrm{~V}$ for $500 \mathrm{~s}$. Similar double pulse CA experiments were performed for $0.5 \mathrm{~s}, 5 \mathrm{~s}$ and $50 \mathrm{~s}$ in the same potential range. The resulting CA graphs are shown in Figure 6.7 (a) $0.5 \mathrm{~s}$, (b) $5 \mathrm{~s}$, (c) $50 \mathrm{~s}$, and (d) $500 \mathrm{~s}$. 

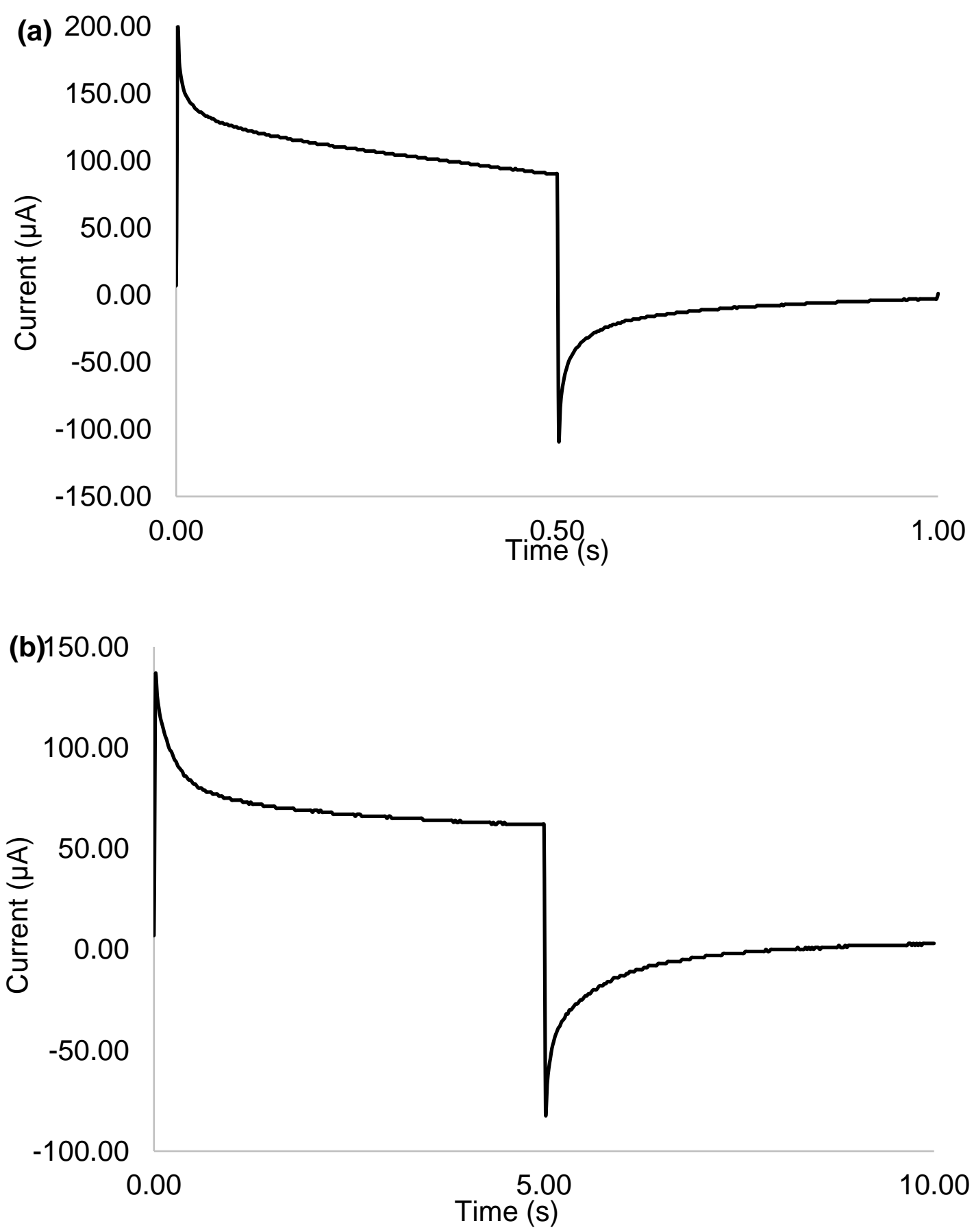

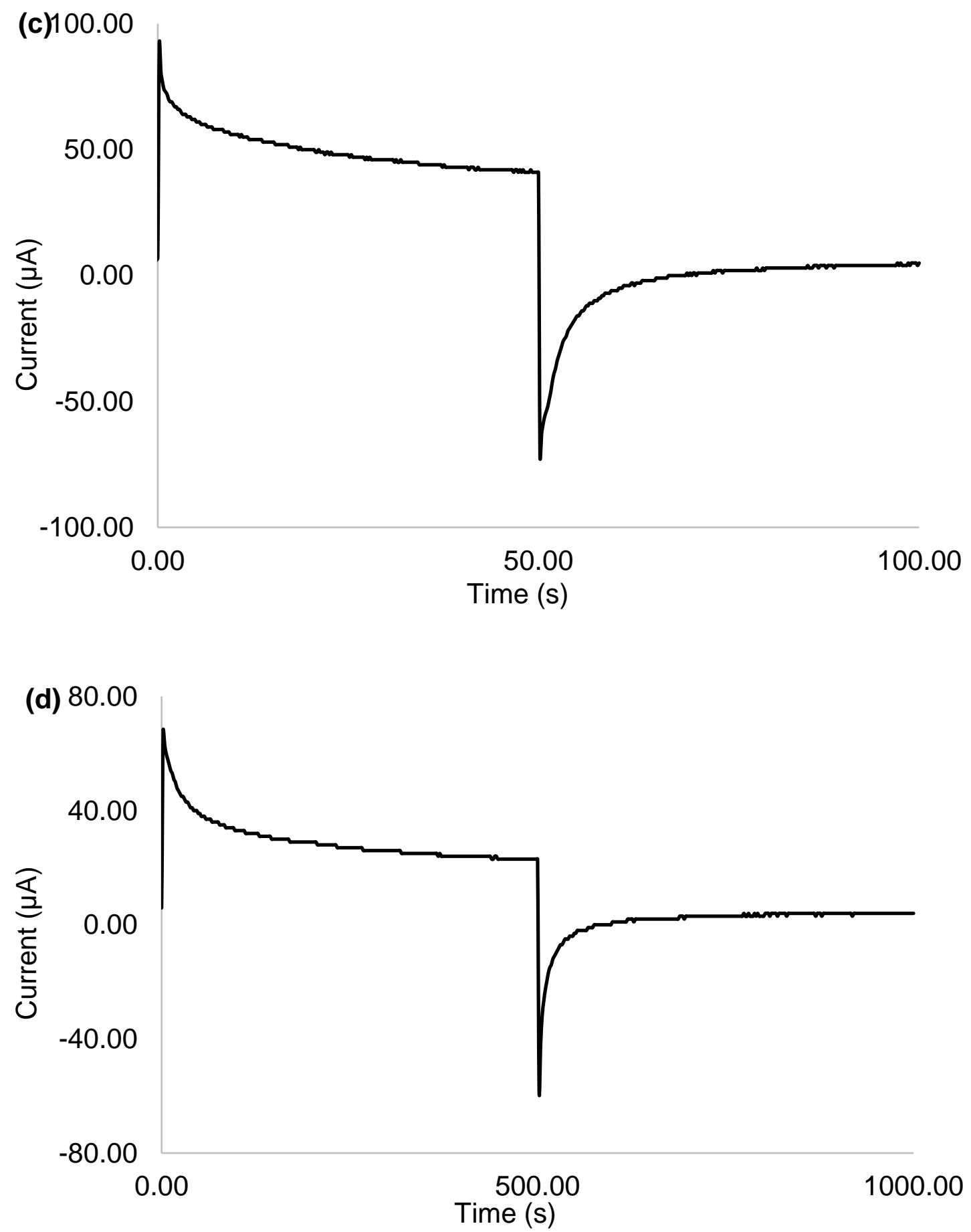

Figure 6.7 Chronoamperometry graphs of the prototype cell at (a) $0.5 \mathrm{~s}$, (b) $5 \mathrm{~s}$, (c) $50 \mathrm{~s}$, and (d) $500 \mathrm{~s}$. 
In all the CA graphs, the current decreased gradually with increasing time intervals from $0.5 \mathrm{~s}$ to $500 \mathrm{~s}$. At longer time scales ( $50 \mathrm{~s}, 500 \mathrm{~s}$ ) the battery showed less relative capacitive current as compared to the shorter time scales $(0.5 \mathrm{~s}, 5 \mathrm{~s})$. This is because the capacitive current decays exponentially with time, as compared to faradaic current, according to Equation 6.6. Therefore, at larger time scales (500 s), mostly faradaic current is observed. Unfortunately, charging and discharging currents in all the CA graphs were unequal, as shown in Table 6.1. This unequal current means that the current is not being stored fully. One possibility is that there is a leakage of current, possibly due to penetration of SWCNTs through the separator membrane.

Table 6.1 Charging and discharging currents.

\begin{tabular}{|l|l|l|}
\hline Time $(\mathrm{s})$ & Charging Current $\left(10^{-4} \mathrm{~A}\right)$ & Discharging Current $\left(10^{-4} \mathrm{~A}\right)$ \\
\hline 0.5 & 1.97 & -1.07 \\
\hline 5 & 1.36 & -0.81 \\
\hline 50 & 0.92 & -0.72 \\
\hline 500 & 0.68 & -0.58 \\
\hline
\end{tabular}

\subsection{Conclusions and Future Work}

In this work, the battery material, a SWCNT-Fc polymer composite was successfully synthesized. This material showed an excellent redox behavior and electrochemical properties and an appropriate material for a reduction half-cell in a prototype cell. The material for the oxidation half-cell was a SWCNT-viologen (V) polymer composite which showed promising electrochemical behavior and was synthesized by my laboratory colleague Kanishka Rana. ${ }^{27}$ SWCNT-Fc polymer and SWCNT-V polymer composites were used in the fabrication of the 
prototype cell. The CV and CA results for the hybrid cell were not conclusive in showing the desired charging and discharging curves. The CV responses exhibited an anodic current in the forward scan whereas no cathodic current plateau was seen in the reverse scan. Similarly, CA graphs showed charging current, but the discharging current was much lower than that. Both CV and CA responses suggested the leakage of current and short circuiting of the SWCNTpolymer composites through the separator membrane.

The battery materials used in the prototype cell showed promising electrochemical responses. But the charging-discharging behavior of the hybrid prototype cell is yet to be fully understood. Further study could focus on using the different types of separator membranes such as nylon membranes of smaller pore size to avoid leakage of carbon nanotubes and short circuiting of the cell. Another type of gasket could be used instead of silicone as it swells by absorbing acetonitrile leading to low conductivity and diffusivity of electrolyte ions in the electrode. A non-volatile alternative solvent could be used because acetonitrile being volatile might be drying up the sample, resulting in low diffusivity and mobility of electrolyte ions through the separator. 


\section{REFERENCES}

(1) Higgins, D.; Zamani, P.; Yu, A.; Chen, Z. The Application of Graphene and Its Composites in Oxygen Reduction Electrocatalysis: A Perspective and Review of Recent Progress. Energy Environ. Sci. 2016, 9, 357-390.

(2) Burke, A. Ultracapacitors: Why, How, and Where is the Technology. J. Power Sources 2000, 91 (1), 37-50.

(3) Miller, J. R.; Simon, P.; Patrice, S. Electrochemical Capacitors for Energy Management. Sci. Mag. 2008, 321 (5889), 651-652.

(4) Abuadmah, $\mathrm{H}$. Fuel Cells Versus Heat Engines: A Perspective of Thermodynamic and Production Efficiencies. 2012.

(5) Kunze, J.; Stimming, U. Electrochemical versus Heat-Engine Energy Technology: A Tribute to Wilhelm Ostwald's Visionary Statements. Angew. Chemie - Int. Ed. 2009, 48 (49), 9230-9237.

(6) Gonzalez, A.; Goikolea, E.; Barrena, J. A.; Mysyk, R. Review on Supercapacitors: Technologies and Materials. Renew. Sustain. Energy Rev. 2016, 58, 1189-1206.

(7) Conway, B. E. Electrochemical Supercapacitors: Scientific Fundamentals and Technological Applications; Kluwer Academic/ Plenum Publisher: New York: Ontario, 1999; pp 11-31.

(8) Conway, B. E. Transition from "Supercapacitor" to "Battery" Behavior in Electrochemical Energy Storage. J. Electrochem. Soc. 1991, 138, 15391548.

(9) Winter, M.; Brodd, R. J. What Are Batteries, Fuel Cells, and Supercapacitors? Chem. Rev. 2004, 104 (10), 4245-4269.

(10) Tom Ding; Mary Deng. http://www.limn2o4.com/ (accessed Jan 1, 2017).

(11) Vinodkumar Etacheri, Rotem Marom, Ran Elazari, G. S. and D. A. Challenges in the Development of Advanced Li-Ion Batteries: A Review. Energy Environ. Sci. 2011, 4, 3243-3262. 
(12) Liu, Y.; Yu, F.; Wang, X. W.; Wen, Z. B.; Zhu, Y. S.; Wu, Y. P. Nanostructured Oxides as Cathode Materials for Supercapacitors. In Nanomaterials in Advanced Batteries and Supercapacitors; Lockwood, D. J.; Ozoemena, K. I.; Chen, S; Nanostructure Science and Technology; Springer International: Switzerland, 2016; pp 205-209.

(13) Kotz, R.; Carlen, M.; Kötz, R.; Carlen, M.; Kötz, R.; Carlen, M.; Carlen, M. Principles and Applications of Electrochemical Capacitors. Electrochim. Acta 2000, 45 (15-16), 2483-2498.

(14) Obreja, V. V. N. On the Performance of Supercapacitors with Electrodes Based on Carbon Nanotubes and Carbon Activated Material-A Review. Phys. E Low-Dimensional Syst. Nanostructures 2008, 40 (7), 2596-2605.

(15) Shah, V.; Joshi, J. A.; Maheshwari, R.; Roy, R. Review of Ultracapacitor Technology and Its Applications. Fifteenth Natl. Power Syst. Conf. (NPSC), IIT Bombay 2008, 142-147.

(16) Namisnyk, A. M. A Survey of Electrochemical Supercapacitor Technology. University of Technology, Sydney. June 2003.

(17) Halper, M.; Ellenbogen, J. Supercapacitors: A Brief Overview. Rep. No. MP 05W0000272. 2006, 1-29.

(18) Simon, P.; Gogotsi, Y. Materials for Electrochemical Capacitors. Nat. Mater. 2008, 7, 845-854.

(19) Supercapacitor. https://en.wikipedia.org/wiki/Supercapacitor (accessed Jan 1, 2017).

(20) Zhao, X.; Sánchez, B. M.; Dobson, P. J.; Grant, P. S. The Role of Nanomaterials in Redox-Based Supercapacitors for next Generation Energy Storage Devices. Nanoscale 2011, 3 (3), 839-855.

(21) Bockris, J. O.; Devanathan, M. A. V.; Müller, K. On the Structure of Charged Interfaces. Proc. R. Soc. A Math. Phys. Eng. Sci. 1963, 274 (1356), 55-79.

(22) Wang, H.; Pilon, L. Physical Interpretation of Cyclic Voltammetry for Measuring Electric Double Layer Capacitances. Electrochim. Acta 2012, $64,130-139$. 
(23) Zhang, L. L.; Zhou, R.; Zhao, X. S. Carbon-Based Materials as Supercapacitor Electrodes. J. Mater. Chem. 2009, 38 (29), 2520-2531.

(24) Simon, P.; Gogotsi, Y. Capacitive Energy Storage in Nanostructured CarbonElectrolyte Systems. Acc. Chem. Res. 2013, 46, 1094-1103.

(25) Wang, G.; Zhang, L.; Zhang, J. A Review of Electrode Materials for Electrochemical Supercapacitors. Chem. Soc. Rev. 2012, 41 (41), 797828.

(26) Zhong, C.; Deng, Y.; Hu, W.; Qiao, J.; Zhang, L.; Zhang, J. A Review of Electrolyte Materials and Compositions for Electrochemical

Supercapacitors. Chem. Soc. Rev. 2015, 44 (21), 7484-7539.

(27) Rana, K. Preparation and Characterization of a Viologen Containing Main Chain PEG-Carbon Nanotubes Phase for Hybrid Supercapacitor Application. M.S. Thesis, San Jose State University, San Jose, CA, 2017.

(28) Largeot, C.; Portet, C.; Chmiola, J.; Taberna, P. L.; Gogotsi, Y.; Simon, P. Relation between the Ion Size and Pore Size for an Electric Double-Layer Capacitor. J. Am. Chem. Soc. 2008, 130 (9), 2730-2731.

(29) Wang Odom, T.; Huang, J.-L.; Lieber, C. M. Single-Walled Carbon Nanotubes From Fundamental Studies to New Device Concepts. Ann. N.Y. Acad. Sci 2002, 960, 203-215.

(30) Sarangdevot, K.; Sonigara, B. S. The Wondrous World of Carbon Nanotubes: Structure, Synthesis, Properties and Applications. J. Chem. Pharm. Res. 2015, 7 (6), 916-933.

(31) Frackowiak, E.; Béguin, F. Carbon Materials for the Electrochemical Storage of Energy in Capacitors. Carbon N. Y. 2001, 39 (6), 937-950.

(32) Stephan, A. M. Review on Gel Polymer Electrolytes for Lithium Batteries. Eur. Polym. J. 2006, 42 (1), 21-42.

(33) Senthilkumar, S. T.; Selvan, R. K.; Melo, J. S. Redox Additive/Active Electrolytes: A Novel Approach to Enhance the Performance of Supercapacitors. J. Mater. Chem. A 2013, 1 (40), 12386-12394. 
(34) Brian, P. A.; Zhou, X.-F.; Richard, G. Electrochemical Sensing Using an Enzyme Electrode, 2006.

(35) Sharma, P.; Bhatti, T. S. A Review on Electrochemical Double-Layer Capacitors. Energy Convers. Manag. 2010, 51 (12), 2901-2912.

(36) Saleh Ahammad, A. J.; Lee, J.; Rahman, M. Electrochemical Sensors Based on Carbon Nanotubes. Sensors 2009, 9, 2289-2319.

(37) Mittal, G.; Dhand, V.; Rhee, K. Y.; Park, S.-J.; Lee, W. R. A Review on Carbon Nanotubes and Graphene as Fillers in Reinforced Polymer Nanocomposites. J. Ind. Eng. Chem. 2015, 21, 11-25.

(38) Tasis, D.; Tagmatarchis, N.; Bianco, A.; Prato, M. Chemistry of Carbon Nanotubes. Chem. Rev. 2006, 106 (3), 1105-1136.

(39) Karousis, N.; Tagmatarchis, N.; Tasis, D. Current Progress on the Chemical Modification of Carbon Nanotubes. Chem. Rev. 2010, 110 (9), 5366-5397.

(40) Hu, H.; Zhao, B.; Hamon, M. A.; Kamaras, K.; Itkis, M. E.; Haddon, R. C. Sidewall Functionalization of Single-Walled Carbon Nanotubes by Addition of Dichlorocarbene. J. Am. Chem. Soc. 2003, 125 (48), 14893-14900.

(41) Zhang, J.; Zou, H.; Qing, Q.; Yang, Y.; Li, Q.; Liu, Z. Effect of Chemical Oxidation on the Structure of Single-Walled Carbon Nanotubes. J. Phys. Chem. B 2003, 3712-3718.

(42) Blanco, M.; Álvarez, P.; Blanco, C.; Jiménez, M. V.; Pérez-Torrente, J. J.; Oro, L. A.; Blasco, J.; Cuartero, V.; Menéndez, R. Enhancing the Hydrogen Transfer Catalytic Activity of Hybrid Carbon Nanotube-Based NHC-iridium Catalysts by Increasing the Oxidation Degree of the Nanosupport. Catal. Sci. Technol. 2016, 6 (14), 5504-5514.

(43) Najafi, E.; Kim, J. Y.; Han, S. H.; Shin, K. UV-Ozone Treatment of MultiWalled Carbon Nanotubes for Enhanced Organic Solvent Dispersion. Colloids Surfaces A Physicochem. Eng. Asp. 2006, 284-285, 373-378.

(44) Colthup B, N.; Daly, L. H.; Wiberley, S. E. Introduction to Infrared and Raman Spectroscopy; Academic Press Inc. (London) Ltd., 1975. 
(45) Thomsen, C.; Reich, S. Raman Scattering in Carbon Nanotubes. Carbon Nanotub. 2007, 232, 115-234.

(46) Belin, T.; Epron, F. Characterization Methods of Carbon Nanotubes: A Review. Mater. Sci. Eng. B 2005, 119 (2), 105-118.

(47) Pike. Application Note - 0402 ATR Theory and Applications. Pike Technol. 1-3.

(48) PerkinEImer. FT-IR Spectroscopy Attenuated Total Reflectance (ATR). PerkinElmer Life Anal. Sci. 2005, 1-5.

(49) Wepasnick, K. A.; Smith, B. A.; Bitter, J. L.; Howard Fairbrother, D. Chemical and Structural Characterization of Carbon Nanotube Surfaces. Anal. Bioanal. Chem. 2010, 396 (3), 1003-1014.

(50) Muench, S.; Wild, A.; Friebe, C.; Haupler, B.; Janoschka, T.; Schubert, U. S. Polymer-Based Organic Batteries. Chem. Rev. 2016, 116 (16), 94389484.

(51) Xiang, J.; Burges, R.; Häupler, B.; Wild, A.; Schubert, U. S.; Ho, C.-L.; Wong, W.-Y. Synthesis, Characterization and Charge-Discharge Studies of Ferrocene-Containing Poly(fluorenylethynylene) Derivatives as Organic Cathode Materials. Polymers 2015, 68, 328-334.

(52) Steglish Esterification. http://www.organic-chemistry.org (accessed Sep 4, 2017)

(53) Neises, B.; Steglich, W. Simple Method for the Esterification of Carboxylic Acids. Angew. Chemie Int. Ed. 1978, 17 (7), 522-524.

(54) Nakahata, M.; Takashima, Y.; Yamaguchi, H.; Harada, A. RedoxResponsive Self-Healing Materials Formed from Host-guest Polymers. Nat. Commun. 2011, 2, 511.

(55) Wang, Y.; Wang, H.; Chen, Y.; Liu, X.; Jin, Q.; Ji, J. PH and Hydrogen Peroxide Dual Responsive Supramolecular Prodrug System for Controlled Release of Bioactive Molecules. Colloids Surf. B Biointerfaces 2014, 121, 189-195. 
(56) Tian, J.; Chen, J.; Ge, C.; Liu, X.; He, J.; Ni, P.; Pan, Y. Synthesis of PEGylated Ferrocene Nanoconjugates as the Radiosensitizer of Cancer Cells. Bioconjug. Chem. 2016, 27 (6), 1518-1524.

(57) Proton Nuclear Magnetic Resonance Spectroscopy (H-Nmr). http://www.chem.ucla.edu (accessed Sep 1, 2017).

(58) Keeler, J. H. Understanding NMR Spectroscopy; Wiley, 2002.

(59) Breitmaier, E.; Voelter, W. Carbon-13 NMR Spectroscopy; VCH Publishers Inc.,Deerfield Bch, FL, 1987.

(60) Kissinger, P. T.; Heineman, W. R. Cyclic Voltammetry. J. Chem. Educ. 1983, 60 (9), 702.

(61) Vanýsek, P.; Tavassol, H.; Pilson, K.-L. The Role of Reference and Counter Electrodes in Electrochemical Impedance Measurement. Electrochem. Soc. 2013.

(62) Stulík, K.; Amatore, C.; Holub, K.; Marecek, V.; Kutner, W. Microelectrodes. Definitions, Characterization, and Applications (Technical Report). Pure Appl. Chem. 2000, 72 (8), 1483-1492.

(63) Ching, S.; Dudek, R.; Tabet, E. Cyclic Voltammetry with Ultramicroelectrodes. J. Chem. Educ. 1994, 71 (7), 602-605.

(64) Bolinger, R. W. The Deployment of Digital Simulation Tools to Verify Cyclic Voltammetry Experiments, Ph.D. Thesis, Swiss Federal Institute of Technology Zurich, 2000.

(65) Jung, D. H.; Ko, Y. K.; Jung, H. T. Aggregation Behavior of Chemically Attached Poly(ethylene Glycol) to Single-Walled Carbon Nanotubes (SWNTs) Ropes. Mater. Sci. Eng. C 2004, 24 (1-2), 117-121.

(66) Zhao, B.; Zhao, B.; Hu, H.; Hu, H.; Yu, A.; Yu, A.; Perea, D.; Perea, D.; Haddon, R. C.; Haddon, R. C. Synthesis and Characterization of Water Soluble Single-Walled Carbon Nanotube Graft Copolymers. Science. 2005, 37, 8197-8203. 
(67) Zhao, B.; Hu, H.; Haddon, R. C. Synthesis and Properties of a WaterSoluble Single-Walled Carbon Nanotube-poly (m-Aminobenzene Sulfonic Acid) Graft Copolymer. Adv. Funct. Mater. 2004, 14 (1), 71-76.

(68) Hyk, W.; Nowicka, A.; Stojek, Z. Direct Determination of Diffusion Coefficients of Substrate and Product by Chronoamperometric Techniques at Microelectrodes for Any Level of lonic Support. Anal. Chem. 2002, 74 (1), 149-157.

(69) Avci, E. An Electrochemical Study of the Deposition of Copper and Silver on Thymine Modified Au(111). Exp. methods 2007, 28-42. 